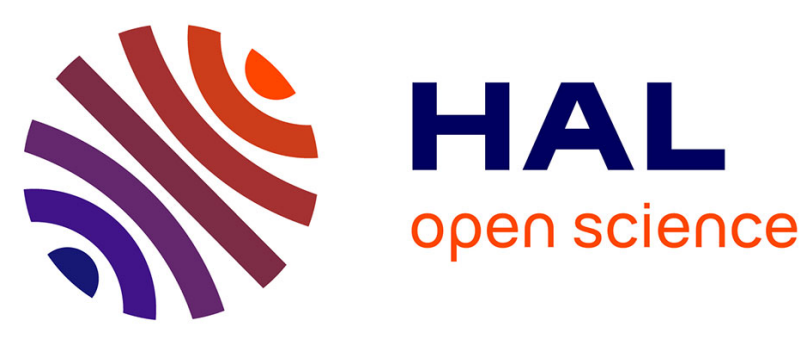

\title{
Koiter Estimate Revisited
}

Monique Dauge, Erwan Faou

\section{To cite this version:}

Monique Dauge, Erwan Faou. Koiter Estimate Revisited. Mathematical Models and Methods in Applied Sciences, 2010, 20 (1), pp. 1-42. 10.1142/S0218202510004131 . hal-00202628v2

\section{HAL Id: hal-00202628 \\ https://hal.science/hal-00202628v2}

Submitted on 25 Mar 2009

HAL is a multi-disciplinary open access archive for the deposit and dissemination of scientific research documents, whether they are published or not. The documents may come from teaching and research institutions in France or abroad, or from public or private research centers.
L'archive ouverte pluridisciplinaire HAL, est destinée au dépôt et à la diffusion de documents scientifiques de niveau recherche, publiés ou non, émanant des établissements d'enseignement et de recherche français ou étrangers, des laboratoires publics ou privés. 


\title{
Koiter Estimate Revisited
}

\author{
Monique Dauge AND ERWAN FAOU
}

\begin{abstract}
We prove a general adimensional energy estimate between the solution of the three-dimensional Lamé system on a thin clamped shell and a displacement reconstructed from the solution of the classical two-dimensional Koiter model. This estimate only involves the thickness parameter $\varepsilon$, constants attached to the mid-surface $S$, the two-dimensional energy of the solution of the Koiter model and "wave-lengths" associated with this latter solution. This bound is in the same spirit as Koiter's heuristic estimate in [26] and can be viewed as an a posteriori estimation of the modeling error by means of the two-dimensional solution. It is general with respect to the geometry of the mid-surface $S$ which is an arbitrary smooth manifold with boundary. Taking boundary layer terms into account, we prove that our estimates are sharp in the cases of plates and elliptic shells.
\end{abstract}

\section{INTRODUCTION}

This paper deals with shell theory whose aim is the approximation of the three-dimensional linear elastic shell problem by a two-dimensional problem posed on the mid-surface. This is an old and difficult question. As written by KOITER \& SIMMONDS in 1972 [28] "Shell theory attempts the impossible: to provide a two-dimensional representation of an intrinsically three-dimensional phenomenon."

\section{A FRAMEWORK}

A shell is a thin three-dimensional domain characterized by its mid-surface $S$ and its (half)thickness $\varepsilon$. Such a domain, denoted by $\Omega^{\varepsilon}$, coincides with the surface $S$ thickened in its normal direction by the length $\varepsilon$ which is assumed to be small in comparison with the characteristic lengths of $S$. For the body represented by $\Omega^{\varepsilon}$, the simplest and most classical assumption is to consider homogeneous and isotropic material laws.

For a given load $\boldsymbol{f}$, let $\boldsymbol{u}$ be the displacement field, solution of the problem $\left(P_{3 \mathrm{D}}\right)$ consisting of the three-dimensional Lamé system on $\Omega^{\varepsilon}$ with clamped boundary conditions on its lateral boundary. We consider this $\boldsymbol{u}$ as the "exact" solution and address the question of the approximation of $\boldsymbol{u}$ via the solution $\boldsymbol{z}$ of a problem $\left(P_{2 \mathrm{D}}\right)$ posed on the mid-surface $S$.

Many papers deal with this question. Concerning the classical aspects of the derivation of shell models, let us quote KoITER [25, 26, 27], John [23], NAGHDi [32], NovozhiLOV [35]. Concerning plates (that is, flat shells), the derivation of the first two-dimensional model was done much earlier, see KIRCHHOFF [24]. 
Most of classical shell models rely on a $3 \times 3$ system of equations on $S$ depending on $\varepsilon$, which can be written in the form

$$
\mathrm{K}(\varepsilon):=\mathrm{M}+\varepsilon^{2} \mathrm{~B}
$$

where $\mathrm{M}$ is the membrane operator on $S$ and $\mathrm{B}$ a bending operator. The above authors all agree about the definition of the membrane operator $M$. On the contrary, different expressions for B can be found in the literature. The most natural in a geometrical and mechanical point of view, is the one given by KOITER (see [26]) but the question of determining the best model was very controversial (see in particular [8] and the discussion in [27, 32]). Without special mention, we always take $\mathrm{K}(\varepsilon)$ as the Koiter operator.

So the equation in the mid-surface $S$ takes the form $\mathrm{K}(\varepsilon) \boldsymbol{z}=\boldsymbol{g}$, with the mean value $\boldsymbol{g}$ of the load $\boldsymbol{f}$ across each normal fiber to $S$. When considering laterally clamped shells, this equation has to be complemented by the Dirichlet boundary condition and defines problem $\left(P_{2 \mathrm{D}}\right)$. The unique solvability of this problem was proved by BERNADOU \& CIARLET [6]. The question of the "validity" of $\boldsymbol{z}$ solution of problem $\left(P_{2 \mathrm{D}}\right)$ is central in shell theory. However, it has been proved that in general, $\boldsymbol{z}$ is not an approximation of $\boldsymbol{u}$ in energy norm, but in weaker norms, as stated and proved by SANCHEZ-PALENCIA [37] and CiARLET, Lods, MiaRA $[10,12,11]$. Considering the energy norm, the approximation of $\boldsymbol{u}$ by $\boldsymbol{z}$ relies on a reconstruction operator mapping the two-dimensional displacement $z$ onto a three-dimensional displacement $\mathrm{U} z$.

\section{B KOITER ESTIMATE AND THIN PLATE COUNTER-EXAMPLE}

KOITER proposed for $\mathrm{U} z$ a modified Kirchhoff-Love three-dimensional displacement, which we may write as

$$
\mathrm{U} z:=\mathrm{U}^{\mathrm{KL}} \boldsymbol{z}+\mathrm{U}^{\mathrm{cmp}} \boldsymbol{z}
$$

where $\mathrm{U}^{\mathrm{KL}} \boldsymbol{z}$ is the Kirchhoff-Love displacement associated with $z$ and the complementary term $U^{\mathrm{cmp}} \boldsymbol{z}$ is a transverse displacement quadratic in the normal variable $x_{3}$ : see (2.23) below for their expressions. In his main papers [26, 27], KOITER obtained for "standard" load $\boldsymbol{f} \equiv \boldsymbol{g}$ independent of $x_{3}$ the following tentative energy estimate:

$$
\mathrm{E}_{3 \mathrm{D}}^{\varepsilon}[\boldsymbol{u}-\mathrm{U} \boldsymbol{z}] \leq C_{S}\left(\frac{\varepsilon}{R}+\frac{\varepsilon^{2}}{L^{2}}\right) \mathrm{E}_{2 \mathrm{D}}^{\varepsilon}[\boldsymbol{z}]
$$

where $\mathrm{E}_{3 \mathrm{D}}^{\varepsilon}$ is the quadratic energy functional associated with problem $\left(P_{3 \mathrm{D}}\right)$ and $\mathrm{E}_{2 \mathrm{D}}^{\varepsilon}$ is the quadratic "physical" energy associated with problem $\left(P_{2 \mathrm{D}}\right)$. Moreover $1 / R$ denotes the maximum principal curvature of $S$ and $L$ a "wave length" associated with the solution $z$ and which depends on $\varepsilon$ in general.

But, in the situation of plates, $L$ does not depend on $\varepsilon$ and, of course, $\frac{1}{R}=0$. Two years after the publication of [26, 27], it was already known that estimate (1.3) does not hold as $\varepsilon \rightarrow 0$ for plates. We read in [28] "The somewhat depressing conclusion for most shell problems is, similar to the earlier conclusions of GOL'DENWEIZER, that no better accuracy of the solutions can be expected than of order $\frac{\varepsilon}{L}+\frac{\varepsilon}{R}$, even if the equations of first-approximation shell theory would permit, in principle, an accuracy of order $\frac{\varepsilon^{2}}{L^{2}}+\frac{\varepsilon}{R}$." 
The reason for this is also explained by JOHN [23] in these terms "Concentrating on the interior we sidestep all kinds of delicate questions, with an attendant gain in certainty and generality. The information about the interior behavior can be obtained much more cheaply (in the mathematical sense) than that required for the discussion of boundary value problems, which form a more "transcendental" stage.".

The presence of boundary layer terms for thin plates in the vicinity of the lateral part of the boundary was already pointed out by GOL'DENWEIZER [21] but a multi-scale asymptotic expansion combining (for plates) inner (boundary layer) and outer (regular) parts was only available later, see Chapters 15 and 16 in [31] and its bibliographical comments. A more specific form adapted for clamped thin plates is provided by NAZAROV \& ZORIN in [33] and DAUGE \& GRUAIS in [15]. From these results we can deduce the sharp estimates for plates, valid for a "standard" load, see $[16, \S 12]$

$$
\mathrm{E}_{3 \mathrm{D}}^{\varepsilon}\left[\boldsymbol{u}^{\varepsilon}-\mathrm{U} \boldsymbol{z}^{\varepsilon}\right] \leq b_{S} \varepsilon \mathrm{E}_{2 \mathrm{D}}^{\varepsilon}\left[\boldsymbol{z}^{\varepsilon}\right], \quad \text { as } \quad \varepsilon \rightarrow 0 .
$$

In (1.4), the factor $\varepsilon$ in the bound is sharp and comes from the contribution of the three-

dimensional boundary layer term along the lateral part of the boundary, and $b_{S}^{-1}$ has the dimension of a length - so that $b_{S} \varepsilon$ is adimensional. This contradicts estimate (1.3), since in this case it would yield $\varepsilon^{2}$ instead of $\varepsilon$.

\section{C RigOROUS ESTIMATES}

For shells, the complexity of a multi-scale analysis (if possible) is much higher than for plates. There is at least one situation where such an analysis was successfully performed: the case of clamped elliptic shells. In [19, 20], FAOU proved that

1. The solution $\boldsymbol{z}=\boldsymbol{z}^{\varepsilon}$ of the Koiter problem $\left(P_{2 \mathrm{D}}\right)$ has a boundary layer in the vicinity of $\partial S$ at a scale $\sqrt{\varepsilon}$, which yields that the wave length $L$ is a $\mathcal{O}(\sqrt{\varepsilon})$,

2. The solution $\boldsymbol{u}=\boldsymbol{u}^{\varepsilon}$ of the Lamé problem $\left(P_{3 \mathrm{D}}\right)$ has a complete three-scale asymptotics combining regular and boundary layer terms at scales $\sqrt{\varepsilon}$ and $\varepsilon$.

Relying on this one can prove that estimate (1.4) holds true, and that it is sharp. But now, both terms in the sum $\frac{\varepsilon^{2}}{L^{2}}+\frac{\varepsilon}{R}$ are a $\mathcal{O}(\varepsilon)$ and this proves that the first Koiter estimate (1.3) is asymptotically valid for clamped elliptic shells.

In a fairly general situation for the geometry of the mid-surface, LODS \& MARDARE proved in $[29,30]$ the estimate

$$
\mathrm{E}_{3 \mathrm{D}}^{\varepsilon}\left[\boldsymbol{u}^{\varepsilon}-\mathrm{U} \boldsymbol{z}^{\varepsilon}\right] \leq C \varepsilon^{1 / 2} \mathrm{E}_{3 \mathrm{D}}^{\varepsilon}\left[\boldsymbol{u}^{\varepsilon}\right]
$$

Here the constant $C$ depends on the load $f$. This strong result shows the convergence of $U \boldsymbol{z}^{\varepsilon}$ towards $\boldsymbol{u}^{\varepsilon}$ in energy norm. However it does not validate or invalidate Koiter type estimate. 


\section{D OUTLINE OF RESULTS}

In this paper, we prove general estimates in the spirit of (1.3) without a priori knowledge of multi-scale expansions for $\boldsymbol{u}$ and $\boldsymbol{z}$. The complete result is given in Theorem 2.8. Our estimate now involves the three following constants:

a) A global wave length $L$ associated with $z$ similar to the one which Koiter used,

b) A lateral wave length $\ell$ for $\boldsymbol{z}$, allowing to take boundary layer effects into account,

c) A curvature constant $r$ depending on the curvature of $S$ and its derivatives.

Besides these three main quantities, two more lengths D and $d$ attached to the mid-surface $S$ take part in our statement.

Let us briefly describe our result under a simplifying hypothesis. The constant $L$ typically describes the characteristic length of layers appearing in the shell. According to the formal result in [36], this wave length can typically be assumed of size $L \geq \sqrt{L_{S} \varepsilon}$ where $L_{S}$ has the dimension of a length and is uniformly bounded in $\varepsilon$. Under this assumption, and in the specific case where the loading forces $f$ are constant along each normal fiber of the shell, our general estimate (2.24) in Theorem 2.8 yields

$$
\mathrm{E}_{3 \mathrm{D}}^{\varepsilon}[\boldsymbol{u}-\mathrm{U} \boldsymbol{z}] \leq a_{S}\left(\frac{\varepsilon}{\ell}+\frac{\varepsilon^{2}}{\mathrm{r}^{2}}+\frac{\varepsilon^{2}}{L^{2}}\right) \mathrm{E}_{2 \mathrm{D}}^{\varepsilon}[\boldsymbol{z}]
$$

where $a_{S}$ is an adimensional constant. Note that the difference with Koiter's original estimate (1.3) lies in the presence of a boundary term depending on the lateral wave-length $\ell$. We also prove (Theorem A.1) the following bound for the difference between the energies of $z$ and $\mathrm{U} z$ :

$$
\left|\mathrm{E}_{3 \mathrm{D}}^{\varepsilon}[\mathrm{U} \boldsymbol{z}]-\mathrm{E}_{2 \mathrm{D}}^{\varepsilon}[\boldsymbol{z}]\right| \leq c_{S}\left(\frac{\varepsilon}{R}+\frac{\varepsilon^{2}}{L^{2}}\right) \mathrm{E}_{2 \mathrm{D}}^{\varepsilon}[\boldsymbol{z}],
$$

where $c_{S}$ is an adimensional constant depending only on $S$.

Under the same assumption on $L$ as above, we deduce from (1.5) and (1.6) the relative energy estimate for $\varepsilon$ small enough:

$$
\frac{\mathrm{E}_{3 \mathrm{D}}^{\varepsilon}[\boldsymbol{u}-\mathrm{U} \boldsymbol{z}]}{\mathrm{E}_{3 \mathrm{D}}^{\varepsilon}[\mathrm{U} \boldsymbol{z}]} \leq 2 a_{S}\left(\frac{\varepsilon}{\ell}+\frac{\varepsilon^{2}}{\mathrm{r}^{2}}+\frac{\varepsilon^{2}}{L^{2}}\right) .
$$

In the cases of plates and elliptic shells, the behavior of the three characteristic lengths $L, \ell$, and $r$ with respect to the thickness $\varepsilon$ can be made explicit for families of solutions corresponding to a standard load: For plates, $r=+\infty$ and the two wave-lengths $L$ and $\ell$ are $\mathcal{O}(1)$, whereas for elliptic shells, $\ell$ is $\mathcal{O}(1)$, and $L$ is $\mathcal{O}\left(\sqrt{\varepsilon R_{\partial}}\right)$ where $R_{\partial}$ is the curvature radius along the boundary of $S$. In both cases our general estimate (1.5) gives back the optimal estimate (1.4).

Our result can be viewed as an a posteriori estimation of the modeling error $\boldsymbol{u}-\mathrm{U} \boldsymbol{z}$ by means of the 2D solution $z$. It is general with respect to the geometry of the mid-surface $S$ which can be hyperbolic or parabolic as well. However, the question of the behavior of the wave-lengths $L$ and $\ell$ in the latter cases is still open. 
Estimate (1.5) is not only an asymptotic result: It is indeed valid for all $\varepsilon$ for which the domain $\Omega^{\varepsilon}$ is well-defined as a shell. Likewise, it does not specify any special dependency (or independence) of the mid-surface $S$ or the loading $f$ with respect to $\varepsilon$. In particular, the mid-surface $S$ can be embedded in a family depending on $\varepsilon$, as exemplified by the case of shallow shells.

Our result does not require more regularity than $L^{2}$ for the loading $f$. However, for the right-hand side of (1.5) to be finite, we need that the mean value $\boldsymbol{g}$ of $\boldsymbol{f}$ across the shell is more regular. Still, loadings like those of $[22,34]$ can be considered.

\section{1.e Plan of the Paper}

In $\S 2$, we introduce problems $\left(P_{3 \mathrm{D}}\right)$ and $\left(P_{2 \mathrm{D}}\right)$, the different characteristic lengths, the reconstruction operator, and state our results. In $\S 3$, we start the proof of our main estimate: Our strategy is rather classical, see [29, 30], and consists of two main steps:

(i) A variational type estimate of the energy scalar product of the difference $\boldsymbol{u}-U \boldsymbol{z}$ against all displacements $\boldsymbol{v}$ satisfying the clamped boundary conditions,

(ii) An energy estimate of a correcting displacement $\boldsymbol{u}^{\text {cor }}$ constructed so that $\mathrm{U} \boldsymbol{z}+\boldsymbol{u}^{\text {cor }}$ also satisfies the clamped boundary conditions.

For step $(i)$, instead of the reconstructed displacement $\mathrm{U} z$ alone, we consider a more elaborate reconstruction operator. Such a method was initiated in the early works of KOITER, JOHN and NAGHDI see [26, 27, 23, 32]. In order to bound efficiently the remainder by a Korn like estimate, we need a formal expansion of $\boldsymbol{u}$ up to $\varepsilon^{4}$ : For this we take the first five terms of the formal series expansion of $\boldsymbol{u}$ provided in [19], and recalled in $\S 4$.

In $\S 5$, we prove adimensional a priori estimates for norms of $\boldsymbol{z}$ by norms of its membrane and bending strain tensors $\gamma$ and $\rho$. Using the wave-lengths, these latter norms can be compared to the energy of $\boldsymbol{z}$. This formalism allows to obtain intrinsic adimensional estimates. With all these tools at hand, we perform the step $(i)$ in $\S 6$ which is the most delicate part of our work, partly due to the requirement of adimensionality.

Step (ii) is performed in $\S 7$ by an explicit construction. The corrector term only depends on the trace of $\mathrm{U} z$ along the boundary and mimics the three dimensional boundary layer behavior appearing in the situations of plates and in the general formal analysis of [19]. In $\S 8$, we show that our estimate is optimal for plates, for shallow shells in the sense of [13] and for elliptic shells. For this, we rely on the sharp asymptotic expansions provided in [15], [4] and [20]. Finally, we prove in Appendix Appendix A the energy estimate (1.6).

\section{Statement OF RESUltS}

In this section, we now formulate precisely our assumptions, the definitions of problems $\left(P_{3 \mathrm{D}}\right)$ and $\left(P_{2 \mathrm{D}}\right)$ and of the different lengths occurring in estimates (1.5) and (1.6), and we state our main results. We use everywhere the convention of repeated indices for the contraction of tensors. 


\section{A THE THREE-DIMENSIONAL PROBLEM}

In all this work $\left\{\Omega^{\varepsilon}\right\}_{\varepsilon \leq \varepsilon_{0}}$ denotes a family of elastic shells defined for $\varepsilon_{0}$ sufficiently small, made with an isotropic and homogeneous material characterized by its two Lamé coefficients $\lambda$ and $\mu$. The mid-surface of the shell is represented by a smooth 2-manifold $S$ embedded in $\mathbb{R}^{3}$, compact with non-empty boundary $\partial S$. We stress that no other assumption is made on the geometry of the surface $S$. In particular, its main curvatures may have different signs, or even be zero, in which case the shell is a plate. The domain $\Omega^{\varepsilon}$ is then the image of the manifold $\mathcal{Q}^{\varepsilon}:=S \times(-\varepsilon, \varepsilon)$ by the application $\Phi$ :

$$
\mathcal{Q}^{\varepsilon} \ni\left(P, x_{3}\right) \mapsto \Phi\left(P, x_{3}\right)=P+x_{3} \boldsymbol{n}(P) \in \Omega^{\varepsilon} \subset \mathbb{R}^{3}
$$

where $\boldsymbol{n}$ is a continuous unit normal field on $S$. For a function $f$ defined on $\Omega^{\varepsilon}$, we have

$$
\int_{\Omega^{\varepsilon}} f\left(t^{1}, t^{2}, t^{3}\right) \mathrm{d} t^{1} \mathrm{~d} t^{2} \mathrm{~d} t^{3}=\int_{\mathcal{Q}^{\varepsilon}} f\left(\Phi\left(P, x_{3}\right)\right)\left(1+h\left(x_{3}\right)\right) \mathrm{d} S \mathrm{~d} x_{3},
$$

where $\left\{t^{i}\right\}$ denote a Cartesian coordinate system in $\mathbb{R}^{3}$, and where $\left|h\left(x_{3}\right)\right|<1 / 2$ (provided $\varepsilon_{0}$ is small enough).

The shell has two faces $\Gamma_{ \pm}^{\varepsilon}$ corresponding to $S \times\{ \pm \varepsilon\}$ and a lateral boundary $\Gamma_{0}^{\varepsilon}$ corresponding to $\partial S \times(-\varepsilon, \varepsilon)$. The boundary conditions applied to the shell are the free traction conditions on the two faces $\Gamma_{ \pm}^{\varepsilon}$ and the clamped conditions on $\Gamma_{0}^{\varepsilon}$. The space of admissible displacements is then

$$
V\left(\Omega^{\varepsilon}\right)=\left\{\boldsymbol{u} \in \mathrm{H}^{1}\left(\Omega^{\varepsilon}\right)^{3} \quad \mid \quad \boldsymbol{u}=0 \quad \text { on } \quad \Gamma_{0}^{\varepsilon}\right\} .
$$

If $\boldsymbol{u}$ and $\boldsymbol{v}$ are two displacements on $\Omega^{\varepsilon}$, we define the energy scalar product

$$
a_{3 \mathrm{D}}^{\varepsilon}(\boldsymbol{u}, \boldsymbol{v})=\int_{\Omega^{\varepsilon}} A^{i j k \ell} e_{i j}(\boldsymbol{u}) e_{k \ell}(\boldsymbol{v}) \mathrm{d} t^{1} \mathrm{~d} t^{2} \mathrm{~d} t^{3},
$$

where

$$
A^{i j k \ell}=\lambda \delta^{i j} \delta^{k \ell}+\mu\left(\delta^{i k} \delta^{j \ell}+\delta^{i \ell} \delta^{j k}\right)
$$

is the rigidity tensor of the material, with the Kronecker tensor $\delta^{i j}$. The tensor $e_{i j}(\boldsymbol{v})=$ $\frac{1}{2}\left(\partial_{i} v_{j}+\partial_{j} v_{i}\right)$ is the strain tensor in Cartesian coordinates, where $\partial_{i}$ denotes the derivative with respect to $t^{i}$. The associated quadratic three-dimensional energy of a displacement $\boldsymbol{v}$ is then:

$$
\mathrm{E}_{3 \mathrm{D}}^{\varepsilon}[\boldsymbol{v}]:=a_{3 \mathrm{D}}^{\varepsilon}(\boldsymbol{v}, \boldsymbol{v})
$$

For a load $\boldsymbol{f} \in \mathrm{L}^{2}\left(\Omega^{\varepsilon}\right)^{3}$, the "exact solution" $\boldsymbol{u}$ is the displacement solution of the variational problem :

$\left(P_{3 \mathrm{D}}\right) \quad$ Find $\boldsymbol{u} \in V\left(\Omega^{\varepsilon}\right)$ such that $\forall \boldsymbol{v} \in V\left(\Omega^{\varepsilon}\right), \quad a_{3 \mathrm{D}}^{\varepsilon}(\boldsymbol{u}, \boldsymbol{v})=\int_{\Omega^{\varepsilon}} \boldsymbol{f} \cdot \boldsymbol{v} \mathrm{d} t^{1} \mathrm{~d} t^{2} \mathrm{~d} t^{3}$. 


\section{B NORMAL COORDINATES AND TENSORS}

The shell $\Omega^{\varepsilon}$ is diffeomorphic to the manifold $\mathcal{Q}^{\varepsilon}$ via the application (2.1). Any local coordinate system $\left(x_{\sigma}\right)$ on $S$ yields a coordinate system $\left(x_{\sigma}, x_{3}\right)$ on $\mathcal{Q}^{\varepsilon}$ and thus an atlas on $S$ provides an atlas on $\Omega^{\varepsilon}$ whose local maps are $U \times(-\varepsilon, \varepsilon)$ where $U$ are the maps of the atlas on $S$. Such a coordinate system is called normal coordinate system, and induces a basis for tensor fields on $\Omega^{\varepsilon}$.

This implies that every tensor on $\Omega^{\varepsilon}$ can be decomposed into several two-dimensional tensors depending smoothly on $x_{3}$ and living on $S$. Typically, any displacement (i.e. a 1 -form on $\Omega^{\varepsilon}$ ) $\boldsymbol{v}$ splits into

(i) a surface displacement $\left(v_{\sigma}\right)$, which means that $x_{3} \mapsto\left(v_{\sigma}\left(x_{3}\right)\right)$ takes its values in 1 -forms on $S$.

(ii) a function $v_{3}$, in other words $x_{3} \mapsto v_{3}\left(x_{3}\right)$ takes its values in functions on $S$.

On the same way, for each fixed $x_{3}$, the strain tensor $e_{i j}$ splits into: $e_{33}$, which is a function on $S,\left(e_{\sigma 3}\right)$ which is a covariant tensor of order 1 on $S$, and $\left(e_{\alpha \beta}\right)$ which is a covariant tensor of order 2 on $S$. These three surfacic tensors depend smoothly on $x_{3}$.

We denote by $\boldsymbol{a}=\left(a_{\alpha \beta}\right)$ the metric tensor on $S$ induced by the ambient metric in $\mathbb{R}^{3}$, and by $\boldsymbol{b}=\left(b_{\alpha \beta}\right)$ the curvature tensor on $S$ (see e.g. [17, 38]). These tensors are symmetric covariant tensors of order 2 . Moreover, the metric tensor induces an isomorphism between covariant and contravariant tensors. For instance, the tensor $b_{\sigma}^{\alpha}$ is defined by $b_{\sigma}^{\alpha}=a^{\alpha \beta} b_{\beta \sigma}$, with the inverse $a^{\alpha \beta}$ of the metric tensor. We also denote by $\mathrm{D}_{\alpha}$ the covariant derivative induced by the Riemannian metric $a_{\alpha \beta}$ on $S$.

Let us recall the definition of the Sobolev norm of a tensor on a manifold. Consider a covariant tensor field $\tau$ of order $k$ on $S$. In a local coordinate system, we denote by $\tau_{\alpha_{1} \alpha_{2} \cdots \alpha_{k}}$ its components. The norm $|\boldsymbol{\tau}|$ of $\boldsymbol{\tau}$ at a fixed point $P \in S$ is defined as $|\boldsymbol{\tau}|=$ $\left(\tau^{\alpha_{1} \alpha_{2} \cdots \alpha_{k}} \tau_{\alpha_{1} \alpha_{2} \cdots \alpha_{k}}\right)^{1 / 2}$ where $\tau^{\alpha_{1} \alpha_{2} \cdots \alpha_{k}}$ is the contravariant tensor associated with $\boldsymbol{\tau}$ using the metric tensor, as explained above. The expression of the norm $|\boldsymbol{\tau}|$ is independent of local coordinate systems and makes sense for tensors of any type, since it depends only on the order of the tensor and not on its representation as covariant or contravariant tensor. We have for example $|\boldsymbol{b}|^{2}=b^{\alpha \beta} b_{\alpha \beta}=b_{\beta}^{\alpha} b_{\alpha}^{\beta}$ so that can write $|\boldsymbol{b}|=\left|b_{\alpha \beta}\right|=\left|b_{\alpha}^{\beta}\right|$.

The $\mathrm{L}^{2}$ norm $|\boldsymbol{\tau}|_{0 ; S}$ of $\boldsymbol{\tau}$ is defined as $\left(\int_{S}|\boldsymbol{\tau}|^{2} \mathrm{~d} S\right)^{1 / 2}$. For $n \in \mathbb{N}$, we denote by $\mathrm{D}_{[n]} \boldsymbol{\tau}$ the tensor of order $k+n$ with components $\mathrm{D}_{\beta_{1}} \cdots \mathrm{D}_{\beta_{n}} \tau_{\alpha_{1} \alpha_{2} \cdots \alpha_{k}}$ in a local coordinate system. The semi norm of order $n$ of $\tau$ is thus

$$
|\boldsymbol{\tau}|_{n ; S}=\left|\mathrm{D}_{[n]} \boldsymbol{\tau}\right|_{0 ; S}
$$

As the surface $S$ is smooth, this expression makes sense on $S$ for all $n$, and does not depend on a choice of a local coordinate system. We define similarly the semi-norms $|\boldsymbol{\tau}|_{n ; \partial S}$ on the lateral boundary $\partial S$.

In the following, we denote by $\mathbf{H}^{k}(S)$ the space of 1 -form fields $\left(z_{\sigma}\right)$ such that $\left|z_{\sigma}\right|_{n ; S}<\infty$ for $n=0, \ldots, k$, and by $\mathrm{H}^{k}(S)$ the corresponding space for functions. We also use the notation $\Sigma(S):=\Gamma\left(T_{1} \bar{S}\right) \times \mathscr{C}^{\infty}(\bar{S})$ where $\Gamma\left(T_{1} \bar{S}\right)$ denotes the space of smooth 1-form fields on $\bar{S}$ (see e.g. [19] for details). 


\section{C THE TWO-DIMENSIONAL PROBLEM}

The Koiter operator on $S$ is defined as $\mathrm{K}(\varepsilon)=\mathrm{M}+\varepsilon^{2} \mathrm{~B}$ where $\mathrm{M}$ is the membrane operator and $\mathrm{B}$ the bending operator. Both of them involve the rigidity tensor $M^{\alpha \beta \sigma \delta}$ corresponding to the modified Lamé constants $\tilde{\lambda}=2 \lambda \mu /(\lambda+2 \mu)$ and $\mu$ :

$$
M^{\alpha \beta \sigma \delta}=\tilde{\lambda} a^{\alpha \beta} a^{\sigma \delta}+\mu\left(a^{\alpha \sigma} a^{\delta \beta}+a^{\alpha \delta} a^{\beta \sigma}\right) .
$$

Both operators $\mathrm{M}$ and $\mathrm{B}$ act on spaces of $\boldsymbol{z}=\left(z_{\sigma}, z_{3}\right)$ where $\left(z_{\sigma}\right)$ is a 1 -form on $S$ and $z_{3}$ a function on $S$. The target space contains elements of the form $\boldsymbol{g}=\left(g_{\sigma}, g_{3}\right)$ where $\left(g_{\sigma}\right)$ is a 1 -form on $S$ and $g_{3}$ a function on $S$. Typical spaces for $\boldsymbol{z}$ are $\mathbf{H}^{1} \times \mathrm{L}^{2}(S)$ and $\mathbf{H}^{1} \times \mathrm{H}^{2}(S)$.

The operator $\mathrm{M}$ is the operator associated with the bilinear form $a_{\mathrm{M}}$ defined for any $\boldsymbol{z}=\left(z_{\sigma}, z_{3}\right)$ and $\boldsymbol{\eta}=\left(\eta_{\sigma}, \eta_{3}\right)$ in $\mathbf{H}^{1} \times \mathrm{L}^{2}(S)$ by

$$
(\boldsymbol{z}, \boldsymbol{\eta}) \mapsto a_{\mathrm{M}}(\boldsymbol{z}, \boldsymbol{\eta})=\int_{S} M^{\alpha \beta \sigma \delta} \gamma_{\alpha \beta}(\boldsymbol{z}) \gamma_{\sigma \delta}(\boldsymbol{\eta}) \mathrm{d} S
$$

where the membrane strain tensor field

$$
\gamma_{\alpha \beta}(\boldsymbol{z})=\frac{1}{2}\left(\mathrm{D}_{\alpha} z_{\beta}+\mathrm{D}_{\beta} z_{\alpha}\right)-b_{\alpha \beta} z_{3}
$$

is the change of metric tensor.

The operator $\mathrm{B}$ is associated with the bilinear form $a_{\mathrm{B}}$ defined for any $\boldsymbol{z}$ and $\boldsymbol{\eta}$ in $\mathbf{H}^{1} \times \mathrm{H}^{2}(S)$ by

$$
(\boldsymbol{z}, \boldsymbol{\eta}) \mapsto a_{\mathrm{B}}(\boldsymbol{z}, \boldsymbol{\eta})=\frac{1}{3} \int_{S} M^{\alpha \beta \sigma \delta} \rho_{\alpha \beta}(\boldsymbol{z}) \rho_{\sigma \delta}(\boldsymbol{\eta}) \mathrm{d} S
$$

where

$$
\rho_{\alpha \beta}(\boldsymbol{z})=\mathrm{D}_{\alpha} \mathrm{D}_{\beta} z_{3}-b_{\alpha}^{\sigma} b_{\sigma \beta} z_{3}+b_{\alpha}^{\sigma} \mathrm{D}_{\beta} z_{\sigma}+\mathrm{D}_{\alpha} b_{\beta}^{\sigma} z_{\sigma}
$$

is the change of curvature tensor.

The two-dimensional energy scalar product is defined for $\boldsymbol{z}, \boldsymbol{\eta} \in \mathbf{H}^{1} \times \mathrm{H}^{2}(S)$ by

$$
a_{2 \mathrm{D}}^{\varepsilon}(\boldsymbol{z}, \boldsymbol{\eta})=a_{\mathrm{M}}(\boldsymbol{z}, \boldsymbol{\eta})+\varepsilon^{2} a_{\mathrm{B}}(\boldsymbol{z}, \boldsymbol{\eta}) .
$$

This bilinear form is associated with the Koiter operator $K(\varepsilon)=M+\varepsilon^{2} B$. The physical quadratic energy associated with a displacement $\boldsymbol{z}$ is defined as:

$$
\mathrm{E}_{2 \mathrm{D}}^{\varepsilon}[\boldsymbol{z}]:=2 \varepsilon a_{2 \mathrm{D}}^{\varepsilon}(\boldsymbol{z}, \boldsymbol{z}) .
$$

The right-hand side $\boldsymbol{g}=\left(g_{\sigma}, g_{3}\right)$ of the two-dimensional problem $\left(P_{2 \mathrm{D}}\right)$ is defined as

$$
\boldsymbol{g}(P)=\frac{1}{2 \varepsilon} \int_{-\varepsilon}^{\varepsilon} \boldsymbol{f}\left(P, x_{3}\right) \mathrm{d} x_{3}, \quad P \in S
$$

The admissible two-dimensional displacement space is $\mathbf{H}_{0}^{1} \times \mathrm{H}_{0}^{2}(S)$. The two-dimensional problem then writes:

$$
\text { Find } \boldsymbol{z} \in \mathbf{H}_{0}^{1} \times \mathrm{H}_{0}^{2}(S) \text { such that }
$$

$$
\forall \boldsymbol{\eta} \in \mathbf{H}_{0}^{1} \times \mathrm{H}_{0}^{2}(S), \quad a_{2 \mathrm{D}}^{\varepsilon}(\boldsymbol{z}, \boldsymbol{\eta})=\int_{S}\left(a^{\alpha \beta} g_{\alpha} \eta_{\beta}+g_{3} \eta_{3}\right) \mathrm{d} S .
$$

We define the residual load as

$$
\boldsymbol{f}^{\mathrm{rem}}\left(P, x_{3}\right):=\boldsymbol{f}\left(P, x_{3}\right)-\boldsymbol{g}(P) .
$$




\section{D Physical Dimensions}

In Table 1 we collect the physical dimensions of the objects present in problems $\left(P_{3 \mathrm{D}}\right)$ and $\left(P_{2 \mathrm{D}}\right)$.

\begin{tabular}{|c|c|c|c|c|}
\hline Physical object & $\begin{array}{c}\text { Notation } \\
\text { 3D }\end{array}$ & $\begin{array}{c}\text { Dimension } \\
\text { 3D }\end{array}$ & $\begin{array}{c}\text { Notation } \\
\text { 2D }\end{array}$ & $\begin{array}{c}\text { Dimension } \\
\text { 2D }\end{array}$ \\
\hline Displacement & $\boldsymbol{u}$ & $m$ & $\boldsymbol{z}$ & $m$ \\
\hline Volume force & $\boldsymbol{f}$ & $N . m^{-3}$ & $\boldsymbol{g}$ & $N . m^{-3}$ \\
\hline Energy & $\mathrm{E}_{3 \mathrm{D}}[\boldsymbol{u}]$ & $N . m$ (Joule) & $\mathrm{E}_{2 \mathrm{D}}[\boldsymbol{z}]$ & $N . m$ (Joule) \\
\hline Curvature & & & $b_{\alpha}^{\beta}$ & $m^{-1}$ \\
\hline Covariant derivative & & & $\mathrm{D}_{\alpha}$ & $m^{-1}$ \\
\hline $\begin{array}{c}\text { Deformation rate } \\
\text { Change of metric }\end{array}$ & $e_{i j}(\boldsymbol{u})$ & Adimensional & $\gamma_{\alpha \beta}(\boldsymbol{z})$ & Adimensional \\
\hline Change of curvature & & & $\rho_{\alpha \beta}(\boldsymbol{z})$ & $m^{-1}$ \\
\hline Material coefficients & $E, \lambda, \mu$ & $N . m^{-2}$ (Pascal) & $E, \tilde{\lambda}, \mu$ & $N . m^{-2}$ (Pascal) \\
\hline
\end{tabular}

Table 1. Physical dimensions

Here, $E$ denotes the Young modulus of the material. We recall the formulas

$$
E=\frac{\mu(3 \lambda+2 \mu)}{\lambda+\mu} \quad \text { and } \quad \nu=\frac{\lambda}{2(\lambda+\mu)},
$$

where $\nu$ is the adimensional Poisson coefficient. Conversely

$$
\lambda=\frac{E \nu}{(1+\nu)(1-2 \nu)} \quad \text { and } \quad \mu=\frac{E}{2(1+\nu)} .
$$

\section{E WAVE LENGTHS}

Before defining wave lengths attached to the solution $z$ of $\left(P_{2 \mathrm{D}}\right)$, we introduce a sequence of characteristic quantities depending on the curvature tensor of $S$.

Definition 2.1 (i) We set $\kappa_{0}=1$ and define recursively for $j \geq 1$ the numbers $\kappa_{j}$ by:

$$
\kappa_{1}=\max _{P \in \bar{S}}|\boldsymbol{b}| \quad \text { and } \quad \kappa_{j}=\max \left\{\kappa_{j-1}, \max _{P \in \bar{S}}\left|\mathrm{D}_{[j-1]} \boldsymbol{b}\right|^{1 / j}\right\} \quad \text { for } \quad j \geq 2 \text {, }
$$

where $\boldsymbol{b}$ is the curvature tensor. For any $j \geq 1$, the constants $\kappa_{j}$ have the dimension of the inverse of a length. 
(ii) For any tensor field on $S$ let for all $n \in \mathbb{N}$ the semi-norm $|\boldsymbol{\tau}|_{n ; S}^{(\boldsymbol{b})}$ be defined by*

$$
|\boldsymbol{\tau}|_{n ; S}^{(\boldsymbol{b})}=\left(\sum_{j=0}^{n} \kappa_{j}^{2 j}|\boldsymbol{\tau}|_{n-j ; S}^{2}\right)^{1 / 2}
$$

With the definition (2.15) we have $\kappa_{1}=1 / R$ where $1 / R$ is the maximum principal curvature of $S$. Since the covariant derivative has the dimension of the inverse of a length, we see that all the terms in the sum of the right-hand side of (2.16) have the same dimension. In the case of plates, we have $\kappa_{j}=0$ for $j \geq 1$, and hence $|\boldsymbol{\tau}|_{n ; S}^{(\boldsymbol{b})}=|\boldsymbol{\tau}|_{n ; S}$.

Definition 2.2 An operator $\mathrm{L}$ acting on tensor spaces on $S$ is said to be $\boldsymbol{b}$-homogeneous of degree $n$ if it is a linear combination with adimensional coefficients of contractions of tensors of the form $B_{1} \cdots B_{n}$ where each $B_{j}$ is either the covariant derivative $\mathrm{D}_{\sigma}$ or the curvature tensor $b_{\alpha \beta}$.

Note that the operators $\boldsymbol{z} \mapsto \gamma_{\alpha \beta}(\boldsymbol{z})$ and $\boldsymbol{z} \mapsto \rho_{\alpha \beta}(\boldsymbol{z})$ are $\boldsymbol{b}$-homogeneous of degree 1 and 2 respectively. Similarly the membrane is $\boldsymbol{b}$-homogeneous of degree 2 , which means that both surfacic and transverse components are $\boldsymbol{b}$-homogeneous of degree 2 , and the bending operator is $\boldsymbol{b}$-homogeneous of degree 4 .

This definition is motivated by the following lemma:

Lemma 2.3 Let $\mathrm{L}$ be a $\boldsymbol{b}$-homogeneous operator of degree $n$ acting on tensors $\boldsymbol{\tau}$ of order $k$, ans let $s \in \mathbb{N}$. Then there exists an adimensional constant $A$ such that,

$$
\forall \boldsymbol{\tau}, \quad|\mathrm{L} \boldsymbol{\tau}|_{s ; S}^{(\boldsymbol{b})} \leq A|\boldsymbol{\tau}|_{s+n ; S}^{(\boldsymbol{b})}
$$

Let $\gamma$ and $\rho$ denote the membrane and bending strain tensors of the solution $z$ of problem $\left(P_{2 \mathrm{D}}\right)$. With our notations, we can reformulate Koiter's definition of the quantity $L$ in $[25,26]$ as "the wave length of the deformation pattern of shell theory, defined by the order of magnitude relations $\mathrm{D}_{[1]} \gamma=\mathcal{O}(\gamma / L)$ and $\mathrm{D}_{[1]} \boldsymbol{\rho}=\mathcal{O}(\boldsymbol{\rho} / L)$."

Without being exactly the same, our definitions retain the idea of inverse inequalities for the membrane and bending strain tensors $\gamma$ and $\rho$.

Definition 2.4 For $\boldsymbol{z} \in \Sigma(S)$ we denote by $\gamma=\gamma_{\alpha \beta}(\boldsymbol{z})$ and $\boldsymbol{\rho}=\rho_{\alpha \beta}(\boldsymbol{z})$ the membrane and bending strain tensors associated with $z$. We set $L_{0}=1$ and for all $k \geq 1$, we define the global wave length $L_{k}$ of $z$ as the largest constant such that there holds, for $j=1, \ldots, k$

$$
|\gamma|_{j ; S} \leq L_{k}^{-j}|\gamma|_{0 ; S} \quad \text { and } \quad|\boldsymbol{\rho}|_{j ; S} \leq L_{k}^{-j}|\boldsymbol{\rho}|_{0 ; S}
$$

${ }^{*}$ We could have introduce factorial normalization terms in the definitions (2.15) and (2.6). This could in principle lead to analytic estimates in the case where $S$ is analytic. In this situation, $\kappa_{j}$ would tend to the analytic radius of convergence of $\boldsymbol{b}$ when $j \rightarrow \infty$. 
Note that $L_{1} \geq L_{2} \geq \cdots$, and that $L_{k}$ can be equivalently defined by requiring (2.17) for $j=k$ only. We have, by definition:

$$
\left(|\boldsymbol{\gamma}|_{n ; S}^{(\boldsymbol{b})}\right)^{2} \leq|\gamma|_{0 ; S}^{2} \sum_{j+k=n} L_{k}^{-2 k} \kappa_{j}^{2 j} \quad \text { and } \quad\left(|\boldsymbol{\rho}|_{n ; S}^{(\boldsymbol{b})}\right)^{2} \leq|\boldsymbol{\rho}|_{0 ; S}^{2} \sum_{j+k=n} L_{k}^{-2 k} \kappa_{j}^{2 j} .
$$

We now define a similar wave length, now for the norms on the boundary $\partial S$.

Definition 2.5 With $\gamma$ and $\rho$ as in Definition 2.4, we define $\ell$ as the largest constant such that there holds

$$
\begin{array}{lll}
|\gamma|_{0 ; \partial S} \leq \ell^{-1 / 2}|\gamma|_{0 ; S} & \text { and } & |\boldsymbol{\rho}|_{0 ; \partial S} \leq \ell^{-1 / 2}|\boldsymbol{\rho}|_{0 ; S} \\
|\gamma|_{1 ; \partial S} \leq \ell^{-3 / 2}|\gamma|_{0 ; S} & \text { and } & |\boldsymbol{\rho}|_{1 ; \partial S} \leq \ell^{-3 / 2}|\boldsymbol{\rho}|_{0 ; S}
\end{array}
$$

Note that the quantity $\ell$ has also the dimension of a length.

\section{F KORN INEQUALITIES}

We now define a length $D$ through Korn inequalities $\Omega^{\varepsilon}$ involving the Young modulus $E$ and the thickness $\varepsilon$ of the shell.

Proposition 2.6 There exists a constant D independent on $\varepsilon$, having the dimension of a length, such that for all $\boldsymbol{v} \in V\left(\Omega^{\varepsilon}\right)$ defined in (2.3), we have

$$
\begin{aligned}
\|\boldsymbol{v}\|_{\mathrm{L}^{2}\left(\Omega^{\varepsilon}\right)}^{2} & \leq \mathrm{D}^{4} E^{-1} \varepsilon^{-2} \mathrm{E}_{3 \mathrm{D}}^{\varepsilon}[\boldsymbol{v}] \\
\left\|\mathrm{D}_{\alpha} \boldsymbol{v}\right\|_{\mathrm{L}^{2}\left(\Omega^{\varepsilon}\right)}^{2}+\left\|\partial_{3} \boldsymbol{v}\right\|_{\mathrm{L}^{2}\left(\Omega^{\varepsilon}\right)}^{2} & \leq \mathrm{D}^{2} E^{-1} \varepsilon^{-2} \mathrm{E}_{3 \mathrm{D}}^{\varepsilon}[\boldsymbol{v}] .
\end{aligned}
$$

Proof. We perform the scaling $X_{3}=\varepsilon^{-1} x_{3}$ mapping the manifold $\mathcal{Q}^{\varepsilon}$ to the manifold $\mathcal{Q}:=S \times(-1,1)$. In $\mathcal{Q}$, the expression of the deformation tensor $e_{i j}(\varepsilon)(\boldsymbol{v})$ is obtained like in [12] by changing $\partial_{x_{3}}$ to $\varepsilon^{-1} \partial_{X_{3}}$. The variable $X_{3}$ and the derivative $\partial_{X_{3}}$ are adimensional, as well as the tensor $e_{i j}(\varepsilon)(\boldsymbol{v})$.

The lateral boundary $\Gamma_{0}=\partial S \times(-1,1)$ is the image of $\Gamma_{0}^{\varepsilon}$ by the scaling. We define the space $V(\mathcal{Q})$ as the set of $\boldsymbol{v} \in \mathrm{H}(\mathcal{Q})^{3}$ such that $\left.\boldsymbol{v}\right|_{\Gamma_{0}}=0$. On the manifold $\mathcal{Q}$, the following inequalities hold (see [12]): For all $v \in V(\mathcal{Q})$,

$$
\|\boldsymbol{v}\|_{\mathrm{L}^{2}(\mathcal{Q})}+\left\|\mathrm{D}_{\alpha} \boldsymbol{v}\right\|_{\mathrm{L}^{2}(\mathcal{Q})} \leq C_{1} \varepsilon^{-1}\left\|e_{i j}(\varepsilon)(\boldsymbol{v})\right\|_{\mathrm{L}^{2}(\mathcal{Q})},
$$

where

$$
\left\|e_{i j}(\varepsilon)(\boldsymbol{v})\right\|_{\mathrm{L}^{2}(\mathcal{Q})}=\left(\sum_{i, j=1,2,3} \int_{\mathcal{Q}} e_{i j}(\varepsilon)(\boldsymbol{v})^{2} \mathrm{~d} S \mathrm{~d} X_{3}\right)^{1 / 2}
$$

where $\mathrm{d} S$ is the volume form on $S$.

The shear deformation tensor is written as the convergent series (see [32, 19]):

$$
e_{\alpha 3}(\varepsilon)(\boldsymbol{v})=\frac{1}{2}\left(\mathrm{D}_{\alpha} v_{3}+\varepsilon^{-1} \partial_{X_{3}} v_{\alpha}\right)+\sum_{k=0}^{\infty} \varepsilon^{j} X_{3}^{j}\left(b^{j+1}\right)_{\alpha}^{\beta} v_{\beta}
$$


where $\left(b^{j}\right)_{\alpha}^{\beta}$ denotes the product of $j$-times the curvature tensor $\boldsymbol{b}$. We thus have

$$
\left\|\partial_{X_{3}} v_{\alpha}\right\|_{L^{2}(\mathcal{Q})} \leq 2 \varepsilon\left\|e_{\alpha 3}(\varepsilon)(\boldsymbol{v})\right\|_{L^{2}(\mathcal{Q})}+\varepsilon\left(\sum_{j=0}^{\infty} \varepsilon^{j} \kappa_{1}^{j+1}\right)\|\boldsymbol{v}\|_{L^{2}(\mathcal{Q})}+\varepsilon\left\|\mathrm{D}_{\alpha} \boldsymbol{v}\right\|_{L^{2}(\mathcal{Q})} .
$$

Combining this estimate with (2.21), we obtain the existence of $C_{2}$, having the dimension of a length, such that

$$
\left\|\partial_{X_{3}} \boldsymbol{v}\right\|_{\mathrm{L}^{2}(\mathcal{Q})} \leq C_{2}\left\|e_{i j}(\varepsilon)(\boldsymbol{v})\right\|_{\mathrm{L}^{2}(\mathcal{Q})},
$$

which improves the corresponding estimate in [12]. Scaling back to $\mathcal{Q}^{\varepsilon}$ and using (2.2), we get the estimates for the squared norms:

$$
\begin{aligned}
\|\boldsymbol{v}\|_{\mathrm{L}^{2}\left(\Omega^{\varepsilon}\right)}^{2} & \leq C_{3} \varepsilon^{-2}\left\|e_{i j}(\boldsymbol{v})\right\|_{\mathrm{L}^{2}\left(\Omega^{\varepsilon}\right)}^{2}, \\
\left\|\mathrm{D}_{\alpha} \boldsymbol{v}\right\|_{\mathrm{L}^{2}\left(\Omega^{\varepsilon}\right)}^{2}+\left\|\partial_{3} \boldsymbol{v}\right\|_{\mathrm{L}^{2}\left(\Omega^{\varepsilon}\right)}^{2} & \leq C_{4} \varepsilon^{-2}\left\|e_{i j}(\boldsymbol{v})\right\|_{\mathrm{L}^{2}\left(\Omega^{\varepsilon}\right)}^{2} .
\end{aligned}
$$

The constants $C_{3}$ and $C_{4}$ have the dimensions $m^{4}$ and $m^{2}$ respectively.

Using relations (2.14) we obtain adimensional constants $a$ and $A$ such that

$$
a E \leq \lambda \leq A E \quad \text { and } \quad a E \leq \mu \leq A E
$$

Since $E>0$, there exists an adimensional constant $A_{1}$ such that

$$
\left\|e_{i j}(\boldsymbol{v})\right\|_{\mathrm{L}^{2}\left(\Omega^{\varepsilon}\right)}^{2} \leq A_{1} E^{-1} \mathrm{E}_{3 \mathrm{D}}^{\varepsilon}[\boldsymbol{v}]
$$

Denoting by $\mathrm{D}$ the length $\max \left\{\left(A_{1} C_{3}\right)^{1 / 4},\left(A_{1} C_{4}\right)^{1 / 2}\right\}$, we obtain the result.

\section{G MAIN ENERGY ESTIMATE}

Our main result gives an estimate between the three-dimensional displacement field and the reconstructed displacement. Besides the notations defined in the previous sections, we need one more characteristic length $\mathrm{d}$ of the shell $S$.

Definition 2.7 Let $r$ denote the geodesic distance in $S$ to the boundary $\partial S$, and let $s$ be the arc-length along $\partial S$. We denote by $\mathbf{d}$ the maximal width of the tubular neighborhood in which $(r, s) \in[0, \mathrm{~d}] \times \partial S$ defines a smooth coordinate system.

It is clear that $d$ has the dimension of a length and that $d$ is proportional to the minimum radius of curvature of the boundary $\partial S$ viewed as a sub-manifold of $S$.

Let $\boldsymbol{z}=\left(z_{\sigma}, z_{3}\right)$ be solution of $\left(P_{2 \mathrm{D}}\right)$. With $\boldsymbol{z}$, we associate the three-dimensional displacement $\mathrm{U} z$ defined by the formula (cf. [26])

$$
\mathrm{U} \boldsymbol{z}=\left\{\begin{array}{l}
z_{\sigma}-x_{3}\left(\mathrm{D}_{\sigma} z_{3}+2 b_{\sigma}^{\alpha} z_{\alpha}\right)+x_{3}^{2} b_{\sigma}^{\alpha} \theta_{\alpha}(\boldsymbol{z}) \\
z_{3}-p x_{3} \gamma_{\alpha}^{\alpha}(\boldsymbol{z})+p \frac{x_{3}^{2}}{2} \rho_{\alpha}^{\alpha}(\boldsymbol{z})
\end{array}\right.
$$

where $\theta_{\sigma}(\boldsymbol{z})=\mathrm{D}_{\sigma} z_{3}+b_{\sigma}^{\alpha} z_{\alpha}$ and $p=\lambda(\lambda+2 \mu)^{-1}$. 
Theorem 2.8 Let $\boldsymbol{u}$ and $\boldsymbol{z}$ be the solutions of $\left(P_{3 \mathrm{D}}\right)$ and $\left(P_{2 \mathrm{D}}\right)$ respectively, and let $\mathrm{U} \boldsymbol{z}$ be the displacement (2.23). Let $L=L_{4}$ and $\ell$ be defined in Definitions 2.4-2.5, $r=1 / \kappa_{5}$ given by Definition 2.1. Assume that $\varepsilon \leq \varepsilon_{0}<\min \{\mathrm{r}, \mathrm{d}\}$ and that

$$
\sup _{\varepsilon \leq \varepsilon_{0}}\left|\frac{\varepsilon}{\ell}+\frac{\varepsilon^{2}}{L^{2}}\right| \leq M<\infty .
$$

Then the following estimate holds:

$$
\begin{aligned}
\mathrm{E}_{3 \mathrm{D}}^{\varepsilon}[\boldsymbol{u}-\mathrm{U} \boldsymbol{z}] \leq a_{S}\left(B_{S}(\varepsilon ; \boldsymbol{z}) \mathrm{E}_{2 \mathrm{D}}^{\varepsilon}[\boldsymbol{z}]+\mathrm{D}^{2} E^{-1}\left\|\boldsymbol{f}^{\mathrm{rem}}\right\|_{\mathrm{L}^{2}\left(\Omega^{\varepsilon}\right)}^{2}\right) \\
\text { with } B_{S}(\varepsilon ; \boldsymbol{z})=\frac{\varepsilon}{\ell}+\frac{\varepsilon^{2}}{\mathrm{r}^{2}}+\frac{\varepsilon^{2}}{L^{2}}+\frac{\varepsilon^{4} \mathrm{D}^{2}}{L^{6}}
\end{aligned}
$$

where $E$ is the Young modulus, $f^{\text {rem }}$ is the residual load (2.12) and D the constant appearing in Proposition 2.6. The constant $a_{S}$ is an adimensional constant such that

$$
a_{S} \leq b_{S}\left(1+M^{3}\right)
$$

where $b_{S}$ is an adimensional constant depending only on $S$.

Remark 2.9 For the quantity $L=L_{4}$ to be finite, we need that the mean value $\boldsymbol{g}$ of $\boldsymbol{f}$ across the shell is more regular. The ellipticity of the Koiter operator in the sense of AGMON, Douglis, Nirenbreg [1] clearly implies that $L_{4}$ is finite if $\boldsymbol{g}$ belongs to $\mathrm{H}^{3}(S)^{3}$ but the control of $L_{4}$ with respect to the small parameter $\varepsilon$ is in general very difficult (see [2, 29]) and depends on the geometry of the mid-surface $S$ and its regularity (see in particular [7] for non smooth mid-surfaces).

Remark 2.10 If the loading belongs to $\mathrm{H}^{1}\left(\Omega^{\varepsilon}\right)$, owing to the estimate

$$
\left\|\boldsymbol{f}^{\mathrm{rem}}\right\|_{\mathrm{L}^{2}\left(\Omega^{\varepsilon}\right)}^{2} \leq A_{S} \varepsilon^{2}\|\nabla \boldsymbol{f}\|_{\mathrm{L}^{2}\left(\Omega^{\varepsilon}\right)}^{2}
$$

with an adimensional constant $A_{S}$, the contribution of $\boldsymbol{f}^{\text {rem }}$ to the bound of $\mathrm{E}_{3 \mathrm{D}}^{\varepsilon}[\boldsymbol{u}-\mathrm{U} \boldsymbol{z}]$ is of higher order. If $\boldsymbol{g}=0$, the 2D displacement $\boldsymbol{z}$ is also 0 , and we are in a regime of higher order answers (see [5, 14] for the case of plates).

\section{METHOD OF PROOF FOR THE MAIN ESTIMATE}

To prove (2.24), we have to take lateral Dirichlet boundary conditions on $\Gamma_{0}^{\varepsilon}$ into account. As $\mathrm{U} z$ does not satisfy these boundary conditions in general, we will add a correction term $\boldsymbol{u}^{\text {cor }}$ to it so that $\mathrm{U} \boldsymbol{z}+\boldsymbol{u}^{\text {cor }}$ is zero on $\Gamma_{0}^{\varepsilon}$.

The plan of the proof of (2.24) originates from the following

Theorem 3.1 Let $\boldsymbol{u}$ be solution of problem $\left(P_{3 \mathrm{D}}\right), \boldsymbol{z}$ the solution of problem $\left(P_{2 \mathrm{D}}\right)$ and $\boldsymbol{u}^{\text {cor }}$ constructed so that $\mathrm{U} \boldsymbol{z}+\boldsymbol{u}^{\text {cor }} \in V\left(\Omega^{\varepsilon}\right)$. If we have the estimates

$$
\forall \boldsymbol{v} \in V\left(\Omega^{\varepsilon}\right) \quad a_{3 \mathrm{D}}^{\varepsilon}(\boldsymbol{u}-\mathrm{U} \boldsymbol{z}, \boldsymbol{v}) \leq B_{1}^{1 / 2} \mathrm{E}_{3 \mathrm{D}}^{\varepsilon}[\boldsymbol{v}]^{1 / 2},
$$


and

$$
\mathrm{E}_{3 \mathrm{D}}^{\varepsilon}\left[\boldsymbol{u}^{\mathrm{cor}}\right] \leq B_{2}
$$

then there holds

$$
\mathrm{E}_{3 \mathrm{D}}^{\varepsilon}[\boldsymbol{u}-\mathrm{U} \boldsymbol{z}] \leq\left(B_{1}^{1 / 2}+2 B_{2}^{1 / 2}\right)^{2}
$$

Proof. Let $\boldsymbol{u}^{\text {new }}=\mathrm{U} \boldsymbol{z}+\boldsymbol{u}^{\text {cor }} \in V\left(\Omega^{\varepsilon}\right)$. Since $\boldsymbol{u}-\mathrm{U} \boldsymbol{z}=\left(\boldsymbol{u}-\boldsymbol{u}^{\text {new }}\right)+\boldsymbol{u}^{\text {cor }}$, we start from the triangle inequality

$$
\mathrm{E}_{3 \mathrm{D}}^{\varepsilon}[\boldsymbol{u}-\mathrm{U} \boldsymbol{z}]^{1 / 2} \leq \mathrm{E}_{3 \mathrm{D}}^{\varepsilon}\left[\boldsymbol{u}-\boldsymbol{u}^{\text {new }}\right]^{1 / 2}+\mathrm{E}_{3 \mathrm{D}}^{\varepsilon}\left[\boldsymbol{u}^{\mathrm{cor}}\right]^{1 / 2} .
$$

The last term of the rhs is bounded by $B_{2}^{1 / 2}$. As for the first one we write

$$
\begin{aligned}
\mathrm{E}_{3 \mathrm{D}}^{\varepsilon}\left[\boldsymbol{u}-\boldsymbol{u}^{\text {new }}\right] & =a_{3 \mathrm{D}}^{\varepsilon}\left(\boldsymbol{u}-\boldsymbol{u}^{\text {new }}, \boldsymbol{u}-\boldsymbol{u}^{\text {new }}\right) \\
& =a_{3 \mathrm{D}}^{\varepsilon}\left(\boldsymbol{u}-\mathrm{U} \boldsymbol{z}, \boldsymbol{u}-\boldsymbol{u}^{\text {new }}\right)+a_{3 \mathrm{D}}^{\varepsilon}\left(\boldsymbol{u}^{\mathrm{cor}}, \boldsymbol{u}-\boldsymbol{u}^{\text {new }}\right) .
\end{aligned}
$$

Since $\boldsymbol{u}-\boldsymbol{u}^{\text {new }}$ belongs to $V\left(\Omega^{\varepsilon}\right)$, we may use (3.1) and obtain:

$$
\mathrm{E}_{3 \mathrm{D}}^{\varepsilon}\left[\boldsymbol{u}-\boldsymbol{u}^{\text {new }}\right] \leq\left(B_{1}^{1 / 2}+\mathrm{E}_{3 \mathrm{D}}^{\varepsilon}\left[\boldsymbol{u}^{\mathrm{cor}}\right]^{1 / 2}\right) \mathrm{E}_{3 \mathrm{D}}^{\varepsilon}\left[\boldsymbol{u}-\boldsymbol{u}^{\text {new }}\right]^{1 / 2},
$$

whence, using (3.2) again

$$
\mathrm{E}_{3 \mathrm{D}}^{\varepsilon}\left[\boldsymbol{u}-\boldsymbol{u}^{\text {new }}\right]^{1 / 2} \leq B_{1}^{1 / 2}+B_{2}^{1 / 2} .
$$

With (3.4) this gives the estimate (3.3).

Thus, to obtain (2.24), it is sufficient to prove estimates (3.1)-(3.2) with $B_{1}, B_{2} \lesssim$ $A_{S}\left(\varepsilon, \boldsymbol{z}, \boldsymbol{f}^{\mathrm{rem}}\right)$ with

$$
A_{S}\left(\varepsilon, \boldsymbol{z}, \boldsymbol{f}^{\mathrm{rem}}\right)=B_{S}(\varepsilon ; \boldsymbol{z}) \mathrm{E}_{2 \mathrm{D}}^{\varepsilon}[\boldsymbol{z}]+\mathrm{D}^{2} E^{-1}\left\|\boldsymbol{f}^{\mathrm{rem}}\right\|_{\mathrm{L}^{2}\left(\Omega^{\varepsilon}\right)}^{2},
$$

where $B_{S}(\varepsilon ; \boldsymbol{z})$ is defined in (2.24). In $\S 6$, we do this for $B_{1}$ and in $\S 7$ we construct the correction term $\boldsymbol{u}^{\text {cor }}$ and prove that $B_{2} \lesssim A_{S}\left(\varepsilon, \boldsymbol{z}, \boldsymbol{f}^{\mathrm{rem}}\right)$.

\section{FORMAL SERIES REDUCTION}

\section{A SHIFTED DISPLACEMENT AND RECONSTRUCTED DISPLACEMENT}

Let $\boldsymbol{u}$ be the displacement solution of $\left(P_{3 \mathrm{D}}\right)$. We can express this displacement in the shifted normal components introduced by NAGHDI (see [32]) and commonly used in classical shell theory. As a matter of fact, computations are easier when considering the shifted components. The shifter is the tensor $\mu_{\sigma}^{\beta}$ (see [32]) defined by

$$
\mu_{\sigma}^{\beta}\left(x_{\alpha}, x_{3}\right)=\delta_{\sigma}^{\beta}-x_{3} b_{\sigma}^{\beta}\left(x_{\alpha}\right),
$$

where $\delta_{\sigma}^{\beta}$ is the Kronecker tensor. If $\boldsymbol{u}^{\prime}=\left(u_{\sigma}^{\prime}, u_{3}^{\prime}\right)$ is any displacement, the shifted displacement $\boldsymbol{w}^{\prime}=\left(w_{\sigma}^{\prime}, w_{3}^{\prime}\right)$ is defined by the relations

$$
w_{3}^{\prime}=u_{3}^{\prime} \quad \text { and } \quad w_{\sigma}^{\prime}=\left(\mu^{-1}\right)_{\sigma}^{\beta} w_{\beta}^{\prime},
$$


where $\left(\mu^{-1}\right)_{\sigma}^{\beta}$ is the inverse of the shifter. Let $\mathrm{L}$ and $\mathrm{T}$ the interior and boundary operators defined by the relation: For all $\boldsymbol{v} \in V\left(\Omega^{\varepsilon}\right)$ and all $\boldsymbol{u}^{\prime} \in \mathrm{H}^{1}\left(\Omega^{\varepsilon}\right)^{3}$

$$
a_{3 \mathrm{D}}\left(\boldsymbol{u}^{\prime}, \boldsymbol{v}\right)=-\left\langle\mathrm{L} \boldsymbol{w}^{\prime}, \boldsymbol{v}\right\rangle_{\mathrm{L}^{2}\left(\Omega^{\varepsilon}\right)}-\left\langle\mathrm{T} \boldsymbol{w}^{\prime}, \boldsymbol{v}\right\rangle_{\Gamma_{ \pm}^{\varepsilon}}
$$

The coefficients of the operators $\mathrm{L}$ and $\mathrm{T}$ express in terms of the normal coordinate $x_{3}$, the covariant derivative $\mathrm{D}_{\alpha}$ and the curvature tensor $b_{\alpha \beta}$, see [19]. If we denote in particular by $\boldsymbol{w}=\left(w_{\sigma}, w_{3}\right)$ the shifted displacement associated with $\boldsymbol{u}$, we find that $\boldsymbol{w}$ is solution of the boundary value problem

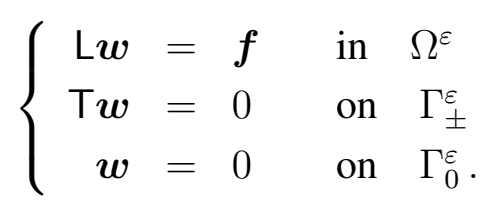

Let $\boldsymbol{z}=\left(z_{\sigma}, z_{3}\right)$ be solution of $\left(P_{2 \mathrm{D}}\right)$. With the three-dimensional displacement $\mathbf{U} \boldsymbol{z}$ defined in (2.23) is associated the three-dimensional shifted displacement $\mathrm{W} z$ given by the formula

$$
\mathbf{W} \boldsymbol{z}=\left\{\begin{array}{l}
z_{\sigma}-x_{3} \theta_{\sigma}(\boldsymbol{z}) \\
z_{3}-p x_{3} \gamma_{\alpha}^{\alpha}(\boldsymbol{z})+p \frac{x_{3}^{2}}{2} \rho_{\alpha}^{\alpha}(\boldsymbol{z}) .
\end{array}\right.
$$

\section{B SCALING IN THE 3D bOUNDARY VALUE PROBLEM}

The formal series approach of $[19,18]$ relies on the scaling $X_{3}=\varepsilon^{-1} x_{3}$ which transforms problem (4.2) into a problem posed on a domain independent of $\varepsilon$, with operators which are power series of $\varepsilon$. This allows a formal series reduction of the 3D problem.

The scaling $x_{3} \mapsto X_{3}=\varepsilon^{-1} x_{3}$ is one-to-one from the shell $\Omega^{\varepsilon}$ onto the manifold $\mathcal{Q}:=S \times(-1,1)$ and we denote by $\Gamma_{ \pm}$its upper and lower faces $S \times\{ \pm 1\}$ and by $\Gamma_{0}$ its lateral boundary $\partial S \times(-1,1)$. Likewise $V(\mathcal{Q})$ denotes the space of $\boldsymbol{v} \in H^{1}(\mathcal{Q})^{3}$ which satisfies the Dirichlet boundary condition $\left.\boldsymbol{v}\right|_{\Gamma_{0}}=0$.

In the following, we denote by $\underline{\boldsymbol{u}}$ the displacement $\boldsymbol{u}$ viewed on the manifold $\mathcal{Q}$. In a local coordinate system $\left(x_{\alpha}\right)$ on $S$, this means that $\underline{\boldsymbol{u}}\left(x_{\alpha}, X_{3}\right)=\boldsymbol{u}\left(x_{\alpha}, x_{3}\right)$ for $X_{3}=$ $\varepsilon^{-1} x_{3}$. Similarly, $\underline{\boldsymbol{w}}$ and $\underline{\boldsymbol{f}}$ correspond to the shifted displacement $\boldsymbol{w}$ and the loading forces $\boldsymbol{f}$. To denote the displacements $\mathrm{U} z$ and $\mathrm{W} \boldsymbol{z}$ on $\mathcal{Q}$, we use the notations $\mathrm{U}(\varepsilon) \boldsymbol{z}$ and $\mathrm{W}(\varepsilon) z$ so that we have with (4.3)

$$
\mathrm{W}(\varepsilon) \boldsymbol{z}=\left\{\begin{array}{l}
z_{\sigma}-\varepsilon X_{3} \theta_{\sigma}(\boldsymbol{z}), \\
z_{3}-\varepsilon X_{3} p \gamma_{\alpha}^{\alpha}(\boldsymbol{z})+\varepsilon^{2} \frac{X_{3}^{2}}{2} p \rho_{\alpha}^{\alpha}(\boldsymbol{z}),
\end{array}\right.
$$

and a similar formula for $\mathrm{U}(\varepsilon) \boldsymbol{z}$.

In the same way, we define the three-dimensional energy on $\mathcal{Q}$ by the formula

$$
\mathrm{E}_{3 \mathrm{D}}(\varepsilon)[\underline{\boldsymbol{u}}]=\mathrm{E}_{3 \mathrm{D}}^{\varepsilon}[\boldsymbol{u}]
$$

involving the scaled strain tensor $e_{i j}(\varepsilon)(\underline{\boldsymbol{u}})=e_{i j}(\boldsymbol{u})$, and associated with the bilinear form $a_{3 \mathrm{D}}(\varepsilon)(\cdot, \cdot)$ on $V(\mathcal{Q})$. In particular, we will often use the relation, for $\boldsymbol{v} \in \mathrm{H}^{1}(\mathcal{Q})$,

$$
\left\|e_{i j}(\varepsilon)(\boldsymbol{v})\right\|_{\mathrm{L}^{2}(\mathcal{Q})}^{2} \simeq \varepsilon^{-1} E^{-1} \mathrm{E}_{3 \mathrm{D}}(\varepsilon)[\boldsymbol{v}] .
$$


Note that with these notations, Korn inequalities (2.20) read, for $\boldsymbol{v} \in V(\mathcal{Q})$,

$$
\begin{aligned}
\|\boldsymbol{v}\|_{\mathrm{L}^{2}(\mathcal{Q})}^{2} & \leq \mathrm{D}^{4} E^{-1} \varepsilon^{-3} \mathrm{E}_{3 \mathrm{D}}(\varepsilon)[\boldsymbol{v}] \\
\left\|\mathrm{D}_{\alpha} \boldsymbol{v}\right\|_{\mathrm{L}^{2}(\mathcal{Q})}^{2} & \leq \mathrm{D}^{2} E^{-1} \varepsilon^{-3} \mathrm{E}_{3 \mathrm{D}}(\varepsilon)[\boldsymbol{v}] \\
\left\|\partial_{X_{3}} \boldsymbol{v}\right\|_{\mathrm{L}^{2}(\mathcal{Q})}^{2} & \leq \mathrm{D}^{2} E^{-1} \varepsilon^{-1} \mathrm{E}_{3 \mathrm{D}}(\varepsilon)[\boldsymbol{v}]
\end{aligned}
$$

The scaled displacement $\underline{\boldsymbol{u}} \in V(\mathcal{Q})$ is solution of the variational problem

$$
\forall \boldsymbol{v} \in V(\mathcal{Q}), \quad a_{3 \mathrm{D}}(\varepsilon)(\underline{\boldsymbol{u}}, \boldsymbol{v})=\varepsilon\langle\underline{\boldsymbol{f}}, \boldsymbol{v}\rangle_{\mathcal{Q} ; \varepsilon},
$$

where for $\boldsymbol{v}$ and $\boldsymbol{v}^{\prime}$ in $\mathrm{L}^{2}(\mathcal{Q})^{3}$ (see (2.2))

$$
\left\langle\boldsymbol{v}, \boldsymbol{v}^{\prime}\right\rangle_{\mathcal{Q} ; \varepsilon}=\int_{\mathcal{Q}}\left(\boldsymbol{v} \cdot \boldsymbol{v}^{\prime}\right)\left(1+h\left(\varepsilon X_{3}\right)\right) \mathrm{d} S \mathrm{~d} X_{3}
$$

The 3D interior operator $\mathrm{L}\left(x_{\alpha}, x_{3} ; \mathrm{D}_{\alpha}, \partial_{3}\right)$ in problem (4.2) is transformed into the operator $\mathrm{L}(\varepsilon)$

$$
\mathrm{L}(\varepsilon)\left(x_{\alpha}, X_{3} ; \mathrm{D}_{\alpha}, \partial_{X_{3}}\right):=\mathrm{L}\left(x_{\alpha}, \varepsilon X_{3} ; \mathrm{D}_{\alpha}, \varepsilon^{-1} \partial_{X_{3}}\right),
$$

and similarly for the boundary operators $\mathrm{T}\left(x_{\alpha}, x_{3} ; \mathrm{D}_{\alpha}, \partial_{3}\right)$ and $\mathrm{T}(\varepsilon)$. Note that on the manifold $\mathcal{Q}$, the variable $X_{3}$ and the partial derivative $\partial_{X_{3}}$ are adimensional. The operators $\mathrm{L}(\varepsilon)$ and $\mathrm{T}(\varepsilon)$ expand in power series of $\varepsilon$ :

$$
\mathrm{L}(\varepsilon)=\varepsilon^{-2} \sum_{k=0}^{\infty} \varepsilon^{k} \mathrm{~L}^{k} \quad \text { and } \quad \mathrm{T}(\varepsilon)=\varepsilon^{-1} \sum_{k=0}^{\infty} \varepsilon^{k} \mathrm{~T}^{k},
$$

where $\mathrm{L}^{k}$ and $\mathrm{T}^{k}$ are intrinsic operators in $\mathcal{Q}$ which are polynomial in $X_{3}$ and in $\partial_{X_{3}}$ with coefficients $\boldsymbol{b}$-homogeneous operators of degree $k$, see [19, Thm. 3.3].

So problem (4.2) is equivalent to the problem

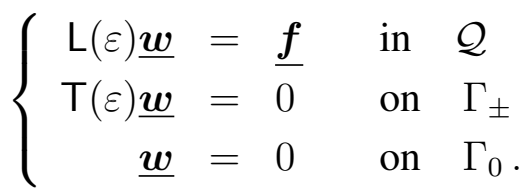

Moreover there holds for all $\boldsymbol{v} \in V(\mathcal{Q})$ and all $\boldsymbol{u}^{\prime} \in \mathrm{H}^{1}(\mathcal{Q})^{3}$ (see (4.1))

$$
a_{3 \mathrm{D}}(\varepsilon)\left(\boldsymbol{u}^{\prime}, \boldsymbol{v}\right)=-\varepsilon\left\langle\mathrm{L}(\varepsilon) \boldsymbol{w}^{\prime}, \boldsymbol{v}\right\rangle_{\mathcal{Q} ; \varepsilon}-\varepsilon\left\langle\mathrm{T}(\varepsilon) \boldsymbol{w}^{\prime}, \boldsymbol{v}\right\rangle_{\Gamma_{ \pm} ; \varepsilon}
$$

where

$$
\left\langle\boldsymbol{v}, \boldsymbol{v}^{\prime}\right\rangle_{\Gamma_{ \pm} ; \varepsilon}=\int_{\mathcal{Q}}\left(\boldsymbol{v} \cdot \boldsymbol{v}^{\prime}\right)\left(1+h\left( \pm \varepsilon X_{3}\right)\right) \mathrm{d} S .
$$

Here, $\boldsymbol{w}^{\prime}$ is the scaled shifted displacement corresponding to $\boldsymbol{u}^{\prime}$ i.e.

$$
u_{3}^{\prime}=w_{3}^{\prime} \quad \text { and } \quad u_{\sigma}^{\prime}=\left(\delta_{\sigma}^{\beta}-\varepsilon X_{3} b_{\sigma}^{\beta}\right) w_{\beta}^{\prime} .
$$

For instance (4.11) holds with $\boldsymbol{u}^{\prime}=\mathrm{U}(\varepsilon) \boldsymbol{z}$ and $\boldsymbol{w}^{\prime}=\mathrm{W}(\varepsilon) \boldsymbol{z}$. 


\section{C Solution OF TRANSVERSE PROBlemS}

The treatment of the first two equations of (4.10) can be performed by solving Neumann problems in $X_{3}$ and introducing suitable compatibility conditions. This can be done in a fully exact way without approximation using the formalism of formal Laurent series and formal power series, as follows.

With expansions (4.9) we associate the formal Laurent series

$$
\mathrm{L}(\mathrm{X})=\sum_{k \geq-2} \mathrm{~L}^{k+2} \mathrm{X}^{k} \quad \text { and } \mathrm{T}(\mathrm{X})=\sum_{k \geq-1} \mathrm{~T}^{k+1} \mathrm{X}^{k}
$$

The three-dimensional formal series system $\{L(X), T(X)\}$ can be reduced to a two dimensional one, cf. [19, Thm. 4.1]:

Theorem 4.1 There exist two unique formal power series $\mathrm{V}(\mathrm{X})=\sum_{k \geq 0} \mathrm{~V}^{k} \mathrm{X}^{k}$ and $\mathrm{A}(\mathrm{X})=$ $\sum_{k \geq 0} \mathrm{~A}^{k} \mathrm{X}^{k}$ satisfying the following three conditions:

1. The coefficients $\mathrm{V}^{k}$ are reconstruction operators acting from $\Sigma(S)$ with values in $\mathscr{C}^{\infty}(I, \Sigma(S))$, and such that for all $\boldsymbol{z} \in \Sigma(S)$

$$
\bigvee^{0} \boldsymbol{z}=\boldsymbol{z} \text { and } \forall k \geq 1, \bigvee^{k} \boldsymbol{z}=0 \text { on } S,
$$

2. The coefficients $\mathrm{A}^{k}$ are $2 D$ operators acting from $\Sigma(S)$ into itself,

3. There holds the formal series equation

$$
\begin{cases}\mathrm{L}(\mathrm{X}) \mathrm{V}(\mathrm{X})=-\mathcal{I} \circ \mathrm{A}(\mathrm{X}) & \text { in } \mathcal{Q} \\ \mathrm{T}(\mathrm{X}) \mathrm{V}(\mathrm{X})=0 & \text { on } \Gamma_{ \pm} .\end{cases}
$$

Here, the operator $\mathcal{I}$ is the natural embedding operator from $\Sigma(S)$ to the space $\mathscr{C}^{\infty}(I, \Sigma(S))$ and the product between two formal series is the standard Cauchy product.

Thus the equation (4.13) means that there holds

$$
\begin{gathered}
\mathrm{L}^{0} \mathrm{~V}^{0}=0, \quad \mathrm{~L}^{0} \mathrm{~V}^{1}+\mathrm{L}^{1} \mathrm{~V}^{0}=0, \quad \sum_{j+k=i} \mathrm{~L}^{j} \mathrm{~V}^{k}=-\mathcal{I} \circ \mathrm{A}^{i-2}, \quad i \geq 2 \\
\sum_{j+k=i} \mathrm{~T}^{j} \mathrm{~V}^{k}=0, \quad i \geq 0 .
\end{gathered}
$$

Following the proof of [19, Thm. 4.1], we can see that the term $\mathrm{V}^{k}$ of the formal series $\mathrm{V}(\mathrm{X})$ is polynomial in $X_{3}$ and $\boldsymbol{b}$-homogeneous of degree $k$. We have

$$
\mathrm{V}_{\sigma}^{1} \boldsymbol{z}=-X_{3} \theta_{\sigma}(\boldsymbol{z}), \quad \mathrm{V}_{3}^{1} \boldsymbol{z}=-p X_{3} \gamma_{\alpha}^{\alpha}(\boldsymbol{z})
$$

and

$$
\mathrm{V}_{\sigma}^{2} \boldsymbol{z}=\frac{X_{3}^{2}}{2} p \mathrm{D}_{\sigma} \gamma_{\alpha}^{\alpha}(\boldsymbol{z}), \quad \mathrm{V}_{3}^{2} \boldsymbol{z}=p \frac{X_{3}^{2}}{2}\left(\rho_{\alpha}^{\alpha}(\boldsymbol{z})-p b_{\alpha}^{\alpha} \gamma_{\beta}^{\beta}(\boldsymbol{z})-2 b_{\alpha}^{\beta} \gamma_{\beta}^{\alpha}(\boldsymbol{z})\right)
$$


as first terms. Actually, the reconstruction operator $W(\varepsilon): z \rightarrow W(\varepsilon) z$ in (4.4) coincides with $\mathrm{V}^{0}+\varepsilon \mathrm{V}^{1}+\varepsilon^{2}\left(\mathrm{~V}^{2}-\mathrm{v}^{2}\right)$ where $\mathrm{v}^{2}$ is a residual part of the operator $\mathrm{V}^{2}$.

The term $\mathrm{A}^{k}$ of the formal series $\mathrm{A}(\mathrm{X})$ is a $\boldsymbol{b}$-homogeneous operator of degree $k+2$. The zero-th order term $A^{0}$ coincides with the membrane operator $M, A^{1}$ is zero, so that $A(\varepsilon)=M+\varepsilon^{2} A^{2}+\cdots$. Moreover, adapting the proof of [19, Prop. 4.5] we obtain the following estimate for the difference $A^{2}-B$ where $B$ is the Koiter bending operator: If $\boldsymbol{z}$ and $\boldsymbol{\eta} \in \Sigma(S)$ and $\boldsymbol{\eta}$ satisfies the boundary condition $\left.\boldsymbol{\eta}\right|_{\partial S}=0$,

$$
\begin{aligned}
\left|\left\langle\left(\mathrm{A}^{2}-\mathrm{B}\right) \boldsymbol{z}, \boldsymbol{\eta}\right\rangle_{\mathrm{L}^{2}(S)}\right| \lesssim E\left(|\boldsymbol{\gamma}(\boldsymbol{z})|_{2 ; S}|\boldsymbol{\gamma}(\boldsymbol{\eta})|_{0 ; S}+\kappa_{2}^{2}|\boldsymbol{z}|_{1 ; S}^{(\boldsymbol{b})}|\boldsymbol{\gamma}(\boldsymbol{\eta})|_{0 ; S}\right. \\
\left.\quad+\kappa_{1}\left|z_{3}\right|_{2 ; S}|\boldsymbol{\gamma}(\boldsymbol{\eta})|_{0 ; S}+\kappa_{1}|\boldsymbol{\gamma}(\boldsymbol{z})|_{1 ; S}|\boldsymbol{\eta}|_{1 ; S}^{(\boldsymbol{b})}+\kappa_{2}^{2}|\boldsymbol{\gamma}(\boldsymbol{z})|_{0 ; S}|\boldsymbol{\eta}|_{1 ; S}^{(\boldsymbol{b})}\right),
\end{aligned}
$$

where the constants $\kappa_{j}, j=1,2$ are defined in (2.15).

\section{A PRIORI ESTIMATES}

Let $\boldsymbol{z}=\left(z_{\sigma}, z_{3}\right)$ where $\left(z_{\sigma}\right)$ is a 1 -form field on $S$ and $z_{3}$ a function on $S$. In this section we prove estimates for the Sobolev norms of $z$, first by Sobolev norms of its strain tensors $\gamma:=\gamma(\boldsymbol{z})$ and $\boldsymbol{\rho}:=\boldsymbol{\rho}(\boldsymbol{z})$ and then, with the help of the wave lengths $L_{k}$, by its quadratic energy $\mathrm{E}_{2 \mathrm{D}}^{\varepsilon}[\boldsymbol{z}]$, cf. (2.10).

Lemma 5.1 There exists a positive adimensional constant $A$ such that

$$
\begin{array}{ll}
\forall \boldsymbol{z} \in \mathbf{H}^{2} \times \mathrm{H}^{1}(S), \quad\left|z_{\sigma}\right|_{2 ; S} \leq A\left(|\boldsymbol{\gamma}|_{1 ; S}+\kappa_{1}|\boldsymbol{z}|_{1 ; S}+\kappa_{2}^{2}|\boldsymbol{z}|_{0 ; S}\right), \\
\forall \boldsymbol{z} \in \mathbf{H}^{1} \times \mathrm{H}^{2}(S), \quad\left|z_{3}\right|_{2 ; S} \leq A\left(|\boldsymbol{\rho}|_{0 ; S}+\kappa_{1}|\boldsymbol{z}|_{1 ; S}+\kappa_{2}^{2}|\boldsymbol{z}|_{0 ; S}\right) .
\end{array}
$$

Proof. Let us recall (see e.g. $[32,18]$ ) that we have the following relation for the commutation of two covariant derivatives: For all $z_{\alpha}$,

$$
\mathrm{D}_{\alpha} \mathrm{D}_{\beta} z_{\sigma}-\mathrm{D}_{\beta} \mathrm{D}_{\alpha} z_{\sigma}=R_{\beta \alpha \sigma \nu} z^{\nu}
$$

where the Riemann tensor $R_{\beta \alpha \sigma \nu}$ of $S$ is given by

$$
R_{\beta \alpha \sigma \nu}=b_{\beta \nu} b_{\alpha \sigma}-b_{\alpha \nu} b_{\beta \sigma} .
$$

Using this relation, and setting $\bar{\gamma}_{\alpha \sigma}=\frac{1}{2}\left(\mathrm{D}_{\alpha} z_{\sigma}+\mathrm{D}_{\sigma} z_{\alpha}\right)$, we have

$$
\mathrm{D}_{\alpha} \mathrm{D}_{\beta} z_{\sigma}=\mathrm{D}_{\alpha} \bar{\gamma}_{\beta \sigma}-\mathrm{D}_{\sigma} \bar{\gamma}_{\alpha \beta}+\mathrm{D}_{\beta} \bar{\gamma}_{\sigma \alpha}-\frac{1}{2} R_{\beta \alpha \sigma \nu} z^{\nu}+\frac{1}{2} R_{\sigma \alpha \beta \nu} z^{\nu}+\frac{1}{2} R_{\sigma \beta \alpha \nu} z^{\nu} .
$$

This formula clearly implies that there exists an adimensional constant $A$ such that

$$
\left|z_{\sigma}\right|_{2 ; S} \leq A\left(|\bar{\gamma}|_{1 ; S}+\kappa_{1}^{2}|\boldsymbol{z}|_{0 ; S}\right) .
$$

As $\gamma_{\alpha \beta}=\bar{\gamma}_{\alpha \beta}-b_{\alpha \beta} z_{3}$, this finally gives (5.1a).

The estimate (5.1b) is an easy consequence of the expression (2.8) of $\rho_{\alpha \beta}$. 
From the previous estimates, we are going to deduce bounds for $\left|z_{\sigma}\right|_{n ; S}^{(\boldsymbol{b})}$ and $\left|z_{3}\right|_{n ; S}^{(\boldsymbol{b})}$ by induction over $n$. In the remaining part of this section, we use the following notation: $f \lesssim g$ means that there exist an adimensional constant $A$ such that $f \leq A g$. When $f \lesssim g$ and $g \lesssim f$ we write $f \simeq g$.

Applying the estimates (5.1b) and (5.1a) to $\mathrm{D}_{\delta} \boldsymbol{z}$ in combination with (5.2) and then using induction, we find the estimates for any $n \geq 2$ :

$$
\begin{aligned}
&\left|z_{\sigma}\right|_{n ; S} \lesssim|\gamma|_{n-1 ; S}+\sum_{1 \leq j \leq n} \kappa_{j}^{j}|\boldsymbol{z}|_{n-j ; S} \\
&\left|z_{3}\right|_{n ; S} \lesssim|\boldsymbol{\rho}|_{n-2 ; S}+\sum_{1 \leq j \leq n} \kappa_{j}^{j}|\boldsymbol{z}|_{n-j ; S} .
\end{aligned}
$$

Combining (5.3a) and (5.3b) for $n, n-1, \ldots, 0$ we obtain for all $n \geq 2$ and all $\boldsymbol{z} \in$ $\mathbf{H}^{n} \times \mathrm{H}^{n}(S)^{3}$

$$
\begin{aligned}
\left|z_{\sigma}\right|_{n ; S}^{(\boldsymbol{b})} & \lesssim|\boldsymbol{\gamma}|_{n-1 ; S}^{(\boldsymbol{b})}+\sum_{1 \leq j \leq n-2} \kappa_{j}^{j}|\boldsymbol{\rho}|_{n-2-j ; S}+\kappa_{n-1}^{n-1}|\boldsymbol{z}|_{1 ; S}+\kappa_{n}^{n}|\boldsymbol{z}|_{0 ; S} \\
\left|z_{3}\right|_{n ; S}^{(\boldsymbol{b})} & \lesssim|\boldsymbol{\rho}|_{n-2 ; S}^{(\boldsymbol{b})}+\sum_{1 \leq j \leq n-1} \kappa_{j}^{j}|\boldsymbol{\gamma}|_{n-1-j ; S}+\kappa_{n-1}^{n-1}|\boldsymbol{z}|_{1 ; S}+\kappa_{n}^{n}|\boldsymbol{z}|_{0 ; S}
\end{aligned}
$$

We can eliminate the terms $\kappa_{n-1}^{n-1}|\boldsymbol{z}|_{1 ; S}+\kappa_{n}^{n}|\boldsymbol{z}|_{0 ; S}$ with Poincaré type estimates, see [6]. Indeed, we can prove that for a given $n \geq 1$ there exist an adimensional constant $A_{n}$ such that

$$
\forall \boldsymbol{z} \in \mathbf{H}_{0}^{1} \times \mathrm{H}_{0}^{2}(S), \quad \kappa_{n}|\boldsymbol{z}|_{1 ; S}+\kappa_{n}^{2}|\boldsymbol{z}|_{0 ; S} \leq A_{n}\left(|\boldsymbol{\rho}|_{0 ; S}+\kappa_{n}|\boldsymbol{\gamma}|_{0 ; S}\right)
$$

Combining this with (5.4a)-(5.4b) we obtain

$$
\begin{aligned}
\left|z_{\sigma}\right|_{n ; S}^{(\boldsymbol{b})} & \lesssim|\boldsymbol{\gamma}|_{n-1 ; S}^{(\boldsymbol{b})}+\kappa_{n} \sum_{0 \leq j \leq n-3} \kappa_{n}^{j}|\boldsymbol{\rho}|_{n-3-j ; S} \\
\left|z_{3}\right|_{n ; S}^{(\boldsymbol{b})} & \lesssim|\boldsymbol{\rho}|_{n-2 ; S}^{(\boldsymbol{b})}+\kappa_{n} \sum_{0 \leq j \leq n-2} \kappa_{n}^{j}|\boldsymbol{\gamma}|_{n-2-j ; S} .
\end{aligned}
$$

Then we use the definition (2.17) of the wave lengths together with (2.18), and deduce from the previous inequalities that there exists an adimensional constant $A_{n}$ such that

$$
|\boldsymbol{z}|_{n ; S}^{(\boldsymbol{b})} \leq A\left(|\boldsymbol{\gamma}|_{0 ; S} \sum_{j+k=n-1} L_{n-1}^{-k} \kappa_{n}^{j}+|\boldsymbol{\rho}|_{0 ; S} \sum_{j+k=n-2} L_{n-1}^{-k} \kappa_{n}^{j}\right) \text {. }
$$

Remark 5.2 The adimensional constants $A$ in (5.7) depend on $n$ and on $S$ via the Poincaré type estimates (5.5). We can prove that these constants remain bounded in a family $\left\{S_{\delta}\right\}$ of shallow shells in the sense of [13,9], see also [3], as $\delta \rightarrow 0$.

Using relations (2.22) we find immediately that $\mathrm{E}_{2 \mathrm{D}}^{\varepsilon}[\boldsymbol{z}] \simeq E \varepsilon\left(|\gamma|_{0 ; S}^{2}+\varepsilon^{2}|\boldsymbol{\rho}|_{0 ; S}^{2}\right)$. Hence

$$
|\gamma|_{0 ; S}^{2} \lesssim A E^{-1} \varepsilon^{-1} \mathrm{E}_{2 \mathrm{D}}^{\varepsilon}[\boldsymbol{z}] \quad \text { and } \quad|\boldsymbol{\rho}|_{0 ; S}^{2} \lesssim A E^{-1} \varepsilon^{-3} \mathrm{E}_{2 \mathrm{D}}^{\varepsilon}[\boldsymbol{z}]
$$

Estimate (5.7) combined with (5.8) yields the following energy estimates for any two-dimensional displacement $\boldsymbol{z}$ satisfying the conditions of the clamped boundary: 
Theorem 5.3 For any $n \geq 2$, there exists an adimensional constant $A>0$ so that for any $\boldsymbol{z} \in \mathbf{H}_{0}^{1} \times \mathrm{H}_{0}^{2}(S)$ satisfying $\gamma \in \mathrm{H}^{n-1}(S)$ and $\boldsymbol{\rho} \in \mathrm{H}^{n-2}(S)$, there holds for any $\varepsilon>0$ :

$$
\left(|\boldsymbol{z}|_{n ; S}^{(\boldsymbol{b})}\right)^{2} \leq \frac{A E^{-1}}{\varepsilon^{3}}\left(\sum_{i, j, k \in G_{n}} \frac{\varepsilon^{2 i} \kappa_{n}^{2 j}}{L_{n-1}^{2 k}}\right) \mathrm{E}_{2 \mathrm{D}}^{\varepsilon}[\boldsymbol{z}]
$$

where $E$ is the Young modulus, $\kappa_{n}$ the $n$-th constant estimating the curvature, $L_{n-1}$ is the global wave length of $\boldsymbol{z}$, cf. Definitions 2.1 and 2.4, and where

$$
G_{n}=\left\{(i, j, k) \in \mathbb{N}^{3} \mid i \in\{0,1\}, j+k=i+n-2\right\}
$$

\section{INNER ESTIMATE}

In this section, we prove the following result:

Proposition 6.1 With the definitions of Section 2, let $\kappa=\kappa_{5}, r=1 / \kappa$ and $L=L_{4}$. For $v \in V(\mathcal{Q})$, we have the estimate

$$
a_{3 \mathrm{D}}(\varepsilon)(\underline{\boldsymbol{u}}-\mathrm{U}(\varepsilon) \boldsymbol{z}, \boldsymbol{v}) \lesssim B_{1}^{1 / 2} \mathrm{E}_{3 \mathrm{D}}(\varepsilon)[\boldsymbol{v}]^{1 / 2}
$$

where

$$
B_{1}=\mathrm{D}^{2} E^{-1} \varepsilon\left\|\underline{\boldsymbol{f}}^{\mathrm{rem}}\right\|_{\mathcal{Q} ; \varepsilon}^{2}+B_{S}^{1}(\varepsilon ; \boldsymbol{z}) \mathrm{E}_{2 \mathrm{D}}^{\varepsilon}[\boldsymbol{z}],
$$

where $\underline{f}^{\mathrm{rem}}=\underline{\boldsymbol{f}}-\boldsymbol{g}$ and

$$
B_{S}^{1}(\varepsilon ; \boldsymbol{z})=\left(1+\frac{\mathrm{D}^{4}}{\mathrm{r}^{4}}\right)\left(\sum_{F} \frac{\varepsilon^{2 k}}{L^{2 i} \mathrm{r}^{2 j}}+\frac{\varepsilon^{4} \mathrm{D}^{2}}{L^{6}}+\frac{\varepsilon^{6} \mathrm{D}^{2}}{L^{8}}\right)
$$

where $F$ is the finite set $\left\{(i, j, k) \in \mathbb{N}^{3} \mid i+j=k, k \in\{1,2,3,4\}\right\}$.

Scaling back to $\Omega^{\varepsilon}$, the previous result implies that for $\boldsymbol{v} \in V\left(\Omega^{\varepsilon}\right)$, we have

$$
a_{3 \mathrm{D}}^{\varepsilon}(\boldsymbol{u}-\mathrm{U} \boldsymbol{z}, \boldsymbol{v}) \lesssim B_{1}^{1 / 2} \mathrm{E}_{3 \mathrm{D}}^{\varepsilon}[\boldsymbol{v}]^{1 / 2}
$$

where $B_{1}$ is given by (6.1). Note that using (4.8) we have $\varepsilon\left\|\underline{f}^{\text {rem }}\right\|_{\mathcal{Q} ; \varepsilon}^{2}=\| \boldsymbol{f}^{\text {rem } \|_{L^{2}\left(\Omega^{\varepsilon}\right)}^{2}}$.

Before starting the proof of the proposition, let us prove that $B_{1}$ in (6.1) satisfies $B_{1} \lesssim$ $A_{S}\left(\varepsilon, \boldsymbol{z}, \boldsymbol{f}^{\text {rem }}\right)$ given by (3.5) and (2.24) under the hypothesis $\varepsilon<r$ and $\varepsilon^{2} / L^{2} \leq M$ of Theorem 2.8.

First, the ratio $\mathrm{D} / \mathrm{r}$ is an adimensional constant depending on $S$ only. Hence,

$$
B_{S}^{1}(\varepsilon ; \boldsymbol{z}) \lesssim \sum_{F} \frac{\varepsilon^{2 k}}{L^{2 i} r^{2 j}}+\frac{\varepsilon^{4} \mathrm{D}^{2}}{L^{6}}+\frac{\varepsilon^{6} \mathrm{D}^{2}}{L^{8}}
$$

Now it is clear that under the hypothesis $\varepsilon^{2} / L^{2} \leq M$ we have

$$
\sum_{F} \frac{\varepsilon^{2 k}}{L^{2 i} r^{2 j}} \lesssim\left(\frac{\varepsilon^{2}}{r^{2}}+\frac{\varepsilon^{2}}{L^{2}}\right)\left(1+M^{3}\right) \quad \text { and } \quad \frac{\varepsilon^{6} \mathrm{D}^{2}}{L^{8}} \leq M \frac{\varepsilon^{4} \mathrm{D}^{2}}{L^{6}}
$$


and this proves that $B_{1} \lesssim A_{S}\left(\varepsilon, \boldsymbol{z}, \boldsymbol{f}^{\mathrm{rem}}\right)$ with an adimensional constant satisfying (2.25).

The leading idea of the proof of Proposition 6.1 is to replace $U(\varepsilon) z$ with a more precise reconstructed displacement $U^{\text {asy }}(\varepsilon) z$ : Working in shifted displacement, we define the new reconstruction operator $W^{\text {asy }}(\varepsilon)$ as the first five terms of the formal series $V(X)$ introduced in Theorem 4.1:

$$
\mathrm{W}^{\text {asy }}(\varepsilon)=\mathrm{V}^{0}+\varepsilon \mathrm{V}^{1}+\varepsilon^{2} \mathrm{~V}^{2}+\varepsilon^{3} \mathrm{~V}^{3}+\varepsilon^{4} \mathrm{~V}^{4}
$$

To this operator corresponds the operator $U^{\text {asy }}(\varepsilon)$ as in (4.12).

We first prove the following lemma:

Lemma 6.2 For $\boldsymbol{v} \in V(\mathcal{Q})$, we have the estimate

$$
a_{3 \mathrm{D}}(\varepsilon)\left(\underline{\boldsymbol{u}}-\mathrm{U}^{\text {asy }}(\varepsilon) \boldsymbol{z}, \boldsymbol{v}\right) \lesssim B_{1}^{1 / 2} \mathrm{E}_{3 \mathrm{D}}(\varepsilon)[\boldsymbol{v}]^{1 / 2}
$$

where $\mathrm{U}^{\text {asy }}(\varepsilon) \boldsymbol{z}$ is given in shifted components by the displacement $\mathrm{W}^{\text {asy }}(\varepsilon) \boldsymbol{z}$ defined in (6.3) and $B_{1}$ by (6.1).

Remark 6.3 Owing to (2.2), it is clear that the norms $\|\cdot\|_{\mathcal{Q} ; \varepsilon}$ and $\|\cdot\|_{\mathrm{L}^{2}(\mathcal{Q})}$ are equivalent up to adimensional constants.

Proof of Lemma 6.2. Let $\boldsymbol{v} \in V(\mathcal{Q})$. We split $a_{3 \mathrm{D}}(\varepsilon)\left(\underline{\boldsymbol{u}}-\mathrm{U}^{\text {asy }}(\varepsilon) \boldsymbol{z}, \boldsymbol{v}\right)$ into two terms. Since $\boldsymbol{u}$ is solution of $\left(P_{3 \mathrm{D}}\right)$, eq. (4.7) yields

$$
a_{3 \mathrm{D}}(\varepsilon)(\underline{\boldsymbol{u}}, \boldsymbol{v})=\varepsilon\langle\underline{\boldsymbol{f}}, \boldsymbol{v}\rangle_{\mathcal{Q} ; \varepsilon} .
$$

For the second term, using (4.11) we obtain

$$
a_{3 \mathrm{D}}(\varepsilon)\left(\mathrm{U}^{\text {asy }}(\varepsilon) \boldsymbol{z}, \boldsymbol{v}\right)=-\varepsilon\left\langle\mathrm{L}(\varepsilon) \mathrm{W}^{\text {asy }}(\varepsilon) \boldsymbol{z}, \boldsymbol{v}\right\rangle_{\mathcal{Q} ; \varepsilon}-\varepsilon\left\langle\mathrm{T}(\varepsilon) \mathrm{W}^{\text {asy }}(\varepsilon) \boldsymbol{z}, \boldsymbol{v}\right\rangle_{\Gamma_{ \pm} ; \varepsilon} .
$$

By definition of $W^{\text {asy }}(\varepsilon)$, and using (4.14a), we find

$$
\begin{aligned}
-\mathrm{L}(\varepsilon) \mathrm{W}^{\text {asy }}(\varepsilon)= & \mathrm{M}+\varepsilon^{2} \mathrm{~A}^{2}+ \\
& +\varepsilon^{3}\left(\mathrm{~L}^{1} \mathrm{~V}^{4}+\mathrm{L}^{2} \mathrm{~V}^{3}+\mathrm{L}^{3} \mathrm{~V}^{2}+\mathrm{L}^{4} \mathrm{~V}^{1}+\mathrm{L}^{5} \mathrm{~V}^{0}\right)+\varepsilon^{4} \sum_{0 \leq i \leq 4} \overline{\mathrm{L}}^{i}(\varepsilon) \mathrm{V}^{i},
\end{aligned}
$$

where the operators $\bar{L}^{i}(\varepsilon)$ are given by the convergent power series

$$
\overline{\mathrm{L}}^{i}(\varepsilon)=\sum_{k=0}^{\infty} \varepsilon^{k} \mathrm{~L}^{k+6-i}
$$

and define operators of order 2 in $\mathrm{D}_{\alpha}$. The convergence of these series rely on the uniform estimates for all $n \geq 3$ (see [19, Thm. 3.3])

$$
\begin{aligned}
\left\|\mathrm{L}^{n} \boldsymbol{v}\right\|_{\mathrm{L}^{2}(\mathcal{Q})} \lesssim n E\left(\kappa_{1}^{n-2}\left\|\mathrm{D}_{[2]} \boldsymbol{v}\right\|_{\mathrm{L}^{2}(\mathcal{Q})}\right. & +\kappa_{2}^{n-1}\left\|\mathrm{D}_{[1]} \boldsymbol{v}\right\|_{\mathrm{L}^{2}(\mathcal{Q})}+\kappa_{2}^{n}\|\boldsymbol{v}\|_{\mathrm{L}^{2}(\mathcal{Q})} \\
& \left.+\kappa_{1}^{n-1}\left\|\partial_{X_{3}} \mathrm{D}_{[1]} v_{\alpha}\right\|_{\mathrm{L}^{2}(\mathcal{Q})}+\kappa_{1}^{n}\left\|\partial_{X_{3}} \boldsymbol{v}\right\|_{\mathrm{L}^{2}(\mathcal{Q})}\right) .
\end{aligned}
$$


Similarly, using (4.14b), we find

$$
-\mathrm{T}(\varepsilon) \mathrm{W}^{\text {asy }}(\varepsilon)=\varepsilon^{4} \sum_{0 \leq i \leq 4} \overline{\mathrm{T}}^{i}(\varepsilon) \mathrm{V}^{i}
$$

where $\overline{\mathrm{T}}^{i}(\varepsilon)$ are of order 1 in $\mathrm{D}_{\sigma}$ and 0 in $\varepsilon$ and depend of the operators $\mathrm{T}^{k}$ of (4.9). These series are given as convergent operators series, owing to estimates similar to (6.6).

Since $z$ is solution of $\left(P_{2 \mathrm{D}}\right)$, we have

$$
\mathrm{M} \boldsymbol{z}+\varepsilon^{2} \mathrm{~A}^{2} \boldsymbol{z}=\boldsymbol{g}+\varepsilon^{2}\left(\mathrm{~A}^{2}-\mathrm{B}\right) \boldsymbol{z} .
$$

Putting all together, we find

$$
\begin{aligned}
a_{3 \mathrm{D}}(\varepsilon)\left(\underline{\boldsymbol{u}}-\mathrm{U}^{\text {asy }}(\varepsilon) \boldsymbol{z}, \boldsymbol{v}\right) & =\varepsilon\langle\boldsymbol{f}-\boldsymbol{g}, \boldsymbol{v}\rangle_{\mathcal{Q} ; \varepsilon} \\
& +\varepsilon^{3}\left\langle\left(\mathrm{~A}^{2}-\mathrm{B}\right) \boldsymbol{z}, \boldsymbol{v}\right\rangle_{\mathcal{Q} ; \varepsilon} \\
& +\varepsilon^{4}\left\langle\mathrm{~L}^{1} \mathrm{~V}^{4} \boldsymbol{z}+\mathrm{L}^{2} \mathrm{~V}^{3} \boldsymbol{z}, \boldsymbol{v}\right\rangle_{\mathcal{Q} ; \varepsilon} \\
& +\varepsilon^{4}\left\langle\mathrm{~L}^{3} \mathrm{~V}^{2} \boldsymbol{z}+\mathrm{L}^{4} \mathrm{~V}^{1} \boldsymbol{z}+\mathrm{L}^{5} \mathrm{~V}^{0} \boldsymbol{z}, \boldsymbol{v}\right\rangle_{\mathcal{Q} ; \varepsilon} \\
& +\varepsilon^{5} \sum_{1 \leq i \leq 4}\left[\left\langle\mathrm{~L}^{i}(\varepsilon) \mathrm{V}^{i} \boldsymbol{z}, \boldsymbol{v}\right\rangle_{\mathcal{Q} ; \varepsilon}+\left\langle\overline{\mathrm{T}}^{i}(\varepsilon) \mathrm{V}^{i} \boldsymbol{z}, \boldsymbol{v}\right\rangle_{\Gamma_{ \pm} ; \varepsilon}\right]
\end{aligned}
$$

The proof of Lemma 6.2 consists in estimating each term in the above right hand side: Term (6.7a) in Sublemma 6.3.1, term (6.7b) in Sublemma 6.3.2, terms (6.7c) in Sublemma 6.3.3, and the remaining terms $(6.7 \mathrm{~d})$ and (6.7e) in the end of this proof of Lemma 6.2.

Sublemma 6.3.1 For $\boldsymbol{v} \in V(\mathcal{Q})$, we have the estimate:

$$
\varepsilon\langle\underline{\boldsymbol{f}}-\boldsymbol{g}, \boldsymbol{v}\rangle_{\mathcal{Q} ; \varepsilon} \lesssim \mathrm{D} E^{-1 / 2} \varepsilon\left\|\underline{\boldsymbol{f}}^{\mathrm{rem}}\right\|_{\mathrm{L}^{2}(\mathcal{Q})} \mathrm{E}_{3 \mathrm{D}}(\varepsilon)[\boldsymbol{v}]^{1 / 2}
$$

Proof of Sublemma 6.3.1. Let $G$ be the mean value operator

$$
\mathrm{G} \boldsymbol{v}=\frac{1}{2} \int_{-1}^{1} \boldsymbol{v}\left(X_{3}\right) \mathrm{d} X_{3}
$$

With (2.11), we obtain $\boldsymbol{g}=\mathrm{G} \underline{\boldsymbol{f}}$ and we compute

$$
\left|\varepsilon\langle\underline{\boldsymbol{f}}-\boldsymbol{g}, \boldsymbol{v}\rangle_{\mathcal{Q} ; \varepsilon}\right|=\left|\varepsilon\langle\underline{\boldsymbol{f}}-\mathrm{G} \underline{\boldsymbol{f}}, \boldsymbol{v}-\mathrm{G} \boldsymbol{v}\rangle_{\mathcal{Q} ; \varepsilon}\right| \leq \varepsilon\|\underline{\boldsymbol{f}}-\boldsymbol{g}\|_{\mathrm{L}^{2}(\mathcal{Q})}\|\boldsymbol{v}-\mathrm{G} \boldsymbol{v}\|_{\mathrm{L}^{2}(\mathcal{Q})} .
$$

Using the Bramble-Hilbert Lemma on $(-1,1)$, together with the fact that $X_{3}$ is an adimensional variable, we get

$$
\|\boldsymbol{v}-\mathrm{G} \boldsymbol{v}\|_{\mathrm{L}^{2}(\mathcal{Q})}^{2} \lesssim\left\|\partial_{X_{3}} \boldsymbol{v}\right\|_{\mathrm{L}^{2}(\mathcal{Q})}^{2} .
$$

Combining this with Korn inequality (4.6) we finally find

$$
\|\boldsymbol{v}-\mathrm{G} \boldsymbol{v}\|_{\mathrm{L}^{2}(\mathcal{Q})} \lesssim \mathrm{D} E^{-1 / 2} \varepsilon^{-1 / 2} \mathrm{E}_{3 \mathrm{D}}(\varepsilon)[\boldsymbol{v}]^{1 / 2}
$$

We conclude using $\underline{\boldsymbol{f}}^{\text {rem }}=\underline{\boldsymbol{f}}-\boldsymbol{g}$. 
Sublemma 6.3.2 For $\boldsymbol{v} \in V(\mathcal{Q})$, we have the estimate:

$$
\left|\varepsilon^{3}\left\langle\left(\mathrm{~A}^{2}-\mathrm{B}\right) \boldsymbol{z}, \boldsymbol{v}\right\rangle_{\mathcal{Q} ; \varepsilon}\right| \lesssim B_{S}^{1}(\varepsilon ; \boldsymbol{z})^{1 / 2} \mathrm{E}_{2 \mathrm{D}}^{\varepsilon}[\boldsymbol{z}]^{1 / 2} \mathrm{E}_{3 \mathrm{D}}(\varepsilon)[\boldsymbol{v}]^{1 / 2}
$$

where $B_{S}^{1}(\varepsilon ; \boldsymbol{z})$ is given by (6.2).

Proof of Sublemma 6.3.2. Using (4.15), we have for a 3D displacement $\boldsymbol{v}$ satisfying the homogeneous lateral boundary condition

$$
\begin{aligned}
\left|\left\langle\left(\mathrm{A}^{2}-\mathrm{B}\right) \boldsymbol{z}, \boldsymbol{v}\right\rangle_{\mathcal{Q} ; \varepsilon}\right| \lesssim E\left(|\boldsymbol{\gamma}(\boldsymbol{z})|_{2 ; S}+\kappa_{2}^{2}|\boldsymbol{z}|_{1 ; S}^{(\boldsymbol{b})}+\kappa_{1}\left|z_{3}\right|_{2 ; S}\right)\|\boldsymbol{\gamma}(\boldsymbol{v})\|_{\mathrm{L}^{2}(\mathcal{Q})} \\
+E\left(\kappa_{1}|\boldsymbol{\gamma}(\boldsymbol{z})|_{1 ; S}+\kappa_{2}^{2}|\boldsymbol{\gamma}(\boldsymbol{z})|_{0 ; S}\right)\left(\left\|\mathrm{D}_{\alpha} \boldsymbol{v}\right\|_{\mathrm{L}^{2}(\mathcal{Q})}+\kappa_{1}\|\boldsymbol{v}\|_{\mathrm{L}^{2}(\mathcal{Q})}\right) .
\end{aligned}
$$

But for any $v$ we have in non-shifted components (see (6.17c) and [19, Prop. 3.2])

$$
\gamma_{\alpha \beta}(\boldsymbol{v})=e_{\alpha \beta}(\varepsilon)(\boldsymbol{v})-\varepsilon X_{3}\left(c_{\alpha \beta} v_{3}+v_{\delta} \mathrm{D}_{\alpha} b_{\beta}^{\delta}\right) .
$$

Thus we have

$$
\|\gamma(\boldsymbol{v})\|_{\mathrm{L}^{2}(\mathcal{Q})} \lesssim\left\|e_{\alpha \beta}(\varepsilon)(\boldsymbol{v})\right\|_{\mathrm{L}^{2}(\mathcal{Q})}+\varepsilon \kappa_{2}^{2}\|\boldsymbol{v}\|_{\mathrm{L}^{2}(\mathcal{Q})} .
$$

Combining (6.11) with Korn inequalities (4.6) in (6.10) we find

$$
\begin{aligned}
\left|\left\langle\left(\mathrm{A}^{2}-\mathrm{B}\right) \boldsymbol{z}, \boldsymbol{v}\right\rangle_{\mathcal{Q} ; \varepsilon}\right| \lesssim \varepsilon^{-3 / 2} & E^{1 / 2} \mathrm{E}_{3 \mathrm{D}}(\varepsilon)[\boldsymbol{v}]^{1 / 2}\{ \\
& \varepsilon\left(|\boldsymbol{\gamma}(\boldsymbol{z})|_{2 ; S}+\kappa_{2}^{2}|\boldsymbol{z}|_{1 ; S}^{(\boldsymbol{b})}+\kappa_{1}\left|z_{3}\right|_{2 ; S}\right)\left(1+\mathrm{D}^{2} \kappa_{2}^{2}\right) \\
& \left.+\left(\kappa_{1}|\boldsymbol{\gamma}(\boldsymbol{z})|_{1 ; S}+\kappa_{2}^{2}|\gamma(\boldsymbol{z})|_{0 ; S}\right)\left(\mathrm{D}+\kappa_{1} \mathrm{D}^{2}\right)\right\} .
\end{aligned}
$$

Using (5.5) and the definition 2.4 of the wavelength $L_{n}$ we find using (4.5)

$$
\begin{aligned}
\left|\left\langle\left(\mathrm{A}^{2}-\mathrm{B}\right) \boldsymbol{z}, \boldsymbol{v}\right\rangle_{\mathcal{Q} ; \varepsilon}\right| \lesssim \varepsilon^{-3 / 2} & E^{1 / 2} \mathrm{E}_{3 \mathrm{D}}(\varepsilon)[\boldsymbol{v}]^{1 / 2}\{ \\
& \varepsilon\left(\left(L_{2}^{-2}+\kappa_{2}^{2}\right)|\boldsymbol{\gamma}(\boldsymbol{z})|_{0 ; S}+\kappa_{2}|\boldsymbol{\rho}(\boldsymbol{z})|_{0 ; S}\right)\left(1+\mathrm{D}^{2} \kappa_{2}^{2}\right) \\
& \left.+\left(\kappa_{1} L_{1}^{-1}+\kappa_{2}^{2}\right)|\boldsymbol{\gamma}(\boldsymbol{z})|_{0 ; S}\left(\mathrm{D}+\kappa_{1} \mathrm{D}^{2}\right)\right\}
\end{aligned}
$$

Using (5.8) we find

$$
\left|\varepsilon^{3}\left\langle\left(\mathrm{~A}^{2}-\mathrm{B}\right) \boldsymbol{z}, \boldsymbol{v}\right\rangle_{\mathcal{Q} ; \varepsilon}\right| \lesssim a_{S}(\varepsilon, \boldsymbol{z}) \mathrm{E}_{2 \mathrm{D}}^{\varepsilon}[\boldsymbol{z}]^{1 / 2} \mathrm{E}_{3 \mathrm{D}}(\varepsilon)[\boldsymbol{v}]^{1 / 2}
$$

where

$$
a_{S}(\varepsilon, \boldsymbol{z})=\varepsilon\left(\varepsilon L_{2}^{-2}+\varepsilon \kappa_{2}^{2}+\kappa_{2}\right)\left(1+\mathrm{D}^{2} \kappa_{2}^{2}\right)+\varepsilon\left(\kappa_{1} L_{1}^{-1}+\kappa_{2}^{2}\right)\left(\mathrm{D}+\kappa_{1} \mathrm{D}^{2}\right) .
$$

Using the definition of $\mathrm{r}=1 / \kappa_{5}$ and $L=L_{4}$ we can take

$$
a_{S}(\varepsilon, \boldsymbol{z})=\left(1+\frac{\mathrm{D}^{2}}{\mathrm{r}^{2}}\right)\left(\frac{\varepsilon}{L}+\frac{\varepsilon^{2}}{L^{2}}+\frac{\varepsilon}{\mathrm{r}}+\frac{\varepsilon^{2}}{\mathrm{r}^{2}}\right)
$$

and we get the result. 
Sublemma 6.3.3 For $v \in V(\mathcal{Q})$, we have the estimates

$$
\left|\varepsilon^{4}\left\langle\mathrm{~L}^{1} \mathrm{~V}^{4} \boldsymbol{z}, \boldsymbol{v}\right\rangle_{\mathcal{Q} ; \varepsilon}\right|+\left|\varepsilon^{4}\left\langle\mathrm{~L}^{2} \mathrm{~V}^{3} \boldsymbol{z}, \boldsymbol{v}\right\rangle_{\mathcal{Q} ; \varepsilon}\right| \lesssim B_{S}^{1}(\varepsilon ; \boldsymbol{z})^{1 / 2} \mathrm{E}_{2 \mathrm{D}}^{\varepsilon}[\boldsymbol{z}]^{1 / 2} \mathrm{E}_{3 \mathrm{D}}(\varepsilon)[\boldsymbol{v}]^{1 / 2},
$$

where $B_{S}^{1}(\varepsilon ; \boldsymbol{z})$ is given by (6.2).

Proof of Sublemma 6.3.3. The operators $\mathrm{V}^{3}$ and $\mathrm{V}^{4}$ are polynomials in $X_{3}$ with 2D operator coefficients. These operators are $\boldsymbol{b}$-homogeneous operators of degree 3 and 4 respectively. If $A$ is a $2 \mathrm{D}$ operator acting form $\Sigma(S)$ into itself, it can be viewed as a block $2 \times 2$ matrix

$$
A=\left(\begin{array}{cc}
A_{\sigma \alpha} & A_{\sigma 3} \\
A_{3 \alpha} & A_{33}
\end{array}\right)
$$

and the inequality

$$
\operatorname{deg} A \leq\left(\begin{array}{ll}
a_{* *} & a_{* 3} \\
a_{3 *} & a_{33}
\end{array}\right)
$$

means that $\operatorname{deg} A_{\sigma \alpha} \leq a_{* *}$, etc..., where deg means the order as partial differential operator. With these notations, we have (see [19, Prop. 4.2]) that

$$
\operatorname{deg} \mathrm{V}^{3} \leq\left(\begin{array}{ll}
2 & 3 \\
3 & 2
\end{array}\right) \quad \text { and } \quad \operatorname{deg} \mathrm{V}^{4} \leq\left(\begin{array}{ll}
4 & 3 \\
3 & 4
\end{array}\right)
$$

(i) Using the expression of the operator $\mathrm{L}^{1}$ (see [19, Thm. 3.3]),

$$
\begin{aligned}
& \mathrm{L}_{\sigma}^{1}(\boldsymbol{w})=-\mu b_{\alpha}^{\alpha} \partial_{X_{3}} w_{\sigma}+(\lambda+\mu) \mathrm{D}_{\sigma} \partial_{X_{3}} w_{3}-X_{3} \mu b_{\sigma}^{\alpha} \partial_{X_{3}}^{2} w_{\alpha}, \\
& \mathrm{L}_{3}^{1}(\boldsymbol{w})=-\mu b_{\alpha}^{\alpha} \partial_{X_{3}} w_{3}+(\lambda+\mu) \gamma_{\alpha}^{\alpha}\left(\partial_{X_{3}} \boldsymbol{w}\right),
\end{aligned}
$$

and the identity $\gamma_{\alpha}^{\alpha}(\boldsymbol{u})=\mathrm{D}^{\alpha} u_{\alpha}-b_{\alpha}^{\alpha} u_{3}$, we find

$$
\begin{array}{r}
\left\langle\mathrm{L}^{1} \mathrm{~V}^{4} \boldsymbol{z}, \boldsymbol{v}\right\rangle_{\mathcal{Q} ; \varepsilon}=\int_{\mathcal{Q}}\left(-\mu b_{\alpha}^{\alpha} \partial_{X_{3}} \mathrm{~V}_{\sigma}^{4} \boldsymbol{z}+(\lambda+\mu) \mathrm{D}_{\sigma} \partial_{X_{3}} \mathrm{~V}_{3}^{4} \boldsymbol{z}-X_{3} \mu b_{\sigma}^{\alpha} \partial_{X_{3}}^{2} \mathrm{~V}_{\alpha}^{4} \boldsymbol{z}\right) v^{\sigma} \mathrm{d} V \\
+\int_{\mathcal{Q}}\left(-(\lambda+2 \mu) b_{\alpha}^{\alpha} \partial_{X_{3}} \mathrm{~V}_{3}^{4} \boldsymbol{z}+(\lambda+\mu) \mathrm{D}^{\alpha} \partial_{X_{3}} \mathrm{~V}_{\alpha}^{4} \boldsymbol{z}\right) v_{3} \mathrm{~d} V
\end{array}
$$

where $\mathrm{d} V=\left(1+h\left(\varepsilon X_{3}\right)\right) \mathrm{d} S \mathrm{~d} X_{3}$ (cf. (4.8)). Using the fact that $\left.\boldsymbol{v}\right|_{\Gamma_{0}}=0$ we can integrate by parts with respect to the surfacic derivative $\mathrm{D}_{\sigma}$, and we obtain (we omit $\mathrm{d} V$ ):

$$
\begin{array}{r}
\left\langle\mathrm{L}^{1} \mathrm{~V}^{4} \boldsymbol{z}, \boldsymbol{v}\right\rangle_{\mathcal{Q} ; \varepsilon}=-\int_{\mathcal{Q}}\left(\mu b_{\alpha}^{\alpha} \partial_{X_{3}} \mathrm{~V}_{\sigma}^{4} \boldsymbol{z}+X_{3} \mu b_{\sigma}^{\alpha} \partial_{X_{3}}^{2} \mathrm{~V}_{\alpha}^{4} \boldsymbol{z}\right) v^{\sigma}-\int_{\mathcal{Q}}(\lambda+\mu)\left(\partial_{X_{3}} \mathrm{~V}_{3}^{4} \boldsymbol{z}\right) \mathrm{D}_{\sigma} v^{\sigma} \\
-\int_{\mathcal{Q}}(\lambda+2 \mu)\left(\partial_{X_{3}} \mathrm{~V}_{3}^{4} \boldsymbol{z}\right) b_{\alpha}^{\alpha} v_{3}-\int_{\mathcal{Q}}(\lambda+\mu)\left(\partial_{X_{3}} \mathrm{~V}_{\alpha}^{4} \boldsymbol{z}\right) \mathrm{D}^{\alpha} v_{3}
\end{array}
$$

and hence

$$
\begin{array}{r}
\left\langle\mathrm{L}^{1} \mathrm{~V}^{4} \boldsymbol{z}, \boldsymbol{v}\right\rangle_{\mathcal{Q} ; \varepsilon}=-\int_{\mathcal{Q}}\left(\mu b_{\alpha}^{\alpha} \partial_{X_{3}} \mathrm{~V}_{\sigma}^{4} \boldsymbol{z}+X_{3} \mu b_{\sigma}^{\alpha} \partial_{X_{3}}^{2} \mathrm{~V}_{\alpha}^{4} \boldsymbol{z}\right) v^{\sigma}-\int_{\mathcal{Q}} \mu\left(\partial_{X_{3}} \mathrm{~V}_{3}^{4} \boldsymbol{z}\right) b_{\alpha}^{\alpha} v_{3} \\
-\int_{\mathcal{Q}}(\lambda+\mu)\left(\partial_{X_{3}} \mathrm{~V}_{3}^{4} \boldsymbol{z}\right) \gamma_{\sigma}^{\sigma}(\boldsymbol{v})-\int_{\mathcal{Q}}(\lambda+\mu)\left(\partial_{X_{3}} \mathrm{~V}_{\alpha}^{4} \boldsymbol{z}\right) \mathrm{D}^{\alpha} v_{3}
\end{array}
$$


The operator $V_{3}^{4}$ is $\boldsymbol{b}$-homogeneous of degree 4 , and of orders of derivative 3 in $z_{\sigma}$ and 4 in $z_{3}$. By integration by parts using the boundary condition on $\boldsymbol{v}$, we obtain

$$
\begin{array}{r}
\left|\int_{\mathcal{Q}} \mu\left(\partial_{X_{3}} \mathrm{~V}_{3}^{4} \boldsymbol{z}\right) b_{\alpha}^{\alpha} v_{3}\right| \lesssim E\left(\sum_{j=0}^{3} \kappa_{4}^{4-j}|\boldsymbol{z}|_{j ; S}\right) \kappa_{1}\left\|v_{3}\right\|_{\mathrm{L}^{2}(\mathcal{Q})}+E \kappa_{1}\left|z_{3}\right|_{3 ; S}\left\|\mathrm{D}_{\alpha} v_{3}\right\|_{\mathrm{L}^{2}(\mathcal{Q})} \\
\lesssim E\left(\sum_{j=0}^{3} \kappa_{4}^{4-j}|\boldsymbol{z}|_{j ; S}\right)\left(\left\|\mathrm{D}_{\alpha} v_{3}\right\|_{\mathrm{L}^{2}(\mathcal{Q})}+\kappa_{4}\left\|v_{3}\right\|_{\mathrm{L}^{2}(\mathcal{Q})}\right) .
\end{array}
$$

As the operator $V_{\alpha}^{4}$ is $\boldsymbol{b}$-homogeneous of degree 4 , and of orders of derivative 3 in $z_{3}$ and 4 in $z_{\alpha}$, we obtain

$$
\begin{aligned}
\left|\left\langle\mathrm{L}^{1} \mathrm{~V}^{4} \boldsymbol{z}, \boldsymbol{v}\right\rangle_{\mathcal{Q} ; \varepsilon}\right| \lesssim E\left(\left|z_{\alpha}\right|_{4 ; S}+\right. & \left.\sum_{j=0}^{3} \kappa_{4}^{4-j}|\boldsymbol{z}|_{j ; S}\right)\left(\left\|\mathrm{D}_{\alpha} \boldsymbol{v}\right\|_{\mathrm{L}^{2}(\mathcal{Q})}+\kappa_{4}\|\boldsymbol{v}\|_{\mathrm{L}^{2}(\mathcal{Q})}\right) \\
& +E\left(\left|z_{3}\right|_{4 ; S}+\sum_{j=0}^{3} \kappa_{4}^{4-j}|\boldsymbol{z}|_{j ; S}\right)\left\|\gamma_{\alpha \beta}(\boldsymbol{v})\right\|_{\mathrm{L}^{2}(\mathcal{Q})}
\end{aligned}
$$

Using (6.11), the Korn inequalities (4.6) and inequality (5.8) we find

$$
\begin{array}{r}
\left|\left\langle\mathrm{L}^{1} \mathrm{~V}^{4} \boldsymbol{z}, \boldsymbol{v}\right\rangle_{\mathcal{Q} ; \varepsilon}\right| \lesssim \varepsilon^{-3 / 2} E^{1 / 2}\left(\left|z_{\alpha}\right|_{4 ; S}+\sum_{j=0}^{3} \kappa_{4}^{4-j}|\boldsymbol{z}|_{j ; S}\right)\left(\mathrm{D}+\mathrm{D}^{2} \kappa_{4}\right) \mathrm{E}_{3 \mathrm{D}}(\varepsilon)[\boldsymbol{v}]^{1 / 2} \\
+\varepsilon^{-1 / 2} E^{1 / 2}\left(\left|z_{3}\right|_{4 ; S}+\sum_{j=0}^{3} \kappa_{4}^{4-j}|\boldsymbol{z}|_{j ; S}\right) \mathrm{E}_{3 \mathrm{D}}(\varepsilon)[\boldsymbol{v}]^{1 / 2}
\end{array}
$$

Recall that $\kappa=\kappa_{5}$ and $L=L_{4}$. Here, because of asymmetry between surfacic and transverse components, we do not use estimates (5.9): We obtain sharper estimates using directly (5.6a) and (5.6b),

$$
\begin{aligned}
\left|z_{\alpha}\right|_{4 ; S}+\sum_{j=0}^{3} \kappa_{4}^{4-j}|\boldsymbol{z}|_{j ; S} \lesssim \sum_{j=0}^{3} & \kappa^{3-j}|\boldsymbol{\gamma}(\boldsymbol{z})|_{j ; S}+\sum_{j=0}^{1} \kappa^{2-j}|\boldsymbol{\rho}(\boldsymbol{z})|_{j ; S} \\
& \lesssim \varepsilon^{-3 / 2} E^{-1 / 2}\left(\varepsilon \sum_{j=0}^{3} \kappa^{3-j} L^{-j}+\sum_{j=0}^{1} \kappa^{2-j} L^{-j}\right) \mathrm{E}_{2 \mathrm{D}}^{\varepsilon}[\boldsymbol{z}]^{1 / 2}
\end{aligned}
$$

Similarly, we have

$$
\begin{aligned}
\left|z_{3}\right|_{4 ; S}+\sum_{j=0}^{3} \kappa_{4}^{4-j}|\boldsymbol{z}|_{j ; S} \lesssim \sum_{j=0}^{2} & \kappa^{2-j}|\boldsymbol{\rho}(\boldsymbol{z})|_{j ; S}+\sum_{j=0}^{2} \kappa^{3-j}|\boldsymbol{\gamma}(\boldsymbol{z})|_{j ; S} \\
& \lesssim \varepsilon^{-3 / 2} E^{-1 / 2}\left(\sum_{j=0}^{2} \kappa^{2-j} L^{-j}+\varepsilon \sum_{j=0}^{2} \kappa^{3-j} L^{-j}\right) \mathrm{E}_{2 \mathrm{D}}^{\varepsilon}[\boldsymbol{z}]^{1 / 2} .
\end{aligned}
$$

Combining these estimates with (6.14) we find

$$
\left|\left\langle\varepsilon^{4} \mathrm{~L}^{1} \mathrm{~V}^{4} \boldsymbol{z}, \boldsymbol{v}\right\rangle_{\mathcal{Q} ; \varepsilon}\right| \lesssim b_{S}(\boldsymbol{z}, \varepsilon) \mathrm{E}_{2 \mathrm{D}}^{\varepsilon}[\boldsymbol{z}]^{1 / 2} \mathrm{E}_{3 \mathrm{D}}(\varepsilon)[\boldsymbol{v}]^{1 / 2}
$$


with

$$
\begin{aligned}
b_{S}(\boldsymbol{z}, \varepsilon)=\varepsilon\left(\mathrm{D}+\mathrm{D}^{2} \kappa\right)\left(\varepsilon L^{-3}+\varepsilon \sum_{j=0}^{2} \kappa^{3-j} L^{-j}\right. & \left.+\sum_{j=0}^{1} \kappa^{2-j} L^{-j}\right) \\
& +\varepsilon^{2}\left(\sum_{j=0}^{2} \kappa^{2-j} L^{-j}+\varepsilon \sum_{j=0}^{2} \kappa^{3-j} L^{-j}\right)
\end{aligned}
$$

and we check that

$$
b_{S}(\boldsymbol{z}, \varepsilon) \lesssim \frac{\varepsilon^{2}}{L^{3}}\left(\mathrm{D}+\frac{\mathrm{D}^{2}}{\mathrm{r}}\right)+\left(1+\frac{\mathrm{D}^{2}}{\mathrm{r}^{2}}\right)\left(\sum_{\substack{i+j=k \\ k \in\{1,2\}}} \frac{\varepsilon^{k}}{\mathrm{r}^{i} L^{j}}\right)
$$

and hence $b_{S}(\boldsymbol{z}, \varepsilon) \lesssim B_{S}^{1}(\boldsymbol{z} ; \varepsilon)^{1 / 2}$. Note that we only need the introduction of $\kappa_{4}$ and $L_{3}$ to obtain this estimate.

(ii) Similarly, using the degrees (6.12) of $\mathrm{V}^{3}$ and the expression of $\mathrm{L}^{2}$ cf. [19, Prop. 3.3]

$$
\begin{array}{r}
\mathrm{L}_{\sigma}^{2}(\boldsymbol{w})=-\mu X_{3} c_{\alpha}^{\alpha} \partial_{X_{3}} w_{\sigma}+\mu X_{3} b_{\alpha}^{\alpha} b_{\sigma}^{\beta} \partial_{X_{3}} w_{\beta}-\mu b_{\alpha}^{\alpha} \mathrm{D}_{\sigma} w_{3}-\mu b_{\beta}^{\beta} b_{\sigma}^{\alpha} w_{\alpha} \\
+\lambda \mathrm{D}_{\sigma} \gamma_{\alpha}^{\alpha}(\boldsymbol{w}) \\
+2 \mu \mathrm{D}_{\alpha} \gamma_{\sigma}^{\alpha}(\boldsymbol{w}), \\
\mathrm{L}_{3}^{2}(\boldsymbol{w})=-\mu X_{3} c_{\alpha}^{\alpha} \partial_{X_{3}} w_{3}+(\lambda+\mu) b_{\alpha}^{\beta} \gamma_{\beta}^{\alpha}\left(\partial_{X_{3}}\left(X_{3} \boldsymbol{w}\right)\right)+\mu b_{\alpha}^{\beta} \gamma_{\beta}^{\alpha}(\boldsymbol{w})+\mu \mathrm{D}^{\alpha} \theta_{\alpha}(\boldsymbol{w}),
\end{array}
$$

we have after integration by parts that

$$
\begin{aligned}
\left\langle\mathrm{L}^{2} \mathrm{~V}^{3} \boldsymbol{z}, \boldsymbol{v}\right\rangle_{\mathcal{Q} ; \varepsilon}=-\int_{\mathcal{Q}}\left(\mu X_{3} c_{\alpha}^{\alpha} \partial_{X_{3}} \mathrm{~V}_{\sigma}^{3} \boldsymbol{z}-\mu X_{3} b_{\alpha}^{\alpha} b_{\sigma}^{\beta} \partial_{X_{3}} \mathrm{~V}_{\beta}^{3} \boldsymbol{z}+\mu b_{\beta}^{\beta} b_{\sigma}^{\alpha} \mathrm{V}_{\alpha}^{3} \boldsymbol{z}\right) v^{\sigma} \mathrm{d} V \\
+\int_{\mathcal{Q}}\left(\mu b_{\alpha}^{\alpha} \mathrm{V}_{3}^{3} \boldsymbol{z}-\lambda \gamma_{\alpha}^{\alpha}\left(\mathrm{V}^{3} \boldsymbol{z}\right)\right) \mathrm{D}_{\sigma} v^{\sigma} \mathrm{d} V-\int_{\mathcal{Q}} 2 \mu \gamma_{\sigma}^{\alpha}\left(\mathrm{V}^{3} \boldsymbol{z}\right) \mathrm{D}_{\alpha} v^{\sigma} \mathrm{d} V \\
-\int_{\mathcal{Q}}(\lambda+2 \mu)\left(c_{\alpha}^{\alpha} \partial_{3}\left(X_{3} \mathrm{~V}_{3}^{3} \boldsymbol{z}\right) v_{3} \mathrm{~d} V-\int_{\mathcal{Q}}\left((\lambda+\mu) \partial_{X_{3}}\left(X_{3} \mathrm{~V}_{\alpha}^{3} \boldsymbol{z}\right)\right)\left(\mathrm{D}^{\beta} b_{\beta}^{\alpha} v_{3}\right) \mathrm{d} V\right. \\
-\int_{\mathcal{Q}}\left(\mu \mathrm{V}_{\alpha}^{3} \boldsymbol{z}\right)\left(\mathrm{D}^{\beta} b_{\beta}^{\alpha} v_{3}\right) \mathrm{d} V-\int_{\mathcal{Q}} \mu \theta_{\alpha}\left(\mathrm{V}^{3} \boldsymbol{z}\right)\left(\mathrm{D}^{\alpha} v_{3}\right) \mathrm{d} V .
\end{aligned}
$$

Using the relation

$$
\tau^{\alpha \beta} \mathrm{D}_{\alpha} w_{\beta}=\tau_{\alpha}^{\beta} \gamma_{\beta}^{\alpha}(\boldsymbol{w})+\tau_{\alpha}^{\beta} b_{\beta}^{\alpha} w_{3}
$$

valid for any symmetric tensor $\tau_{\alpha \beta}$, and using integration by parts, we find the same estimate as in (6.13) which yields the result.

End of proof of Lemma 6.2. We now prove that the remaining terms (6.7d) and (6.7e) in equation (6.7) can be estimated by terms of the form

$$
B_{S}^{1}(\varepsilon ; \boldsymbol{z})^{1 / 2} \mathrm{E}_{3 \mathrm{D}}^{\varepsilon}[\boldsymbol{z}]^{1 / 2} \mathrm{E}_{3 \mathrm{D}}(\varepsilon)[\boldsymbol{v}]
$$

where the expression of the bound $B_{S}^{1}$ is given by (6.2). 
Using [19, Thm. 3.3], we can prove like for the estimates (6.6) the following uniform bound for all $n \geq 3, \boldsymbol{w} \in \mathrm{H}^{1}(\mathcal{Q})^{3}$ and $\boldsymbol{v} \in V(\mathcal{Q})$ :

$$
\begin{aligned}
\left|\left\langle\mathrm{L}^{n} \boldsymbol{w}, \boldsymbol{v}\right\rangle_{\mathcal{Q} ; \varepsilon}\right| \lesssim & n E \kappa_{2}^{n-2}\left(\left\|\mathrm{D}_{\alpha} \boldsymbol{v}\right\|_{\mathrm{L}^{2}(\mathcal{Q})}+\kappa_{2}\|\boldsymbol{v}\|_{\mathrm{L}^{2}(\mathcal{Q})}\right) \times \\
& \left(\left\|\mathrm{D}_{\alpha} \boldsymbol{w}\right\|_{\mathrm{L}^{2}(\mathcal{Q})}+\kappa_{2}\|\boldsymbol{w}\|_{\mathrm{L}^{2}(\mathcal{Q})}+\left\|\partial_{X_{3}} \mathrm{D}_{\alpha} \boldsymbol{w}\right\|_{\mathrm{L}^{2}(\mathcal{Q})}+\kappa_{2}\left\|\partial_{X_{3}} \boldsymbol{w}\right\|_{\mathrm{L}^{2}(\mathcal{Q})}\right) .
\end{aligned}
$$

Recall that for all $n \geq 0$ the operators $\mathrm{V}^{n}$ are $\boldsymbol{b}$-homogeneous of degree $n$. Hence the uniform estimates for all $n \geq 3$ and all $i \in\{0,1,2,3,4\}$

$$
\begin{aligned}
\left|\left\langle\mathrm{L}^{n} \mathrm{~V}^{i} \boldsymbol{z}, \boldsymbol{v}\right\rangle_{\mathcal{Q} ; \varepsilon)}\right| & \\
& \lesssim n E \kappa_{2}^{n-2}\left(\left\|\mathrm{D}_{\alpha} \boldsymbol{v}\right\|_{\mathrm{L}^{2}(\mathcal{Q})}+\kappa_{2}\|\boldsymbol{v}\|_{\mathrm{L}^{2}(\mathcal{Q})}\right)\left(|\boldsymbol{z}|_{i+1 ; S}^{(\boldsymbol{b})}+\kappa_{2}|\boldsymbol{z}|_{i ; S}^{(\boldsymbol{b})}\right) \\
& \lesssim n E^{1 / 2} \varepsilon^{-3 / 2} \kappa_{2}^{n-2}\left(\mathrm{D}+\mathrm{D}^{2} \kappa_{2}\right) \mathrm{E}_{3 \mathrm{D}}(\varepsilon)[\boldsymbol{v}]^{1 / 2} \cdot\left(|\boldsymbol{z}|_{i+1 ; S}^{(\boldsymbol{b})}+\kappa_{2}|\boldsymbol{z}|_{i ; S}^{(\boldsymbol{b})}\right),
\end{aligned}
$$

using Korn inequalities (4.6).

This estimate yields immediately that

$$
\begin{aligned}
\left|\varepsilon^{4}\left\langle\mathrm{~L}^{3} \mathrm{~V}^{2} \boldsymbol{z}+\mathrm{L}^{4} \mathrm{~V}^{1} \boldsymbol{z}+\mathrm{L}^{5} \mathrm{~V}^{0} \boldsymbol{z}, \boldsymbol{v}\right\rangle_{\mathcal{Q} ; \varepsilon}\right| & \\
& \lesssim \varepsilon^{5 / 2} E^{1 / 2} \kappa_{2}\left(\mathrm{D}+\mathrm{D}^{2} \kappa_{2}\right)\left(\sum_{i+j=3} \kappa_{2}^{i}|\boldsymbol{z}|_{j ; S}^{(\boldsymbol{b})}\right) \mathrm{E}_{3 \mathrm{D}}(\varepsilon)[\boldsymbol{v}]^{1 / 2},
\end{aligned}
$$

and using the a priori estimate (5.9) we get

$$
\begin{aligned}
\left|\varepsilon^{4}\left\langle\mathrm{~L}^{3} \mathrm{~V}^{2} \boldsymbol{z}+\mathrm{L}^{4} \mathrm{~V}^{1} \boldsymbol{z}+\mathrm{L}^{5} \mathrm{~V}^{0} \boldsymbol{z}, \boldsymbol{v}\right\rangle_{\mathcal{Q} ; \varepsilon}\right| & \\
& \lesssim \varepsilon\left(\mathrm{D} \kappa+\mathrm{D}^{2} \kappa^{2}\right)\left(\sum_{i, j, k \in G_{3}} \varepsilon^{k} \kappa^{i} L^{-j}\right) \mathrm{E}_{2 \mathrm{D}}^{\varepsilon}[\boldsymbol{z}]^{1 / 2} \mathrm{E}_{3 \mathrm{D}}(\varepsilon)[\boldsymbol{v}]^{1 / 2},
\end{aligned}
$$

where $G_{3}$ is given by the formula (5.10).

But the constant in the right-hand side can be written

$$
\left(\frac{\mathrm{D}}{\mathrm{r}}+\frac{\mathrm{D}^{2}}{\mathrm{r}^{2}}\right)\left(\sum_{\substack{i+j=k+1 \\ k \in\{0,1\}}} \frac{\varepsilon^{k+1}}{\mathrm{r}^{i} L^{j}}\right) \lesssim B_{S}^{1}(\varepsilon ; \boldsymbol{z})^{1 / 2}
$$

after a change of index $k \mapsto k-1$ in the sum. This yields the result. Note we only need $\kappa_{3}$ and $L_{2}$ to obtain this bound .

The operators $\bar{L}^{i}$ in the term (6.7e) are the power series (6.5) of the operators $\mathrm{L}^{k}$. Using (6.15) and the fact that $\varepsilon \leq r$, we derive that

$$
\left|\varepsilon^{5}\left\langle\sum_{i=0}^{4} \overline{\mathrm{L}}^{i}(\varepsilon) \mathrm{V}^{i} \boldsymbol{z}, \boldsymbol{v}\right\rangle_{\mathcal{Q} ; \varepsilon}\right| \lesssim \varepsilon^{7 / 2} E^{1 / 2} \mathrm{D}\left(1+\mathrm{D} \kappa_{2}\right) \mathrm{E}_{3 \mathrm{D}}(\varepsilon)[\boldsymbol{v}]^{1 / 2} \sum_{i=0}^{4}\left(|\boldsymbol{z}|_{i+1 ; S}^{(\boldsymbol{b})}+\kappa_{2}|\boldsymbol{z}|_{i ; S}^{(\boldsymbol{b})}\right)
$$


Owing to (5.9) for $n=5$, this shows that

$$
\left|\varepsilon^{5}\left\langle\sum_{i=0}^{4} \overline{\mathrm{L}}^{i}(\varepsilon) \mathrm{V}^{i} \boldsymbol{z}, \boldsymbol{v}\right\rangle_{\mathcal{Q} ; \varepsilon}\right| \lesssim \varepsilon^{2} \mathrm{D}\left(1+\mathrm{D} \kappa_{3}\right)\left(\sum_{i, j, k \in G_{5}} \varepsilon^{k} \kappa^{i} L^{-j}\right) \mathrm{E}_{2 \mathrm{D}}^{\varepsilon}[\boldsymbol{z}]^{1 / 2} \mathrm{E}_{3 \mathrm{D}}(\varepsilon)[\boldsymbol{v}]^{1 / 2} .
$$

But the constant in the right hand side is smaller than

$$
\left(1+\frac{\mathrm{D}}{\mathrm{r}}\right) \sum_{\substack{i+j=k+1 \\ k \in\{2,3\}}} \frac{\varepsilon^{k} \mathrm{D}}{\mathrm{r}^{i} L^{j}} \lesssim\left(1+\frac{\mathrm{D}}{\mathrm{r}}\right)\left(\frac{\varepsilon^{2} \mathrm{D}}{L^{3}}+\frac{\varepsilon^{3} \mathrm{D}}{L^{4}}\right)+\left(\frac{\mathrm{D}}{\mathrm{r}}+\frac{\mathrm{D}^{2}}{\mathrm{r}^{2}}\right) \sum_{\substack{i+j=k \\ k \in\{2,3\}}} \frac{\varepsilon^{k}}{\mathrm{r}^{i} L^{j}}
$$

after separating in the sum the terms where $i=0$ and those for $i \geq 1$. This term is dominated by $B_{S}^{1}(\varepsilon ; \boldsymbol{z})^{1 / 2}$. Note that we only need $\kappa_{5}$ and $L_{4}$ to obtain this bound. The estimate for the traction terms involving the operators $\overline{\mathrm{T}}^{i}(\varepsilon)$ can be done similarly, and this proves the lemma.

We can now prove the main result of this section.

Proof of Proposition 6.1. Using Lemma 6.2 we have

$$
\begin{aligned}
a_{3 \mathrm{D}}(\varepsilon)(\underline{\boldsymbol{u}}-\mathrm{U}(\varepsilon) \boldsymbol{z}, \boldsymbol{v}) & =a_{3 \mathrm{D}}(\varepsilon)\left(\underline{\boldsymbol{u}}-\mathrm{U}^{\text {asy }}(\varepsilon \boldsymbol{z}, \boldsymbol{v})+a_{3 \mathrm{D}}(\varepsilon)\left(\mathrm{U}(\varepsilon) \boldsymbol{z}-\mathrm{U}^{\text {asy }}(\varepsilon) \boldsymbol{z}, \boldsymbol{v}\right)\right. \\
& \lesssim\left(B_{1}^{1 / 2}+\mathrm{E}_{3 \mathrm{D}}(\varepsilon)\left[\mathrm{U}(\varepsilon) \boldsymbol{z}-\mathrm{U}^{\text {asy }}(\varepsilon) \boldsymbol{z}\right]^{1 / 2}\right) \mathrm{E}_{3 \mathrm{D}}(\varepsilon)[\boldsymbol{v}]^{1 / 2} .
\end{aligned}
$$

Thus the proposition is proved provided we show $\mathrm{E}_{3 \mathrm{D}}(\varepsilon)\left[\mathrm{U}(\varepsilon) \boldsymbol{z}-\mathrm{U}^{\text {asy }}(\varepsilon) \boldsymbol{z}\right] \lesssim B_{1}$, or equivalently $\widetilde{\mathrm{E}}_{3 \mathrm{D}}(\varepsilon)\left[\mathrm{W}(\varepsilon) \boldsymbol{z}-\mathrm{W}^{\text {asy }}(\varepsilon) \boldsymbol{z}\right] \lesssim B_{1}$. Here the scaled shifted energy $\widetilde{\mathrm{E}}_{3 \mathrm{D}}(\varepsilon)[\boldsymbol{v}]$ is given by, for $\boldsymbol{v} \in V(\mathcal{Q})$

$$
\begin{aligned}
& \widetilde{\mathrm{E}}_{3 \mathrm{D}}(\varepsilon)[\boldsymbol{v}]=\int_{\mathcal{Q}} A^{i j k \ell}(\varepsilon) \tilde{e}_{i j}(\varepsilon)(\boldsymbol{v}) \tilde{e}_{k \ell}(\varepsilon)(\boldsymbol{v}) \mathrm{d} V \\
& =\int_{\mathcal{Q}}\left[(\lambda+2 \mu) \tilde{e}_{3}^{3}(\varepsilon)(\boldsymbol{w}) \tilde{e}_{3}^{3}(\varepsilon)(\boldsymbol{w})+2 \lambda \tilde{e}_{3}^{3}(\varepsilon)(\boldsymbol{w}) \tilde{e}_{\alpha}^{\alpha}(\varepsilon)(\boldsymbol{w})+\lambda \tilde{e}_{\alpha}^{\alpha}(\varepsilon)(\boldsymbol{w}) \tilde{e}_{\beta}^{\beta}(\varepsilon)(\boldsymbol{w})\right. \\
& \left.\quad+4 \mu a^{\alpha \beta}(\varepsilon)\left(x_{3}\right) \tilde{e}_{\alpha}^{3}(\varepsilon)(\boldsymbol{w}) \tilde{e}_{\beta}^{3}(\varepsilon)(\boldsymbol{w})+2 \mu \tilde{e}_{\alpha}^{\beta}(\varepsilon)(\boldsymbol{w}) \tilde{e}_{\beta}^{\alpha}(\varepsilon)(\boldsymbol{w})\right] \mathrm{d} V, \quad(6
\end{aligned}
$$

where $a^{\alpha \beta}(\varepsilon)\left(x_{3}\right)=\left(\mu^{-1}\left(\varepsilon X_{3}\right)\right)_{\sigma}^{\alpha} a^{\sigma \nu}\left(\mu^{-1}\left(\varepsilon X_{3}\right)\right)_{\nu}^{\beta}$ is the inverse of the metric tensor of the surface at the level $x_{3}=\varepsilon X_{3}$ in the shell, see $[32,19]$. The inverse $\left(\mu^{-1}\left(\varepsilon X_{3}\right)\right)_{\sigma}^{\alpha}$ of the shifter can be expanded as

$$
\left(\mu^{-1}\left(\varepsilon X_{3}\right)\right)_{\sigma}^{\alpha}=\sum_{k=0}^{\infty} \varepsilon^{k} X_{3}^{k}\left(b^{k}\right)_{\sigma}^{\alpha}
$$

The shifted tensor $\tilde{e}_{i}^{j}(\varepsilon)(\boldsymbol{w})$ has the expression in normal coordinates, cf. [19]:

$$
\begin{aligned}
& \tilde{e}_{3}^{3}(\varepsilon)(\boldsymbol{w})=\varepsilon^{-1} \partial_{X_{3}} w_{3} \\
& \tilde{e}_{\beta}^{3}(\varepsilon)(\boldsymbol{w})=\frac{1}{2}\left(\varepsilon^{-1} \partial_{X_{3}} w_{\beta}-X_{3} b_{\beta}^{\alpha} \partial_{x_{3}} w_{\alpha}+\theta_{\beta}(\boldsymbol{w})\right) \\
& \tilde{e}_{\beta}^{\alpha}(\varepsilon)(\boldsymbol{w})=\gamma_{\beta}^{\alpha}(\boldsymbol{w})+\sum_{n=1}^{\infty} \varepsilon^{n} X_{3}^{n}\left(b^{n}\right)_{\delta}^{\alpha} \gamma_{\beta}^{\delta}(\boldsymbol{w})+\sum_{n=1}^{\infty} n \varepsilon^{n} X_{3}^{n}\left(b^{n-1}\right)_{\delta}^{\alpha} \Lambda_{\cdot \beta}^{\delta} \cdot(\boldsymbol{w}),
\end{aligned}
$$


where $\theta_{\beta}(\boldsymbol{z})=\mathrm{D}_{\beta} z_{3}+b_{\beta}^{\alpha} z_{\alpha}$ and $\Lambda_{\alpha \beta}(\boldsymbol{z})=\frac{1}{2}\left(b_{\alpha}^{\sigma} \mathrm{D}_{\sigma} z_{\beta}-b_{\beta}^{\sigma} \mathrm{D}_{\alpha} z_{\sigma}\right)$.

By definition, we have

$$
\mathrm{W}^{\text {asy }}(\varepsilon) \boldsymbol{z}=\mathrm{W}(\varepsilon) \boldsymbol{z}+\varepsilon^{2} \mathrm{v}^{2} \boldsymbol{z}+\varepsilon^{3} \mathrm{~V}^{3} \boldsymbol{z}+\varepsilon^{4} \mathrm{~V}^{4} \boldsymbol{z}
$$

where

$$
\mathrm{v}^{2} \boldsymbol{z}=\left\{\begin{array}{l}
\frac{X_{3}^{2}}{2} p \mathrm{D}_{\sigma} \gamma_{\alpha}^{\alpha}(\boldsymbol{z}) \\
\frac{X_{3}^{2}}{2}\left(-p^{2} b_{\alpha}^{\alpha} \gamma_{\beta}^{\beta}(\boldsymbol{z})-2 p b_{\alpha}^{\beta} \gamma_{\beta}^{\alpha}(\boldsymbol{z})\right)
\end{array}\right.
$$

We now successively estimate the energy of the three terms $\mathrm{v}^{2} \boldsymbol{z}, \mathrm{V}^{3} \boldsymbol{z}$ and $\mathrm{V}^{4} \boldsymbol{z}$.

Using (6.16) we have for all $\boldsymbol{v} \in \mathrm{H}^{1}(\mathcal{Q})$

$$
\widetilde{\mathrm{E}}_{3 \mathrm{D}}(\varepsilon)[\boldsymbol{v}] \lesssim \varepsilon E\left(\left\|\tilde{e}_{\beta}^{\alpha}(\varepsilon)(\boldsymbol{v})\right\|_{\mathrm{L}^{2}(\mathcal{Q})}^{2}+\left\|\tilde{e}_{\beta}^{3}(\varepsilon)(\boldsymbol{v})\right\|_{\mathrm{L}^{2}(\mathcal{Q})}^{2}+\left\|\tilde{e}_{3}^{3}(\varepsilon)(\boldsymbol{v})\right\|_{\mathrm{L}^{2}(\mathcal{Q})}^{2}\right)
$$

where $\tilde{e}_{j}^{i}(\varepsilon)$ is the deformation tensor (6.17a)-(6.17c) scaled on $\mathcal{Q}$.

(i) Using (6.17a)-(6.17c) and (6.19), we obtain

$$
\begin{aligned}
\left\|\tilde{e}_{3}^{3}(\varepsilon)\left(\mathrm{v}^{2} \boldsymbol{z}\right)\right\|_{\mathrm{L}^{2}(\mathcal{Q})}^{2} & \lesssim \varepsilon^{-2} \kappa_{1}^{2}|\boldsymbol{\gamma}|_{0 ; S}^{2}, \\
\left\|\tilde{e}_{\sigma}^{3}(\varepsilon)\left(\mathrm{v}^{2} \boldsymbol{z}\right)\right\|_{\mathrm{L}^{2}(\mathcal{Q})}^{2} & \lesssim\left(\varepsilon^{-2}+\kappa_{1}^{2}\right)|\boldsymbol{\gamma}|_{1 ; S}^{2}+\kappa_{2}^{4}|\boldsymbol{\gamma}|_{0 ; S}^{2} \\
\left\|\tilde{e}_{\beta}^{\alpha}(\varepsilon)\left(\mathrm{v}^{2} \boldsymbol{z}\right)\right\|_{\mathrm{L}^{2}(\mathcal{Q})}^{2} & \lesssim\left(|\boldsymbol{\gamma}|_{2 ; S}^{(\boldsymbol{b})}\right)^{2},
\end{aligned}
$$

provided that $\varepsilon$ is sufficiently small $\left(\varepsilon<\kappa_{1}^{-1}=R\right.$ ) to ensure the convergence of the series in $(6.17 \mathrm{c})$. Hence we have

$$
\widetilde{\mathrm{E}}_{3 \mathrm{D}}(\varepsilon)\left[\mathrm{v}^{2} \boldsymbol{z}\right] \lesssim E\left(\varepsilon|\gamma|_{2 ; S}^{2}+\left(\varepsilon^{-1}+\varepsilon \kappa_{1}^{2}\right)|\gamma|_{1 ; S}^{2}+\left(\varepsilon^{-1} \kappa_{1}^{2}+\varepsilon \kappa_{2}^{4}\right)|\gamma|_{0 ; S}^{2}\right)
$$

Multiplying by $\varepsilon^{4}$ and using (5.8), we find

$$
\widetilde{\mathrm{E}}_{3 \mathrm{D}}(\varepsilon)\left[\varepsilon^{2} \mathrm{v}^{2} \boldsymbol{z}\right] \lesssim\left(\varepsilon^{4} L^{-4}+\left(\varepsilon^{2}+\varepsilon^{4} \kappa^{2}\right) L^{-2}+\varepsilon^{2} \kappa^{2}+\varepsilon^{4} \kappa^{4}\right) \mathrm{E}_{2 \mathrm{D}}^{\varepsilon}[\boldsymbol{z}]
$$

with $L=L_{4}$ and $\kappa=\kappa_{5}$, and thus we have

$$
\widetilde{\mathrm{E}}_{3 \mathrm{D}}(\varepsilon)\left[\varepsilon^{2} \mathrm{v}^{2} \boldsymbol{z}\right] \lesssim B_{S}^{1}(\varepsilon ; \boldsymbol{z}) \mathrm{E}_{2 \mathrm{D}}^{\varepsilon}[\boldsymbol{z}]
$$

where $B_{S}^{1}(\varepsilon ; \boldsymbol{z})$ is given in (6.2).

(ii) We recall that the operator $\mathrm{V}^{3}$ is $\boldsymbol{b}$-homogeneous of order 3 and that we have the bound (6.12) for the orders of the derivatives of $\mathrm{V}^{3}$. We deduce that

$$
\begin{aligned}
\left\|\tilde{e}_{3}^{3}(\varepsilon)\left(\mathrm{V}^{3} \boldsymbol{z}\right)\right\|_{\mathrm{L}^{2}(\mathcal{Q})}^{2} & \lesssim \varepsilon^{-2}\left(\left|z_{\alpha}\right|_{3 ; S}+\sum_{j=0}^{2} \kappa_{3}^{3-j}|\boldsymbol{z}|_{j ; S}\right)^{2} \\
\left\|\tilde{e}_{\alpha}^{3}(\varepsilon)\left(\mathrm{V}^{3} \boldsymbol{z}\right)\right\|_{\mathrm{L}^{2}(\mathcal{Q})}^{2} & \lesssim \varepsilon^{-2}\left(\left|z_{3}\right|_{3 ; S}+\sum_{j=0}^{2} \kappa_{3}^{3-j}|\boldsymbol{z}|_{j ; S}\right)^{2}+\left(\left|z_{\alpha}\right|_{4 ; S}+\sum_{j=0}^{3} \kappa_{4}^{4-j}|\boldsymbol{z}|_{j ; S}\right)^{2} \\
\left\|\tilde{e}_{\beta}^{\alpha}(\varepsilon)\left(\mathrm{V}^{3} \boldsymbol{z}\right)\right\|_{\mathrm{L}^{2}(\mathcal{Q})}^{2} & \lesssim\left(\left|z_{3}\right|_{4 ; S}+\sum_{j=0}^{3} \kappa_{4}^{4-j}|\boldsymbol{z}|_{j ; S}\right)^{2} .
\end{aligned}
$$


Hence we have

$$
\widetilde{\mathrm{E}}_{3 \mathrm{D}}(\varepsilon)\left[\mathrm{V}^{3} \boldsymbol{z}\right] \lesssim E \varepsilon^{-1}\left(\sum_{j=0}^{3} \kappa_{3}^{3-j}|\boldsymbol{z}|_{j ; S}\right)^{2}+E \varepsilon\left(\sum_{j=0}^{4} \kappa_{4}^{4-j}|\boldsymbol{z}|_{j ; S}\right)^{2} .
$$

Multiplying by $\varepsilon^{6}$ and using (5.9) we find

$$
\widetilde{\mathrm{E}}_{3 \mathrm{D}}(\varepsilon)\left[\varepsilon^{3} \mathrm{~V}^{3} \boldsymbol{z}\right] \lesssim\left(\sum_{i, j, k \in G_{3}} \frac{\varepsilon^{2 k+2} \kappa_{3}^{2 i}}{L_{2}^{2 j}}+\sum_{i, j, k \in G_{4}} \frac{\varepsilon^{2 k+4} \kappa_{4}^{2 i}}{L_{3}^{2 j}}\right) \mathrm{E}_{2 \mathrm{D}}^{\varepsilon}[\boldsymbol{z}],
$$

where for all $n, G_{n}=\left\{(i, j, k) \in \mathbb{N}^{3} \mid k \in\{0,1\}, i+j=k+n-2\right\}$. We can write the previous equation as

$$
\widetilde{\mathrm{E}}_{3 \mathrm{D}}(\varepsilon)\left[\varepsilon^{3} \mathrm{~V}^{3} \boldsymbol{z}\right] \lesssim\left(\sum_{i, j, k \in G} \frac{\varepsilon^{2 k} \kappa^{2 i}}{L^{2 j}}\right) \mathrm{E}_{2 \mathrm{D}}^{\varepsilon}[\boldsymbol{z}],
$$

where $G=\left\{(i, j, k) \in \mathbb{N}^{3} \mid k \in\{1,2,3\}, i+j=k\right\}$. This shows that

$$
\widetilde{\mathrm{E}}_{3 \mathrm{D}}^{\varepsilon}\left[\varepsilon^{3} \mathrm{~V}^{3} \boldsymbol{z}\right] \lesssim B_{S}^{1}(\varepsilon ; \boldsymbol{z}) \mathrm{E}_{2 \mathrm{D}}^{\varepsilon}[\boldsymbol{z}]
$$

where $B_{S}^{1}(\varepsilon ; \boldsymbol{z})$ is given in (6.2).

(iii) In the same way, we easily find :

$$
\widetilde{\mathrm{E}}_{3 \mathrm{D}}(\varepsilon)\left[\mathrm{V}^{4} \boldsymbol{z}\right] \lesssim E \varepsilon^{-1}\left(\sum_{j=0}^{4} \kappa_{4}^{4-j}|\boldsymbol{z}|_{j ; S}\right)^{2}+E \varepsilon\left(\sum_{j=0}^{5} \kappa_{5}^{5-j}|\boldsymbol{z}|_{j ; S}\right)^{2}
$$

whence the result after multiplying by $\varepsilon^{8}$ and using (5.9). Note that we used $\kappa_{5}$ and $L_{4}$ to obtain this result.

\section{ESTIMATE FOR THE CORRECTOR TERM}

The goal of this section is to construct a displacement $\boldsymbol{u}^{\text {cor }}$ satisfying the equation (3.2) with $B_{2} \lesssim B_{S}\left(\varepsilon, \boldsymbol{z}, \boldsymbol{f}^{\text {rem }}\right)$, and such that $\mathrm{U} \boldsymbol{z}+\boldsymbol{u}^{\text {cor }} \in V\left(\Omega^{\varepsilon}\right)$. In shifted displacements, this amounts to construct $\boldsymbol{w}^{\text {cor }}$ such that $\mathrm{W} \boldsymbol{z}+\boldsymbol{w}^{\text {cor }}$ satisfies lateral Dirichlet conditions and satisfying the same estimates

We recall from Definition 2.7 that $r$ is the geodesic distance to $\partial S$ in $S, s$ the arclength along $\partial S$, and $\mathbf{d}$ defines the tubular neighborhood $(r, s) \in[0, \mathrm{~d}] \times \partial S$. We introduce the adimensional variable $T=r / \varepsilon$. Let $\chi(T)$ be an adimensional $\mathscr{C}^{\infty}$ cut-off function defined on $[0, \infty)$ satisfying $\chi(T) \equiv 1$ for $T \in\left[0, \frac{1}{2}\right]$ and $\chi(T) \equiv 0$ for all $T \geq 1$.

Consider now the displacement $\mathbf{W} \boldsymbol{z}$ as defined in (4.3). As $\boldsymbol{z}$ satisfies the boundary conditions $\left.\boldsymbol{z}\right|_{\partial S}=0$ and $\left.\partial_{r} z_{3}\right|_{\partial S}=0$ we have that $\left.\mathbf{W}_{\alpha} \boldsymbol{z}\right|_{\Gamma_{0}^{\varepsilon}}=0$ and

$$
\left.\mathrm{W}_{3} \boldsymbol{z}\right|_{\Gamma_{0}^{\varepsilon}}=-\left.p x_{3} \gamma_{\alpha}^{\alpha}\right|_{\partial S}+\left.p \frac{x_{3}^{2}}{2} \rho_{\alpha}^{\alpha}\right|_{\partial S}
$$


We thus set

$$
w_{\alpha}^{\mathrm{cor}}=0 \quad \text { and } \quad w_{3}^{\mathrm{cor}}=\chi\left(\varepsilon^{-1} r\right)\left(-\left.p x_{3} \gamma_{\alpha}^{\alpha}\right|_{\partial S}+\left.p \frac{x_{3}^{2}}{2} \rho_{\alpha}^{\alpha}\right|_{\partial S}\right) .
$$

Note that this term is non zero only in the region where $r \leq \varepsilon$. By definition, we have that $\boldsymbol{w}+\boldsymbol{w}^{\text {cor }} \in V\left(\Omega^{\varepsilon}\right)$. It remains to estimate the energy of $\boldsymbol{w}^{\text {cor }}$.

Proposition 7.1 Let $\boldsymbol{w}^{\mathrm{cor}}$ defined by the equation (7.1), then we have the estimate

$$
\widetilde{E}_{3 \mathrm{D}}^{\varepsilon}\left[\boldsymbol{w}^{\mathrm{cor}}\right] \lesssim E \varepsilon^{2}\left(|\boldsymbol{\gamma}|_{0 ; \partial S}^{2}+\varepsilon^{2}|\boldsymbol{\rho}|_{0 ; \partial S}^{2}\right)\left(1+\frac{\varepsilon^{2}}{R^{2}}\right)+E \varepsilon^{4}\left(|\boldsymbol{\gamma}|_{1 ; \partial S}^{2}+\varepsilon^{2}|\boldsymbol{\rho}|_{1 ; \partial S}^{2}\right)
$$

where $R=\kappa_{1}^{-1}$.

Using the definitions of $\ell$ and the fact that $\varepsilon<R$, this estimate proves that

$$
\widetilde{E}_{3 \mathrm{D}}^{\varepsilon}\left[\boldsymbol{w}^{\mathrm{cor}}\right] \lesssim \breve{B}_{S}(\varepsilon ; \boldsymbol{z}) \mathrm{E}_{2 \mathrm{D}}^{\varepsilon}[\boldsymbol{z}] \quad \text { with } \quad \breve{B}_{S}(\varepsilon ; \boldsymbol{z})=\frac{\varepsilon}{\ell}\left(1+\frac{\varepsilon^{2}}{\ell^{2}}\right)
$$

and this finally yields (2.24) provided that $\varepsilon / \ell \leq M$.

Proof of Proposition 7.1. Using the fact that only the transverse component of $\boldsymbol{w}^{\text {cor }}$ is non zero, we have using (6.17a)-(6.17c) that

$$
\widetilde{\mathrm{E}}_{3 \mathrm{D}}^{\varepsilon}\left[\boldsymbol{w}^{\mathrm{cor}}\right] \lesssim E\left\|\partial_{3} w_{3}^{\mathrm{cor}}\right\|_{\mathrm{L}^{2}\left(\Omega^{\varepsilon}\right)}^{2}+E\left\|\mathrm{D}_{\sigma} w_{3}^{\mathrm{cor}}\right\|_{\mathrm{L}^{2}\left(\Omega^{\varepsilon}\right)}^{2}+E \frac{1}{R^{2}}\left\|w_{3}^{\mathrm{cor}}\right\|_{\mathrm{L}^{2}\left(\Omega^{\varepsilon}\right)}^{2} .
$$

Let us recall that in the coordinate system $(r, s)$ in a tubular neighborhood of $\partial S$, the metric satisfies $a_{r r}(r, s)=1, a_{r s}(r, s)=0$, and $a_{s s}(0, s)=1$. This implies that the Euclidean volume form on the tubular neighbourhood $\left\{r \in(0, \mathrm{~d}), s \in \partial S, x_{3} \in(-\varepsilon, \varepsilon)\right\}$ can be written

$$
\mathrm{d} r \mathrm{~d} s \mathrm{~d} x_{3}\left(1+j\left(r, s, x_{3}\right)\right)
$$

where $j\left(r, s, x_{3}\right)$ is an adimensional convergent power series in $r$ and $x_{3}$ provided $r<\mathrm{d}$ and $\left|x_{3}\right|<R$, satisfying $j(0, s, 0)=0$ and with function coefficients defined on $\partial S$. For $r \in(0, \varepsilon), s \in \partial S$ and $x_{3} \in(-\varepsilon, \varepsilon)$, we can always assume for instance (compare (2.2))

$$
\left|1+j\left(r, s, x_{3}\right)\right| \leq 3 / 2 .
$$

Let us decompose $w_{3}^{\text {cor }}=\Phi \boldsymbol{z}+\Psi \boldsymbol{z}$ where

$$
\Phi \boldsymbol{z}=-\left.p x_{3} \gamma_{\alpha}^{\alpha}\right|_{\partial S} \chi\left(\varepsilon^{-1} r\right) \quad \text { and } \quad \Psi \boldsymbol{z}=\left.p \frac{x_{3}^{2}}{2} \rho_{\alpha}^{\alpha}\right|_{\partial S} \chi\left(\varepsilon^{-1} r\right)
$$

We see that

$$
\|\Phi \boldsymbol{z}\|_{L^{2}\left(\Omega^{\varepsilon}\right)}^{2}=p^{4} \varepsilon^{4} \int_{-1}^{1} \int_{\partial S} \int_{0}^{1} X_{3}^{2} \chi(\varepsilon T)^{2}\left(\left.\gamma_{\alpha}^{\alpha}(\boldsymbol{z})\right|_{\partial S}\right)^{2}\left|1+j\left(\varepsilon T, s, \varepsilon X_{3}\right)\right| \mathrm{d} T \mathrm{~d} s \mathrm{~d} X_{3} .
$$

This implies immediately that $\|\Phi \boldsymbol{z}\|_{\mathrm{L}^{2}\left(\Omega^{\varepsilon}\right)}^{2} \lesssim \varepsilon^{4}|\gamma|_{0 ; \partial S}^{2}$. We have a similar estimate for $\Psi$. Hence we deduce

$$
\left\|w_{3}^{\mathrm{cor}}\right\|_{\mathrm{L}^{2}\left(\Omega^{\varepsilon}\right)}^{2} \lesssim \varepsilon^{4}|\gamma|_{0 ; \partial S}^{2}+\varepsilon^{6}|\boldsymbol{\rho}|_{0 ; \partial S}^{2}
$$


Similarly, we easily see that

$$
\left\|\partial_{3} w_{3}^{\text {cor }}\right\|_{L^{2}\left(\Omega^{\varepsilon}\right)}^{2} \lesssim \varepsilon^{2}|\gamma|_{0 ; \partial S}^{2}+\varepsilon^{4}|\boldsymbol{\rho}|_{0 ; \partial S}^{2}
$$

Note that with the change of coordinate $\left(r, s, x_{3}\right) \mapsto\left(T, s, X_{3}\right)$, the term $\left\|\mathrm{D}_{\sigma} w_{3}^{\text {cor }}\right\|_{\mathrm{L}^{2}\left(\Omega^{\varepsilon}\right)}^{2}$ has to be understood as (see formula (A.4))

$$
\int_{\Omega^{\varepsilon}} a^{\alpha \beta}\left(x_{3}\right)\left(\mathrm{D}_{\alpha} w_{3}^{\text {cor }}\right)\left(\mathrm{D}_{\beta} w_{3}^{\text {cor }}\right) \mathrm{d} V
$$

As $\varepsilon \leq \varepsilon_{0}<\mathrm{d}$, we can assume that the metric on $S$ in coordinates $(r, s)$ is $\mathcal{O}(\varepsilon / \mathrm{d})$ close to the identity. So, for $r \in(0, \varepsilon), s \in \partial S$ and $x_{3} \in(-\varepsilon, \varepsilon)$ with $\varepsilon<\min (R, \mathrm{~d})$, this yields

$$
\left\|\mathrm{D}_{\sigma} w_{3}^{\mathrm{cor}}\right\|_{\mathrm{L}^{2}\left(\Omega^{\varepsilon}\right)}^{2} \lesssim \int_{0}^{\varepsilon} \int_{\partial S} \int_{-\varepsilon}^{\varepsilon}\left(\left(\partial_{r} w_{3}^{\mathrm{cor}}\right)^{2}+\left(\partial_{s} w_{3}^{\mathrm{cor}}\right)^{2}\right) \mathrm{d} r \mathrm{~d} s \mathrm{~d} x_{3} .
$$

But we have

$$
\partial_{s} \Phi \boldsymbol{z}=-\left.p x_{3}\left(\partial_{s} \gamma_{\alpha}^{\alpha}\right)\right|_{\partial S} \chi\left(\varepsilon^{-1} r\right)
$$

and

$$
\partial_{r} \Phi \boldsymbol{z}=-\left.\varepsilon^{-1} p x_{3} \gamma_{\alpha}^{\alpha}\right|_{\partial S}\left(\partial_{T} \chi\right)\left(\varepsilon^{-1} r\right)
$$

where here $\partial_{T} \chi$ is an adimensional function with support in $T \in(0,1)$. This shows that

$$
\left\|\mathrm{D}_{\sigma} \Phi \boldsymbol{z}\right\|_{\mathrm{L}^{2}\left(\Omega^{\varepsilon}\right)}^{2} \lesssim \varepsilon^{2}|\gamma|_{0 ; \partial S}^{2}+\varepsilon^{4}|\gamma|_{1 ; \partial S}^{2}
$$

and similarly

$$
\left\|\mathrm{D}_{\sigma} \Psi \boldsymbol{z}\right\|_{\mathrm{L}^{2}\left(\Omega^{\varepsilon}\right)}^{2} \lesssim \varepsilon^{4}|\boldsymbol{\rho}|_{0 ; \partial S}^{2}+\varepsilon^{6}|\boldsymbol{\rho}|_{1 ; \partial S}^{2} .
$$

Collecting together the previous estimates yields the result.

\section{CONCLUSiON: OPTIMALITY OF THE MAIN ESTIMATE}

To conclude our paper, we apply estimate (2.24) to families $\left(\boldsymbol{u}^{\varepsilon}\right)$ and $\left(\boldsymbol{z}^{\varepsilon}\right)$ of solutions of problems $\left(P_{3 \mathrm{D}}\right)$ and $\left(P_{2 \mathrm{D}}\right)$ for each $\varepsilon \in\left(0, \varepsilon_{0}\right]$ for a smooth fixed "standard load" $\boldsymbol{f}$, which means that $\boldsymbol{f}$ has the form $\boldsymbol{f}\left(x_{\alpha}, x_{3}\right)=\boldsymbol{g}\left(x_{\alpha}\right)$ with a smooth surface load $\boldsymbol{g}$ independent of $\varepsilon$. Hence $\boldsymbol{g}$ is the mean value (2.11) of $\boldsymbol{f}$ across the shell $\Omega^{\varepsilon}$.

\section{A Plates}

A family of plates $\left(\Omega^{\varepsilon}\right)$ is defined by its mid-surface $S$ which is a domain of $\mathbb{R}^{2}$. Thus the normal coordinates $\left(x_{\alpha}, x_{3}\right)$ are globally defined by a Cartesian coordinate system. Hence, the metric is the flat metric and the curvature vanishes on $S$. Consequently, we have $\kappa_{j}=0$ for all $j \geq 1$, thus $r=\infty$. Moreover the membrane and change of curvature tensor reduce to

$$
\gamma_{\alpha \beta}(\boldsymbol{z})=\frac{1}{2}\left(\partial_{\alpha} z_{\beta}+\partial_{\beta} z_{\alpha}\right) \quad \text { and } \quad \rho_{\alpha \beta}(\boldsymbol{z})=\partial_{\alpha \beta} z_{3} .
$$


This shows that the Koiter operator decouples into the restrictions $M_{*}$ and $B_{3}$ of the membrane and bending operators acting on the surfacic and transverse components $\boldsymbol{z}_{*}$ and $z_{3}$ respectively:

$$
\mathrm{K}(\varepsilon)=\left(\begin{array}{cc}
\mathrm{M}_{*} & 0 \\
0 & \varepsilon^{2} \mathrm{~B}_{3}
\end{array}\right)
$$

Thus the solution of the problem $\left(P_{2 \mathrm{D}}\right)$ is given by

$$
\boldsymbol{z}^{\varepsilon}=\left(\boldsymbol{z}_{\mathrm{M}}, 0\right)+\varepsilon^{-2}\left(0, z_{\mathrm{B}}\right)
$$

where the membrane and bending parts $z_{\mathrm{M}} \in \mathbf{H}_{0}^{1}(S)$ and $z_{\mathrm{B}} \in \mathrm{H}_{0}^{2}(S)$ solve the equations $\mathrm{M}_{*} z_{\mathrm{M}}=\boldsymbol{g}_{*}$ and $\mathrm{B}_{3} z_{\mathrm{B}}=g_{3}$. Hence the wave lengths $L$ and $\ell$ associated with $\boldsymbol{z}^{\varepsilon}$ are in fact independent on $\varepsilon$.

Estimate (2.24) with $\boldsymbol{f}^{\mathrm{rem}}=\mathbf{0}$ then yields

$$
\mathrm{E}_{3 \mathrm{D}}^{\varepsilon}\left[\boldsymbol{u}^{\varepsilon}-\mathrm{U} \boldsymbol{z}^{\varepsilon}\right] \leq b_{S}(\boldsymbol{g}) \varepsilon \mathrm{E}_{2 \mathrm{D}}^{\varepsilon}\left[\boldsymbol{z}^{\varepsilon}\right]
$$

where $b_{S}(\boldsymbol{g})$ has the dimension of the inverse of a length.

In [16], it is shown that the displacement $\boldsymbol{u}^{\varepsilon}$ admits a complete two scale asymptotic expansion in powers of $\varepsilon$. This expansion includes regular terms bounded independently of $\varepsilon$, and boundary layer term exponentially decreasing with respect to $r / \varepsilon$ where $r$ is the distance to $\partial S$. Relying on this result, we can prove that the following optimal estimates holds true, see $[16, \S 12.2]$ :

$$
\begin{aligned}
& b_{S}^{\prime}(\boldsymbol{g}) \varepsilon \mathrm{E}_{3 \mathrm{D}}^{\varepsilon}\left[\boldsymbol{u}^{\varepsilon}\right] \leq \mathrm{E}_{3 \mathrm{D}}^{\varepsilon}\left[\boldsymbol{u}^{\varepsilon}-\mathrm{U} \boldsymbol{z}^{\varepsilon}\right] \leq b_{S}(\boldsymbol{g}) \varepsilon \mathrm{E}_{3 \mathrm{D}}^{\varepsilon}\left[\boldsymbol{u}^{\varepsilon}\right] \\
& a_{S}^{\prime}(\boldsymbol{g}) \mathrm{E}_{2 \mathrm{D}}^{\varepsilon}\left[\boldsymbol{z}^{\varepsilon}\right] \leq \mathrm{E}_{3 \mathrm{D}}^{\varepsilon}\left[\boldsymbol{u}^{\varepsilon}\right] \leq a_{S}(\boldsymbol{g}) \mathrm{E}_{2 \mathrm{D}}^{\varepsilon}\left[\boldsymbol{z}^{\varepsilon}\right],
\end{aligned}
$$

where $b_{S}(\boldsymbol{g}), b_{S}^{\prime}(\boldsymbol{g})$ have the dimension of the inverse of a length and $a_{S}(\boldsymbol{g}), a_{S}^{\prime}(\boldsymbol{g})$ are adimensional. In relation with the generic non-cancellation of the traces of $\gamma_{\alpha}^{\alpha}=\operatorname{div} z_{\mathrm{M}}$ or $\rho_{\alpha}^{\alpha}=\Delta z_{\mathrm{B}}$, the constant $b_{S}^{\prime}(\boldsymbol{g})$ is generically non-zero. This shows how (8.1) is optimal in the case of plates.

\section{B ELLiptic SHELLS}

In the case of elliptic shells, the curvature tensor $b_{\alpha \beta}$ satisfies an estimate of the form $b_{\alpha \beta} \xi^{\alpha} \xi^{\beta} \geq c \xi^{\alpha} \xi_{\alpha}$ for all vector field $\xi^{\alpha}$ on $S$ and for a uniform constant $c$ independent on $\xi$. This implies that the constant $r$ is a positive number.

Using the result in [20], it is possible to estimate the behaviour of the constants $L$ and $\ell$ with $\varepsilon$. In [20], it is shown that $\boldsymbol{z}^{\varepsilon}$ admit a multi-scale asymptotic expansion

$$
\boldsymbol{z}^{\varepsilon} \simeq \boldsymbol{\zeta}^{0}+\varepsilon^{1 / 2}\left(\boldsymbol{Z}^{1 / 2}+\boldsymbol{\zeta}^{1 / 2}\right)+\varepsilon\left(\boldsymbol{Z}^{1}+\boldsymbol{\zeta}^{1}\right) \cdots
$$

in powers of $\varepsilon^{1 / 2}$, where the regular terms $\zeta^{k / 2}$ are uniformly bounded in $\varepsilon$, and where the terms $Z^{k / 2}$ are boundary layer terms. These terms are sums of functions that are tensor products of smooth functions of $s \in \partial S$ and exponentially decreasing functions with respect to the variable $r \sqrt{b_{s s}} / \sqrt{\varepsilon}$ where $b_{s s}$ is the (non zero) curvature along the boundary $\partial S$ (see 
equation (1.12) in [20]). This shows that $L \simeq\left(\varepsilon R_{\partial}\right)^{1 / 2}$ where $R_{\partial}$ denote the maximum of curvature radius along the boundary $\partial S$, and that $\ell$ is a positive constant independent of $\varepsilon$. Hence, estimate (2.24) with $f^{\text {rem }}=\mathbf{0}$ yields as before

$$
\mathrm{E}_{3 \mathrm{D}}^{\varepsilon}\left[\boldsymbol{u}^{\varepsilon}-\mathrm{U} \boldsymbol{z}^{\varepsilon}\right] \leq b_{S}(\boldsymbol{g}) \varepsilon \mathrm{E}_{2 \mathrm{D}}^{\varepsilon}\left[\boldsymbol{z}^{\varepsilon}\right]
$$

As in the case of plates, this estimate turns to be optimal, see Theorem 1.4 in [20].

\section{C Shallow Shells}

Shallow shells in the sense of [13] are shells for which the mid-surface $S=S^{\varepsilon}$ depend on $\varepsilon$ in such a way that the curvature tensor is of order $B \varepsilon$ where $B$ has now the dimension $m^{-2}$. The limit surface $S^{0}$ is a domain of $\mathbb{R}^{2}$. The constant $r$ is hence of order $(B \varepsilon)^{-1}$. Denote by $\left(x_{\alpha}^{\varepsilon}, x_{3}^{\varepsilon}\right)$ normal coordinates to $S^{\varepsilon}$, for $\varepsilon \in\left[0, \varepsilon_{0}\right]$.

We define a regular family of loads $\boldsymbol{f}^{\varepsilon}$ on $\Omega^{\varepsilon}$ in the following way. For a fixed smooth surface load $\boldsymbol{G}$ given in a neighborhood of $S^{0}$ in $\mathbb{R}^{2}$, we define the field $\boldsymbol{F}$ by $\boldsymbol{F}\left(x_{\alpha}^{0}, x_{3}^{0}\right)=\boldsymbol{G}\left(x_{\alpha}^{0}\right)$. Then we set

$$
\boldsymbol{f}^{\varepsilon}=\left.\boldsymbol{F}\right|_{\Omega^{\varepsilon}} \quad \text { and } \quad \boldsymbol{g}^{\varepsilon}=\frac{1}{2 \varepsilon} \int_{-\varepsilon}^{\varepsilon} \boldsymbol{f}^{\varepsilon}\left(x_{3}^{\varepsilon}\right) \mathrm{d} x_{3}^{\varepsilon},
$$

and $\boldsymbol{f}^{\varepsilon}$ is the load in problem $\left(P_{3 \mathrm{D}}\right)$ on $\Omega^{\varepsilon}$, while $\boldsymbol{g}^{\varepsilon}$ is the right hand side of problem $\left(P_{2 \mathrm{D}}\right)$ on $S^{\varepsilon}$.

In this situation, the Koiter model can be seen as an operator which couples the membrane and bending operators for plates through low order terms, and it can be shown that $\boldsymbol{z}^{\varepsilon}$ admits a complete asymptotic expansion in powers of $\varepsilon$ with regular terms only. Hence, the constant $\ell$ and $L$ are independent of $\varepsilon$, and estimate (2.24) yields an estimate similar to (8.1).

In [4], it has been shown that the three dimensional displacement $\boldsymbol{u}^{\varepsilon}$ admits a complete asymptotic expansion in powers of $\varepsilon$ with regular bounded terms and boundary layer terms exponentially decreasing in $r / \varepsilon$. Using this result, it can again be shown that estimates of the form (8.2) hold true in the case of shallow shells. This shows that (2.24) is optimal in this case.

\section{APPENDiX A ESTIMATE FOR THE DIFFERENCE OF ENERGIES}

We give here an estimate between the energy of a two-dimensional displacement $z$ and the three-dimensional energy of the reconstructed displacement $\mathrm{U} z$ :

Theorem A.1 For all $\boldsymbol{z} \in\left(\mathbf{H}^{2} \cap \mathbf{H}_{0}^{1}\right)(S) \times\left(\mathrm{H}^{3} \cap \mathrm{H}_{0}^{2}\right)(S)$, we have the following estimate

$$
\left|\mathrm{E}_{2 \mathrm{D}}^{\varepsilon}[\boldsymbol{z}]-\mathrm{E}_{3 \mathrm{D}}^{\varepsilon}[\mathrm{U} \boldsymbol{z}]\right| \leq A\left(\frac{\varepsilon}{R}+\frac{\varepsilon^{2}}{L_{1}^{2}}\right) \mathrm{E}_{2 \mathrm{D}}^{\varepsilon}[\boldsymbol{z}],
$$

for an adimensional constant $A$, where $L_{1}$ is the first wave length for $z$ defined according to Definition 2.4, and $R=\kappa_{1}^{-1}$ according to Definition 2.1. 


\section{Proof.}

STEP 1. The proof is easier when using the shifted displacement $W z$, see (4.3), corresponding to the reconstructed displacement $U \boldsymbol{z}$. For any three-dimensional displacement $\boldsymbol{u}$, we recall that $E_{3 \mathrm{D}}^{\varepsilon}[\boldsymbol{u}]$ denotes its quadratic energy, cf. (2.5). If $\boldsymbol{w}$ is the shifted displacement associated with $\boldsymbol{u}$ we denote the corresponding energy by $\widetilde{\mathrm{E}}_{3 \mathrm{D}}^{\varepsilon}[\boldsymbol{w}]$ which is defined so that $\widetilde{\mathrm{E}}_{3 \mathrm{D}}^{\varepsilon}[\boldsymbol{w}]=\mathrm{E}_{3 \mathrm{D}}^{\varepsilon}[\boldsymbol{u}]$. Hence we have

$$
\widetilde{\mathrm{E}}_{3 \mathrm{D}}^{\varepsilon}[\boldsymbol{w}]=\int_{\Omega^{\varepsilon}} A^{i j k \ell} \tilde{e}_{i j}(\boldsymbol{w}) \tilde{e}_{k \ell}(\boldsymbol{w}) \mathrm{d} V
$$

where the modified strain tensor $\tilde{e}_{i j}(\boldsymbol{w})$ is defined so that $\tilde{e}_{i j}(\boldsymbol{w})=e_{i j}(\boldsymbol{u})$. In normal coordinates we have the following expressions for the tensor $\tilde{e}_{i j}(\boldsymbol{w})$, see [19] and (6.17a)(6.17c):

$$
\begin{aligned}
\tilde{e}_{3}^{3}(\boldsymbol{w}) & =\partial_{x_{3}} w_{3}, \\
\tilde{e}_{\beta}^{3}(\boldsymbol{w}) & =\frac{1}{2}\left(\partial_{x_{3}} w_{\beta}-x_{3} b_{\beta}^{\alpha} \partial_{x_{3}} w_{\alpha}+\theta_{\beta}(\boldsymbol{w})\right) \\
\tilde{e}_{\beta}^{\alpha}(\boldsymbol{w}) & =\gamma_{\beta}^{\alpha}(\boldsymbol{w})+\sum_{n=1}^{\infty} x_{3}^{n}\left(b^{n}\right)_{\delta}^{\alpha} \gamma_{\beta}^{\delta}(\boldsymbol{w})+\sum_{n=1}^{\infty} n x_{3}^{n}\left(b^{n-1}\right)_{\delta}^{\alpha} \Lambda_{\cdot \beta}^{\delta}(\boldsymbol{w}),
\end{aligned}
$$

where $\theta_{\beta}(\boldsymbol{z})=\mathrm{D}_{\beta} z_{3}+b_{\beta}^{\alpha} z_{\alpha}$ and $\Lambda_{\alpha \beta}(\boldsymbol{z})=\frac{1}{2}\left(b_{\alpha}^{\sigma} \mathrm{D}_{\sigma} z_{\beta}-b_{\beta}^{\sigma} \mathrm{D}_{\alpha} z_{\sigma}\right)$.

Using the definition of the rigidity tensor, we obtain

$$
\begin{aligned}
\widetilde{\mathrm{E}}_{3 \mathrm{D}}^{\varepsilon}[\boldsymbol{w}]=\int_{\Omega^{\varepsilon}}[(\lambda+2 \mu) & \tilde{e}_{3}^{3}(\boldsymbol{w}) \tilde{e}_{3}^{3}(\boldsymbol{w})+2 \lambda \tilde{e}_{3}^{3}(\boldsymbol{w}) \tilde{e}_{\alpha}^{\alpha}(\boldsymbol{w})+\lambda \tilde{e}_{\alpha}^{\alpha}(\boldsymbol{w}) \tilde{e}_{\beta}^{\beta}(\boldsymbol{w}) \\
& \left.+4 \mu a^{\alpha \beta}\left(x_{3}\right) \tilde{e}_{\alpha}^{3}(\boldsymbol{w}) \tilde{e}_{\beta}^{3}(\boldsymbol{w})+2 \mu \tilde{e}_{\alpha}^{\beta}(\boldsymbol{w}) \tilde{e}_{\beta}^{\alpha}(\boldsymbol{w})\right] \mathrm{d} t^{1} \mathrm{~d} t^{2} \mathrm{~d} t^{3},
\end{aligned}
$$

where $a^{\alpha \beta}\left(x_{3}\right)=a^{\sigma \nu}\left(\mu^{-1}\left(x_{3}\right)\right)_{\sigma}^{\alpha}\left(\mu^{-1}\left(x_{3}\right)\right)_{\nu}^{\beta}$ is the inverse of the metric tensor of the surface at the level $x_{3}$ in the shell, see $[32,19]$. We thus reduce the proof to showing that $\left|\tilde{E}_{3 \mathrm{D}}^{\varepsilon}[\mathrm{W} \boldsymbol{z}]-\mathrm{E}_{2 \mathrm{D}}^{\varepsilon}[\boldsymbol{z}]\right|$ is bounded by the right hand side (A.1). We note that $\mathrm{E}_{2 \mathrm{D}}^{\varepsilon}[\boldsymbol{z}]$ is associated with the material law of Lamé coefficients $2 \mu p$ and $\mu$ (we recall that $p=\lambda(\lambda+2 \mu)^{-1}$ ) and writes

$$
\begin{aligned}
\mathrm{E}_{2 \mathrm{D}}^{\varepsilon}[\boldsymbol{z}]=2 \varepsilon \int_{S}\left[2 \mu p \gamma_{\alpha}^{\alpha}(\boldsymbol{z}) \gamma_{\beta}^{\beta}(\boldsymbol{z})\right. & \left.+2 \mu \gamma_{\alpha}^{\beta}(\boldsymbol{z}) \gamma_{\beta}^{\alpha}(\boldsymbol{z})\right] \mathrm{d} S \\
& +\frac{2}{3} \varepsilon^{3} \int_{S}\left[2 \mu p \rho_{\alpha}^{\alpha}(\boldsymbol{z}) \rho_{\beta}^{\beta}(\boldsymbol{z})+2 \mu \rho_{\alpha}^{\beta}(\boldsymbol{z}) \rho_{\beta}^{\alpha}(\boldsymbol{z})\right] \mathrm{d} S .
\end{aligned}
$$

STEP 2. We are going to calculate each term forming $E_{3 D}^{\varepsilon}[W z]$ with the help of the splitting of $\mathrm{W} z$ into the sum of a displacement of Kirchhoff-Love type $\mathrm{W}^{\mathrm{KL}} \boldsymbol{z}$ and of a complementary term $W^{\mathrm{cmp}} \boldsymbol{z}$ which is a transverse quadratic displacement:

$$
\mathrm{W}^{\mathrm{KL}} \boldsymbol{z}=\left\{\begin{array}{l}
z_{\sigma}-x_{3} \theta_{\sigma}(\boldsymbol{z}), \quad \text { and } \quad \mathrm{W}^{\mathrm{cmp}} \boldsymbol{z}=\left\{\begin{array}{l}
0, \\
z_{3},
\end{array}-x_{3} p \gamma_{\alpha}^{\alpha}(\boldsymbol{z})+\frac{x_{3}^{2}}{2} p \rho_{\alpha}^{\alpha}(\boldsymbol{z}) .\right.
\end{array}\right.
$$


Lemma A.2 With the minimal principal radius of curvature $R=\kappa_{1}^{-1}$, we have:

$$
\begin{aligned}
& \tilde{e}_{i}^{3}\left(\mathbf{W}^{\mathrm{KL}} \boldsymbol{z}\right)=0 \text { for } i=\sigma, 3 \\
& \tilde{e}_{\sigma}^{\alpha}\left(\mathrm{W}^{\mathrm{KL}} \boldsymbol{z}\right)=\gamma_{\sigma}^{\alpha}(\boldsymbol{z})-x_{3}\left(\rho_{\sigma}^{\alpha}(\boldsymbol{z})-2 b_{\delta}^{\alpha} \gamma_{\sigma}^{\delta}(\boldsymbol{z})\right)+\sum_{n=1}^{\infty} x_{3}^{1+n}\left(P_{n}^{\mathrm{KL}}\right)_{\sigma}^{\alpha}(\boldsymbol{z})
\end{aligned}
$$

and

$$
\begin{aligned}
\tilde{e}_{3}^{3}\left(\mathbf{W}^{\mathrm{cmp}} \boldsymbol{z}\right) & =-p \gamma_{\alpha}^{\alpha}(\boldsymbol{z})+p x_{3} \rho_{\alpha}^{\alpha}(\boldsymbol{z}) \\
2 \tilde{e}_{\sigma}^{3}\left(\mathrm{~W}^{\mathrm{cmp}} \boldsymbol{z}\right) & =-x_{3} p \mathrm{D}_{\sigma} \gamma_{\alpha}^{\alpha}(\boldsymbol{z})+\frac{x_{3}^{2}}{2} p \mathrm{D}_{\sigma} \rho_{\alpha}^{\alpha}(\boldsymbol{z}), \\
\tilde{e}_{\sigma}^{\alpha}\left(\mathrm{W}^{\mathrm{cmp}} \boldsymbol{z}\right) & =x_{3} p b_{\sigma}^{\alpha} \gamma_{\delta}^{\delta}(\boldsymbol{z})+\sum_{n=1}^{\infty} x_{3}^{1+n}\left(P_{n}^{\mathrm{cmp}}\right)_{\sigma}^{\alpha}(\boldsymbol{z}),
\end{aligned}
$$

where the tensors $\left(P_{n}^{\mathrm{KL}}\right)(\boldsymbol{z})$ and $\left(P_{n}^{\mathrm{cmp}}\right)(\boldsymbol{z})$ satisfy the estimates, for all $n \geq 1$,

$$
\left|\left(P_{n}^{\mathrm{KL}}\right)(\boldsymbol{z})\right|_{0 ; S}+\left|\left(P_{n}^{\mathrm{cmp}}\right)(\boldsymbol{z})\right|_{0 ; S} \leq \frac{A n}{R^{n}}\left(|\boldsymbol{\rho}(\boldsymbol{z})|_{0 ; S}+\frac{1}{R}|\boldsymbol{\gamma}(\boldsymbol{z})|_{0 ; S}\right)
$$

for an adimensional constant $A$.

Equation (A.6a) justifies the denomination of $\mathrm{W}^{\mathrm{KL}} \boldsymbol{z}$ after Kirchhoff-Love.

Proof. It is clear that $\tilde{e}_{3}^{3}\left(\mathrm{~W}^{\mathrm{KL}} \boldsymbol{z}\right)=\partial_{x_{3}} z_{3}=0$. Using equality (A.3b), we calculate

$$
2 \tilde{e}_{\sigma}^{3}\left(\mathbf{W}^{\mathrm{KL}} \boldsymbol{z}\right)=-\theta_{\sigma}(\boldsymbol{z})+x_{3} b_{\sigma}^{\alpha} \theta_{\alpha}(\boldsymbol{z})+\theta_{\sigma}(\boldsymbol{z})-x_{3} b_{\sigma}^{\alpha} \theta_{\alpha}(\boldsymbol{z})=0,
$$

which yields (A.6a).

The equation (A.7a) is clear. The expression (A.3b) of the operator $\tilde{e}_{\sigma}^{3}(\boldsymbol{w})$ yields (A.7b).

To obtain (A.6b) we first note that

$$
\Lambda_{\alpha \beta}\left(\mathrm{W}^{\mathrm{KL}} \boldsymbol{z}\right)=\Lambda_{\alpha \beta}(\boldsymbol{z})-\frac{x_{3}}{2}\left(b_{\alpha}^{\sigma} \mathrm{D}_{\sigma} \theta_{\beta}(\boldsymbol{z})-b_{\beta}^{\sigma} \mathrm{D}_{\alpha} \theta_{\sigma}(\boldsymbol{z})\right)
$$

and hence as $\mathrm{D}_{\sigma} \theta_{\beta}(\boldsymbol{z})=\rho(\boldsymbol{z})+b_{\sigma}^{\nu} b_{\nu \beta} z_{3}-b_{\sigma}^{\nu} \mathrm{D}_{\beta} z_{\nu}$ we have

$$
\left|\Lambda_{\alpha \beta}\left(\mathrm{W}^{\mathrm{KL}} \boldsymbol{z}\right)\right|_{0 ; S} \leq \frac{1}{R}\left|z_{\sigma}\right|_{1 ; S}+\frac{x_{3}}{R}|\boldsymbol{\rho}(\boldsymbol{z})|_{0 ; S}+\frac{x_{3}}{R^{2}}\left|z_{\sigma}\right|_{1 ; S}+\frac{x_{3}}{R^{3}}\left|z_{3}\right|_{0 ; S}
$$

With expression (A.3c) we compute that

$$
\tilde{e}_{\sigma}^{\alpha}\left(\mathrm{W}^{\mathrm{KL}} \boldsymbol{z}\right)=\gamma_{\sigma}^{\alpha}(\boldsymbol{z})+x_{3} b_{\delta}^{\alpha} \gamma_{\sigma}^{\delta}(\boldsymbol{z})+x_{3} \Lambda_{\cdot \sigma}^{\alpha} \cdot(\boldsymbol{z})-x_{3} \bar{\rho}_{\sigma}^{\alpha}(\boldsymbol{z})+\sum_{n=1}^{\infty} x_{3}^{1+n}\left(P_{n}^{\mathrm{KL}}\right)_{\sigma}^{\alpha}(\boldsymbol{z})
$$

where $\bar{\rho}_{\alpha \beta}=\frac{1}{2}\left(\mathrm{D}_{\alpha} \theta_{\beta}+\mathrm{D}_{\beta} \theta_{\alpha}\right)$ and where the tensors $\left(P_{n}^{\mathrm{KL}}\right)(\boldsymbol{z})$ satisfy the estimate

$$
\left|\left(P_{n}^{\mathrm{KL}}\right)(\boldsymbol{z})\right|_{0 ; S} \leq \frac{A n}{R^{n}}\left(|\boldsymbol{\rho}(\boldsymbol{z})|_{0 ; S}+\frac{1}{R}|\boldsymbol{z}|_{1 ; S}+\frac{1}{R^{2}}|\boldsymbol{z}|_{0 ; S}\right) .
$$

But we have

$$
\bar{\rho}_{\beta}^{\alpha}-\Lambda_{\cdot \beta}^{\alpha \cdot}=\rho_{\beta}^{\alpha}-b_{\sigma}^{\alpha} \gamma_{\beta}^{\sigma}
$$


Moreover, using (5.5) with $n=1$, we have that for all $\boldsymbol{z} \in \mathbf{H}_{0}^{1} \times \mathrm{H}_{0}^{2}(S)$,

$$
\frac{1}{R}|\boldsymbol{z}|_{1 ; S}+\frac{1}{R^{2}}|\boldsymbol{z}|_{0 ; S} \leq A_{1}\left(|\boldsymbol{\rho}(\boldsymbol{z})|_{0 ; S}+\frac{1}{R}|\boldsymbol{\gamma}(\boldsymbol{z})|_{0 ; S}\right) \text {. }
$$

Therefore we get (A.6b). The proof of (A.7c) is similar.

STEP 3. Gathering the previous results and setting $\left(P_{n}\right)(\boldsymbol{z})=\left(P_{n}^{\mathrm{KL}}\right)(\boldsymbol{z})+\left(P_{n}^{\mathrm{cmp}}\right)(\boldsymbol{z})$, we find that

$$
\begin{aligned}
& \tilde{e}_{3}^{3}(\mathbf{W} \boldsymbol{z})=-p \gamma_{\alpha}^{\alpha}(\boldsymbol{z})+p x_{3} \rho_{\alpha}^{\alpha}(\boldsymbol{z}) \\
& \tilde{e}_{\sigma}^{3}(\mathbf{W} \boldsymbol{z})=-\frac{x_{3}}{2} p \mathrm{D}_{\sigma} \gamma_{\alpha}^{\alpha}(\boldsymbol{z})+\frac{x_{3}^{2}}{4} p \mathrm{D}_{\sigma} \rho_{\alpha}^{\alpha}(\boldsymbol{z}), \\
& \tilde{e}_{\sigma}^{\alpha}(\mathbf{W} \boldsymbol{z})=\gamma_{\sigma}^{\alpha}(\boldsymbol{z})-x_{3}\left(\rho_{\sigma}^{\alpha}(\boldsymbol{z})-p b_{\sigma}^{\alpha} \gamma_{\delta}^{\delta}(\boldsymbol{z})-2 b_{\delta}^{\alpha} \gamma_{\sigma}^{\delta}(\boldsymbol{z})\right)+\sum_{n=1}^{\infty} x_{3}^{1+n}\left(P_{n}\right)_{\sigma}^{\alpha}(\boldsymbol{z})
\end{aligned}
$$

where $\left(P_{n}\right)(\boldsymbol{z})$ satisfies the estimate (A.8).

We compute now the different contributions in the integral (A.4). The previous computations yield a convergent series expansion of each term in powers of $x_{3}$. Therefore each contribution in the integral (A.4) has also a convergent series expansion in powers of $x_{3}$. When integrating with respect to $x_{3}$ from $-\varepsilon$ to $\varepsilon$, the odd powers of $x_{3}$ have no contribution. Based on this remark we immediately obtain, first:

$$
\begin{aligned}
\int_{\Omega^{\varepsilon}}(\lambda+2 \mu) \tilde{e}_{3}^{3}(\mathbf{W} \boldsymbol{z}) \tilde{e}_{3}^{3}(\mathbf{W} \boldsymbol{z}) \mathrm{d} t^{1} \mathrm{~d} t^{1} \mathrm{~d} t^{3} & =2 \varepsilon(\lambda+2 \mu) p^{2} \int_{S} \gamma_{\alpha}^{\alpha}(\boldsymbol{z}) \gamma_{\beta}^{\beta}(\boldsymbol{z}) \mathrm{d} S \\
& +\frac{2 \varepsilon^{3}}{3}(\lambda+2 \mu) p^{2} \int_{S} \rho_{\alpha}^{\alpha}(\boldsymbol{z}) \rho_{\beta}^{\beta}(\boldsymbol{z}) \mathrm{d} S+Q_{0}(\varepsilon, \boldsymbol{z})
\end{aligned}
$$

where the term $Q_{0}(\varepsilon, \boldsymbol{z})$ is due to the function $h$ is (2.2) and thus satisfies, using (5.8)

$$
\left|Q_{0}(\varepsilon, \boldsymbol{z})\right| \leq A \frac{\varepsilon}{R} \mathrm{E}_{2 \mathrm{D}}^{\varepsilon}[\boldsymbol{z}]
$$

Then:

$$
\begin{aligned}
\int_{\Omega^{\varepsilon}} 2 \lambda \tilde{e}_{3}^{3}(\mathbf{W} \boldsymbol{z}) \tilde{e}_{\alpha}^{\alpha}(\mathbf{W} \boldsymbol{z}) \mathrm{d} t^{1} \mathrm{~d} t^{1} \mathrm{~d} t^{3} & = \\
& -4 \varepsilon \lambda p \int_{S}\left[\gamma_{\alpha}^{\alpha}(\boldsymbol{z}) \gamma_{\beta}^{\beta}(\boldsymbol{z})+\frac{\varepsilon^{2}}{3} \rho_{\alpha}^{\alpha}(\boldsymbol{z}) \rho_{\beta}^{\beta}(\boldsymbol{z})\right] \mathrm{d} S+Q_{1}(\varepsilon, \boldsymbol{z})
\end{aligned}
$$

where

$$
\begin{aligned}
Q_{1}(\varepsilon, \boldsymbol{z})=\int_{\Omega^{\varepsilon}}( & 2 x_{3}^{2} \lambda p \rho_{\alpha}^{\alpha}(\boldsymbol{z})\left(p b_{\nu}^{\nu} \gamma_{\delta}^{\delta}(\boldsymbol{z})+2 b_{\delta}^{\nu} \gamma_{\nu}^{\delta}(\boldsymbol{z})\right) \\
& \left.-2 \lambda x_{3}^{2} p \gamma_{\alpha}^{\alpha}(\boldsymbol{z})\left(P_{1}\right)_{\nu}^{\nu}(\boldsymbol{z})+2 \lambda x_{3}^{4} p \rho_{\alpha}^{\alpha}(\boldsymbol{z})\left(P_{2}\right)_{\nu}^{\nu}(\boldsymbol{z})+\text { h.o.t. }\right) \mathrm{d} t^{1} \mathrm{~d} t^{2} \mathrm{~d} t^{3} .
\end{aligned}
$$

Hence using (A.8) we see that $Q_{1}(\varepsilon, \boldsymbol{z})$ satisfies:

$$
\left|Q_{1}(\varepsilon, \boldsymbol{z})\right| \leq A E\left(\frac{\varepsilon^{2}}{R}|\boldsymbol{\gamma}|_{0 ; S}^{2}+\frac{\varepsilon^{4}}{R}|\boldsymbol{\rho}|_{0 ; S}^{2}+\frac{\varepsilon^{3}}{R}|\boldsymbol{\gamma}|_{0 ; S}|\boldsymbol{\rho}|_{0 ; S}\right)
$$


where we used the fact that $\varepsilon R^{-1}<1$. As we have

$$
\frac{\varepsilon^{3}}{R}|\gamma|_{0 ; S}|\rho|_{0 ; S} \leq A\left(\frac{\varepsilon^{2}}{R}|\gamma|_{0 ; S}^{2}+\frac{\varepsilon^{4}}{R}|\rho|_{0 ; S}^{2}\right)
$$

we get using (5.8)

$$
\left|Q_{1}(\varepsilon, \boldsymbol{z})\right| \leq A \frac{\varepsilon}{R} \mathrm{E}_{2 \mathrm{D}}^{\varepsilon}[\boldsymbol{z}]
$$

Similarly we compute that:

$$
\int_{\Omega^{\varepsilon}} \lambda \tilde{e}_{\alpha}^{\alpha}(\mathbf{W} \boldsymbol{z}) \tilde{e}_{\beta}^{\beta}(\mathbf{W} \boldsymbol{z}) \mathrm{d} t^{1} \mathrm{~d} t^{1} \mathrm{~d} t^{3}=2 \varepsilon \lambda \int_{S}\left[\gamma_{\alpha}^{\alpha}(\boldsymbol{z}) \gamma_{\beta}^{\beta}(\boldsymbol{z})+\frac{\varepsilon^{2}}{3} \rho_{\alpha}^{\alpha}(\boldsymbol{z}) \rho_{\beta}^{\beta}(\boldsymbol{z})\right] \mathrm{d} S+Q_{2}(\varepsilon, \boldsymbol{z})
$$

where, again, we have

$$
\left|Q_{2}(\varepsilon, \boldsymbol{z})\right| \leq A \frac{\varepsilon}{R} \mathrm{E}_{2 \mathrm{D}}^{\varepsilon}[\boldsymbol{z}]
$$

We also have

$$
\int_{\Omega^{\varepsilon}} 4 \mu a^{\alpha \beta}\left(x_{3}\right) \tilde{e}_{\alpha}^{3}(\mathbf{W} \boldsymbol{z}) \tilde{e}_{\beta}^{3}(\mathbf{W} \boldsymbol{z}) \mathrm{d} t^{1} \mathrm{~d} t^{1} \mathrm{~d} t^{3}=Q_{3}(\varepsilon, \boldsymbol{z})
$$

with:

$$
\left|Q_{3}(\varepsilon, \boldsymbol{z})\right| \leq A E\left(\varepsilon^{3}|\gamma|_{1 ; S}^{2}+\varepsilon^{5}|\boldsymbol{\rho}|_{1 ; S}^{2}\right)
$$

and thus using the definition (2.17) of $L_{1}$ and the estimates (5.8),

$$
\left|Q_{3}(\varepsilon, \boldsymbol{z})\right| \leq A E \frac{\varepsilon^{2}}{L_{1}^{2}} \mathrm{E}_{2 \mathrm{D}}^{\varepsilon}[\boldsymbol{z}]
$$

Finally, we have:

$$
\begin{aligned}
\int_{\Omega^{\varepsilon}} 2 \mu \tilde{e}_{\beta}^{\alpha}(\mathbf{W} \boldsymbol{z}) \tilde{e}_{\alpha}^{\beta}(\mathbf{W} \boldsymbol{z}) \mathrm{d} t^{1} \mathrm{~d} t^{1} \mathrm{~d} t^{3} & = \\
4 \varepsilon \mu \int_{S}\left[\gamma_{\beta}^{\alpha}(\boldsymbol{z}) \gamma_{\alpha}^{\beta}(\boldsymbol{z})+\frac{\varepsilon^{2}}{3} \rho_{\beta}^{\alpha}(\boldsymbol{z}) \rho_{\alpha}^{\beta}(\boldsymbol{z})\right] \mathrm{d} S+Q_{4}(\varepsilon, \boldsymbol{z}) &
\end{aligned}
$$

where, again:

$$
\left|Q_{4}(\varepsilon, \boldsymbol{z})\right| \leq A \frac{\varepsilon}{R} \mathrm{E}_{2 \mathrm{D}}^{\varepsilon}[\boldsymbol{z}] .
$$

Finally, using the relation: $\lambda-2 \lambda p+p^{2}(\lambda+2 \mu)=2 \mu p$, we find that

$$
\begin{aligned}
\tilde{E}_{3 \mathrm{D}}^{\varepsilon}[\mathbf{W} \boldsymbol{z}]=2 \varepsilon \int_{S}\left[2 \mu p \gamma_{\alpha}^{\alpha}(\boldsymbol{z}) \gamma_{\beta}^{\beta}(\boldsymbol{z})+2 \mu \gamma_{\alpha}^{\beta}(\boldsymbol{z}) \gamma_{\beta}^{\alpha}(\boldsymbol{z})\right] \mathrm{d} S \\
+\frac{2 \varepsilon^{3}}{3} \int_{S}\left[2 \mu p \rho_{\alpha}^{\alpha}(\boldsymbol{z}) \rho_{\beta}^{\beta}(\boldsymbol{z})+2 \mu \rho_{\alpha}^{\beta}(\boldsymbol{z}) \rho_{\beta}^{\alpha}(\boldsymbol{z})\right] \mathrm{d} S+Q(\varepsilon, \boldsymbol{z})
\end{aligned}
$$

where $Q(\varepsilon, \boldsymbol{z})$ is the sum $\sum_{\ell=1}^{4} Q_{\ell}(\varepsilon, \boldsymbol{z})$, and thus

$$
|Q(\varepsilon, \boldsymbol{z})| \leq A\left(\frac{\varepsilon}{R}+\frac{\varepsilon^{2}}{L_{1}^{2}}\right) \mathrm{E}_{2 \mathrm{D}}^{\varepsilon}[\boldsymbol{z}]
$$


But, compared with (A.5), the right-hand side of (A.9) writes $\mathrm{E}_{2 \mathrm{D}}^{\varepsilon}[\boldsymbol{z}]+Q(\varepsilon, \boldsymbol{z})$. Hence we have

$$
\tilde{\mathrm{E}}_{3 \mathrm{D}}^{\varepsilon}[\mathbf{W} \boldsymbol{z}]-\mathrm{E}_{2 \mathrm{D}}^{\varepsilon}[\boldsymbol{z}]=Q(\varepsilon, \boldsymbol{z})
$$

and this yields the result.

Remark A.3 The part $U^{\mathrm{cmp}} \boldsymbol{z}$ has a significant energy. If we evaluate the energy of $\mathrm{U}^{\mathrm{KL}} \boldsymbol{z}$ instead of the full $\mathrm{U} \boldsymbol{z}$, we obtain the plain strain energy $2 \varepsilon b_{2 \mathrm{D}}^{\varepsilon}(\boldsymbol{z}, \boldsymbol{z})$ of $\boldsymbol{z}$ defined below instead of the plain stress energy $2 \varepsilon a_{2 \mathrm{D}}^{\varepsilon}(\boldsymbol{z}, \boldsymbol{z})$ : Recall that, cf. (2.9) $a_{2 \mathrm{D}}^{\varepsilon}(\boldsymbol{z}, \boldsymbol{z})$ is equal to

$$
\int_{S} \tilde{\lambda} \gamma_{\alpha}^{\alpha}(\boldsymbol{z}) \gamma_{\beta}^{\beta}(\boldsymbol{z})+2 \mu \gamma_{\alpha}^{\beta}(\boldsymbol{z}) \gamma_{\beta}^{\alpha}(\boldsymbol{z}) \mathrm{d} S+\frac{\varepsilon^{2}}{3} \int_{S} \tilde{\lambda} \rho_{\alpha}^{\alpha}(\boldsymbol{z}) \rho_{\beta}^{\beta}(\boldsymbol{z})+2 \mu \rho_{\alpha}^{\beta}(\boldsymbol{z}) \rho_{\beta}^{\alpha}(\boldsymbol{z}) \mathrm{d} S
$$

and let us define $b_{2 \mathrm{D}}^{\varepsilon}(\boldsymbol{z}, \boldsymbol{z})$ as

$$
\int_{S} \lambda \gamma_{\alpha}^{\alpha}(\boldsymbol{z}) \gamma_{\beta}^{\beta}(\boldsymbol{z})+2 \mu \gamma_{\alpha}^{\beta}(\boldsymbol{z}) \gamma_{\beta}^{\alpha}(\boldsymbol{z}) \mathrm{d} S+\frac{\varepsilon^{2}}{3} \int_{S} \lambda \rho_{\alpha}^{\alpha}(\boldsymbol{z}) \rho_{\beta}^{\beta}(\boldsymbol{z})+2 \mu \rho_{\alpha}^{\beta}(\boldsymbol{z}) \rho_{\beta}^{\alpha}(\boldsymbol{z}) \mathrm{d} S .
$$

Using the previous computations, we can show that

$$
\left|\mathrm{E}_{3 \mathrm{D}}^{\varepsilon}\left[\mathrm{U}^{\mathrm{KL}} \boldsymbol{z}\right]-2 \varepsilon b_{2 \mathrm{D}}^{\varepsilon}(\boldsymbol{z}, \boldsymbol{z})\right| \leq A E\left(\frac{\varepsilon^{2}}{R}|\boldsymbol{\gamma}|_{0 ; S}^{2}+\frac{\varepsilon^{4}}{R}|\boldsymbol{\rho}|_{0 ; S}^{2}\right) .
$$

\section{References}

[1] S. Agmon, A. Douglis, L. Nirenberg. Estimates near the boundary for solutions of elliptic partial differential equations satisfying general boundary conditions II. Comm. Pure Appl. Math. 17 (1964) 35-92.

[2] O. Alexandrescu. Théorème d'existence pour le modèle bidimensionnel de coque non linéaire de w. t. koiter. C R. Acad. Sci. Paris, Sér. I. (1994) 899-902.

[3] G. Andreoiu, M. Dauge, E. Faou. Développements asymptotiques complets pour des coques faiblement courbées encastrées ou libres. C. R. Acad. Sci. Paris Sér. I Math. 330(6) (2000) 523-528.

[4] G. Andreoiu, E. FAOU. Asymptotics of arbitrary order for clamped and free shallow shells. To appear in Asympt. Anal. (2001).

[5] D. N. Arnold, A. L. Madureira, S. Zhang. On the range of applicability of the Reissner-Mindlin and Kirchhoff-Love plate bending models. J. Elasticity 67 (2002) 171-185 (2003).

[6] M. Bernadou, P. G. Ciarlet. Sur l'ellipticité du modèle linéaire de coques de W.T.Koiter. In R.Glowinski, J.L.Lions, editors, Computing Methods in Applied Sciences and Engineering, Lecture Notes in Economics and Mathematical Systems, Vol.134, pages 89-136. Springer-Verlag, Heidelberg 1976.

[7] A. Blouza, H. Le Dret. Existence and uniqueness for the linear Koiter model for shells with little regularity. Quart. Appl. Math. 57 (1999) 317-337. 
[8] B. Budiansky, J. L. Sanders. On the "best" first-order linear shell theory. In $W$. Prager Anniversary Volume, Progress in Applied Mechanics, pages 129-140. Macmillan, New-York 1967.

[9] S. Busse, P. G. Ciarlet, B. Miara. Justification d'un modele lineaire bidimensionnel de coques "faiblement courbees" en coordonnees curvilignes. RAIRO Modél. Math. Anal. Numer. 31 (3) (1997) 409-434.

[10] P. G. Ciarlet, V. Lods. Asymptotic analysis of linearly elastic shells. I. Justification of membrane shell equations. Arch. Rational Mech. Anal. 136 (1996) 119-161.

[11] P. G. Ciarlet, V. Lods. Asymptotic analysis of linearly elastic shells. III. Justification of Koiter's shell equations. Arch. Rational Mech. Anal. 136 (1996) 191-200.

[12] P. G. Ciarlet, V. Lods, B. Miara. Asymptotic analysis of linearly elastic shells. II. Justification of flexural shell equations. Arch. Rational Mech. Anal. 136 (1996) 163-190.

[13] P. G. Ciarlet, J. C. Paumier. A justification of the marguerre-von- kármán equations. Computational Mechanics 1 (1986) 177-202.

[14] M. Dauge, I. Djurdjevic, A. Rössle. Higher order bending and membrane responses of thin linearly elastic plates. C. R. Acad. Sci. Paris, Sér. I, 326 (1998) 519524.

[15] M. Dauge, I. Gruais. Asymptotics of arbitrary order for a thin elastic clamped plate. I: Optimal error estimates. Asymptotic Analysis 13 (1996) 167-197.

[16] M. Dauge, I. Gruais, A. Rössle. The influence of lateral boundary conditions on the asymptotics in thin elastic plates. SIAM J. Math. Anal. 31(2) (1999/00) 305-345 (electronic).

[17] M. P. Do CARMo. Riemannian geometry. Mathematics : Theory and applications. Birkhäuser, Boston 1992.

[18] E. FAOU. Développements asymptotiques dans les coques linéairement élastiques. Thèse, Université de Rennes 1 (2000).

[19] E. FAOU. Elasticity on a thin shell: Formal series solution. Asymptotic Analysis 31 (2002) 317-361.

[20] E. FAOU. Multiscale expansions for linear clamped elliptic shells. Communications in Partial Differential Equations 29(11 \& 12) (2004) 1799-1845.

[21] A. L. Gol'denveizer. Theory of Elastic Thin Shells. Pergamon, New York 1961.

[22] H. Hakula. Propagation of singularities on thin shells with hyperbolic regions. In Numerical mathematics and advanced applications, pages 357-363. Springer Italia, Milan 2003.

[23] F. John. Refined interior equations for thin elastic shells. Comm. Pure Appl. Math. 24 (1971) 583-615.

[24] G. KIRCHHOFF. Über das Gleichgewicht und die Bewegung einer elastischen Scheibe. Journ. Reine Angew. Math. 40 (1850) 51-58. 
[25] W. T. Koiter. A consistent first approximation in the general theory of thin elastic shells. Proc. IUTAM Symposium on the Theory on Thin Elastic Shells, August 1959 (1960) 12-32.

[26] W. T. Koiter. On the foundations of the linear theory of thin elastic shells: I. Proc. Kon. Ned. Akad. Wetensch., Ser.B 73 (1970) 169-182.

[27] W. T. Koiter. On the foundations of the linear theory of thin elastic shells: II. Proc. Kon. Ned. Akad. Wetensch., Ser.B 73 (1970) 183-195.

[28] W. T. Koiter, J. C. Simmonds. Foundations of shell theory. In E. Becker, G. K. Mikhailov, editors, Applied Mechanics, Proceedings of the Thirteenth International Congress of Theoretical and Applied Mechanics, pages 150-176. SpringerVerlag, Berlin 1973.

[29] V. Lods, C. Mardare. Asymptotic justification of the Kirchhoff-love hypotheses for a linearly elastic clamped shell. J. Elasticity 58 (2000) 105-154.

[30] V. LODS, C. MARDARE. Justification of linear Koiter and Naghdi's models for totally clamped shell. Asymptotic Anal. 31 (2002) 189-210.

[31] V. G. Maz'ya, S. A. Nazarov, B. A. PlamenevskiI. Asymptotische Theorie elliptischer Randwertaufgaben in singulär gestörten Gebieten II. Mathematische Monographien, Band 83. Akademie Verlag, Berlin 1991.

[32] P. M. NAGHdi. Foundations of elastic shell theory. In Progress in Solid Mechanics, volume 4, pages 1-90. North-Holland, Amsterdam 1963.

[33] S. A. NAZARov, I. S. Zorin. Edge effect in the bending of a thin three-dimensional plate. Prikl. Matem. Mekhan. 53 (4) (1989) 642-650. English translation J. Appl. Maths. Mechs. (1989) 500-507.

[34] A. H. Niemi, J. Pitkëranta, H. Hakula. Benchmark computations on pointloaded shallow shells: Fourier vs. FEM. Comput. Methods Appl. Mech. Engrg. 196(46) (2007) 894-907.

[35] V. V. Novozhilov. Thin Shell Theory. Walters-Noordhoff Publishing, Groningen 1959.

[36] J. Pitkäranta, A.-M. Matache, C. Schwab. Fourier mode analysis of layers in shallow shell deformations. Comput. Methods Appl. Mech. Eng. 190 (2001) 29432975.

[37] E. Sanchez-PALEncia. Passage à la limite de l'élasticité tridimensionnelle à la théorie asymptotique des coques minces. C. R. Acad. Sci. Paris, Sér. II 311 (1990) 909-916.

[38] M. SPIVAK. A comprehensive introduction to differential geometry. Publish or perish 1979. 